ORNL/TM-13334, R2

\title{
MCNP-DSP USERS MANUAL
}

J anuary 2001

TIMOTHY E. VALENTINE 


\title{
DOCUMENT AVAILABILITY
}

Reports produced after January 1, 1996, are generally available free via the U.S. Department of Energy (DOE) Information Bridge:

Web site: http://www.osti.gov/bridge

Reports produced before January 1, 1996, may be purchased by members of the public from the following source:

\author{
National Technical Information Service \\ 5285 Port Royal Road \\ Springfield, VA 22161 \\ Telephone: 703-605-6000 (1-800-553-6847) \\ TDD: 703-487-4639 \\ Fax: 703-605-6900 \\ E-mail: info@ntis.fedworld.gov \\ Web site: http://www.ntis.gov/support/ordernowabout.htm
}

Reports are available to DOE employees, DOE contractors, Energy Technology Data Exchange (ETDE) representatives, and International Nuclear Information System (INIS) representatives from the following source:

Office of Scientific and Technical Information

P.O. Box 62

Oak Ridge, TN 37831

Telephone: 865-576-8401

Fax: 865-576-5728

E-mail: reports@adonis.osti.gov

Web site: http://www.osti.gov/contact.html

This report was prepared as an account of work sponsored by an agency of the United States Government. Neither the United States government nor any agency thereof, nor any of their employees, makes any warranty, express or implied, or assumes any legal liability or responsibility for the accuracy, completeness, or usefulness of any information, apparatus, product, or process disclosed, or represents that its use would not infringe privately owned rights. Reference herein to any specific commercial product, process, or service by trade name, trademark, manufacturer, or otherwise, does not necessarily constitute or imply its endorsement, recommendation, or favoring by the United States Government or any agency thereof. The views and opinions of authors expressed herein do not necessarily state or reflect those of the United States Government or any agency thereof. 
ORNL/TM-13334, R2

Computational Physics and Engineering Division

\title{
MCNP-DSP Users Manual
}

\author{
Timothy E. Valentine
}

January 2001

\author{
Prepared by the \\ OAK RIDGE NATIONAL LABORATORY \\ P.O. Box 2008 \\ Oak Ridge, Tennessee 37831-6285 \\ managed by \\ UT-BATTELLE, LLC \\ for the \\ U.S. DEPARTMENT OF ENERGY \\ under contract DE-AC05-00OR22725
}




\section{Table of Contents}

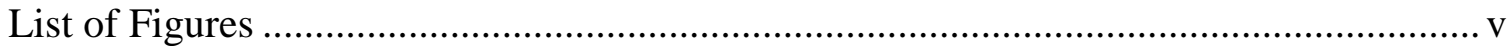

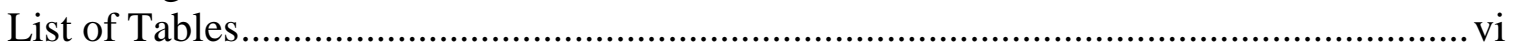

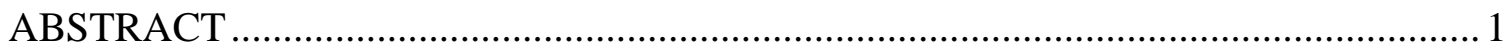

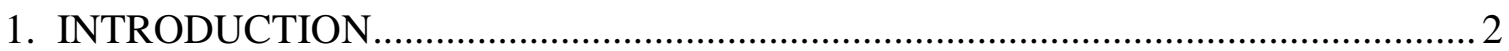

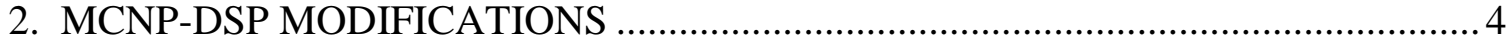

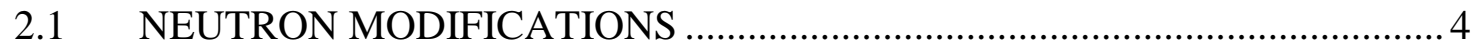

2.1.1 Number of Prompt Neutrons from Fission............................................ 4

2.1.2 Angular Distribution of Prompt Neutrons from Fission ............................ 6

2.1.3 Fission Neutron Energy Distribution ............................................ 8

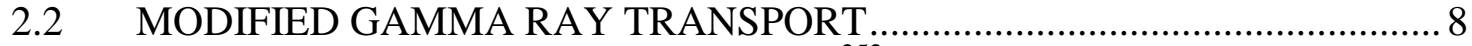

2.2.1 Prompt Gamma Ray Multiplicity for ${ }^{252} \mathrm{Cf}$.......................................... 9

2.2.2 Prompt Gamma Ray Energy Distribution for ${ }^{252} \mathrm{Cf}$............................... 9

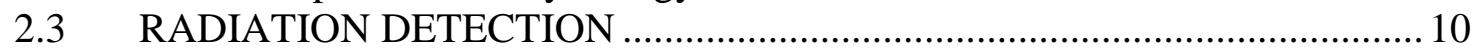

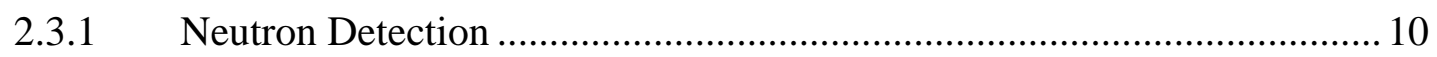

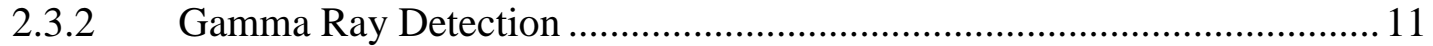

2.3.3 Dead Time Models ........................................................................... 11

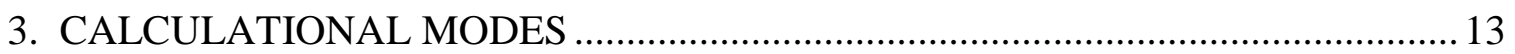

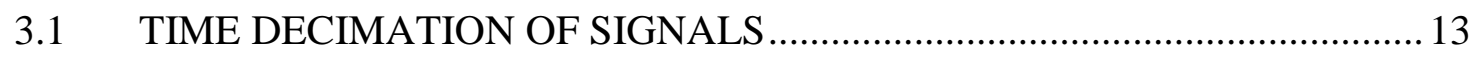

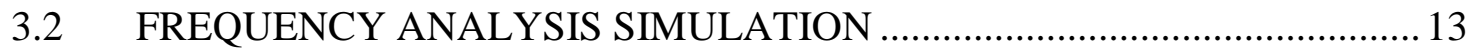

3.2.1 Source-Driven Noise Analysis Simulations ........................................... 16

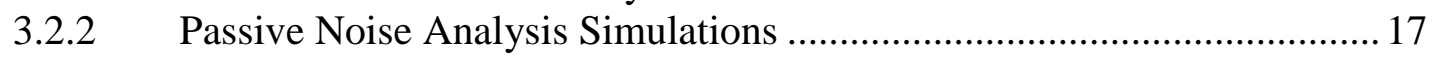

3.3 CORRELATION ANALYSIS SIMULATION .......................................... 17

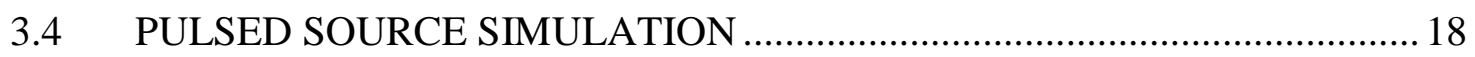

3.5 FEYNMAN VARIANCE SIMULATION ............................................. 19

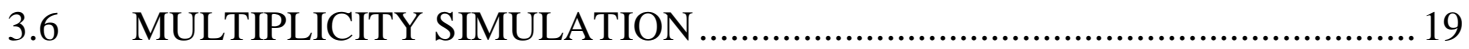

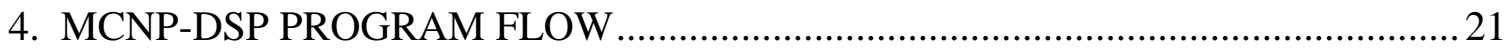

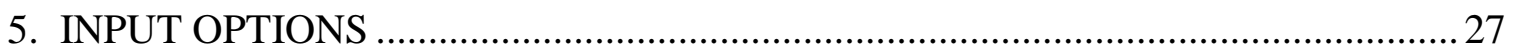

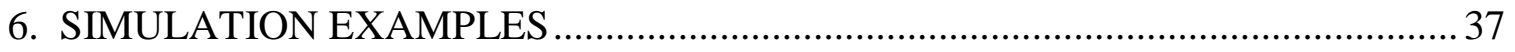

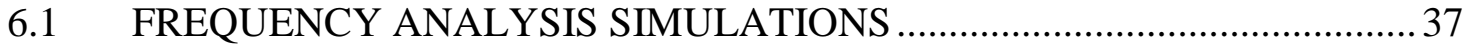

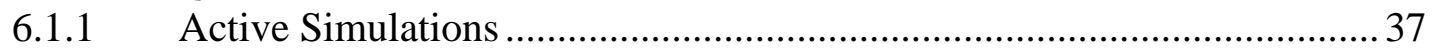

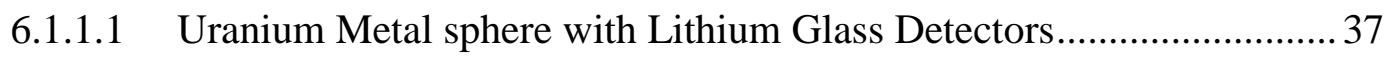

6.1.1.2 Uranium Metal Sphere with Plastic Scintillation Detectors................. 45

6.1.1.3 Uranium Metal Sphere with Composite Detectors ............................. 48

6.1.1.4 Uranyl Nitrate Solution with ${ }^{3}$ He Detectors.......................................5 52

6.1.1.5 Uranium Metal Sphere with Plastic Scintillation Detectors and

Continuous, Isotropic D-T Source ................................................................ 57

6.1.1.6 Uranium Metal Spheres with Plastic Scintillation Detectors and Pulsed,

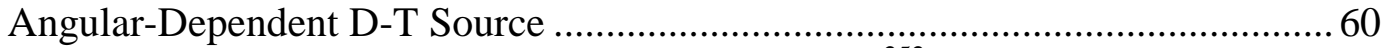

6.1.1.7 Delta Phase Plutonium Metal Sphere with at ${ }^{252} \mathrm{Cf}$ Source......................6 62

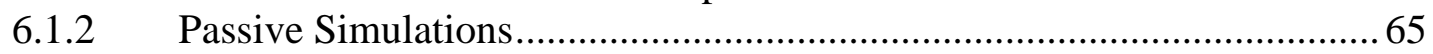

6.1.2.1 Nested Plutonium Shells with Plastic Scintillation Detectors...............65

6.1.2.2 Adjacent Plutonium Cylinders with Plastic Scintillation Detectors......69

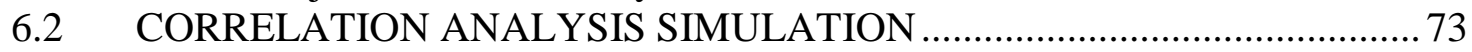

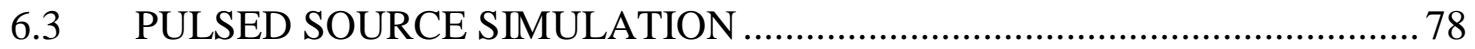


6.4 FEYNMAN VARIANCE SIMULATION ............................................... 83

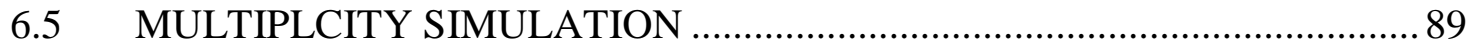

APPENDIX A MCNP-DSP INPUT AND OUTPUT FILES …..................................95

APPENDIX B SPONTANEOUS FISSION NEUTRON DISTRIBUTIONS .................99

APPENDIX C SPONTANEOUS FISSION NEUTRON ENERGY SPECTRA ........... 102

APPENDIX D SPONTANEOUS FISSION GAMMA RAY DISTRIBUTIONS .......... 105

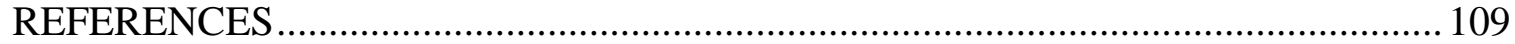




\section{List of Figures}

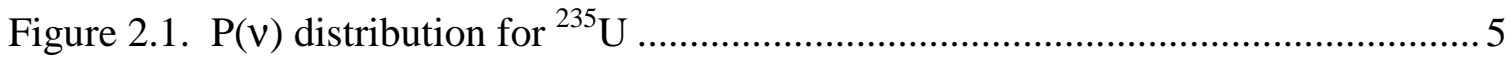

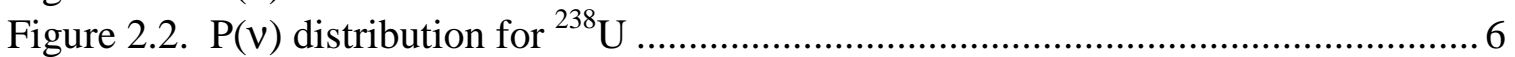

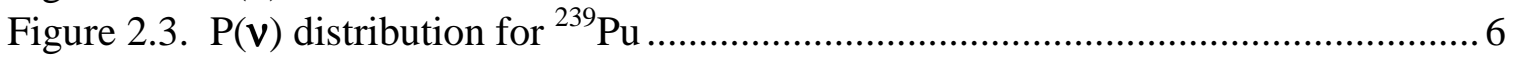

Figure 2.4. Probability density function for angular distribution (lab system) of neutrons

from fission with respect to the direction of the light fragment ................................ 7

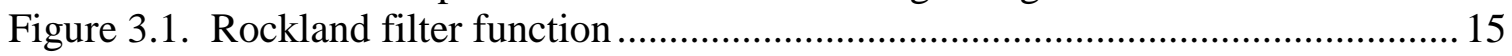

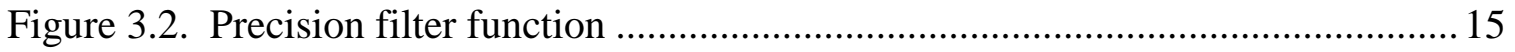

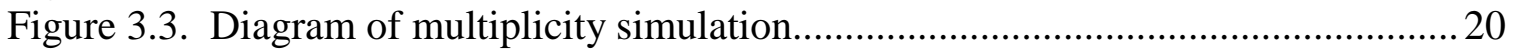

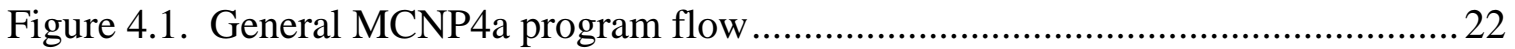

Figure 4.2. Structure of inner and outer loops of MCNP-DSP ..................................... 24

Figure 4.3. Particle tracking procedure ....................................................................... 25

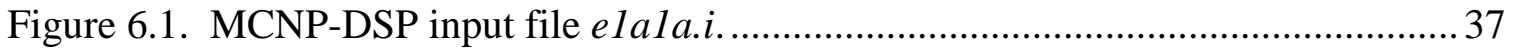

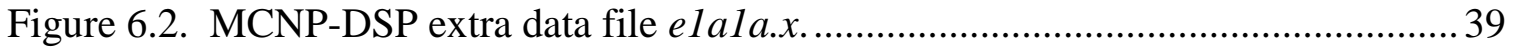

Figure 6.3. MCNP-DSP output summary file e lala.1 .............................................. 41

Figure 6.4. MCNP-DSP output file elala.1.123.fft.n ................................................. 44

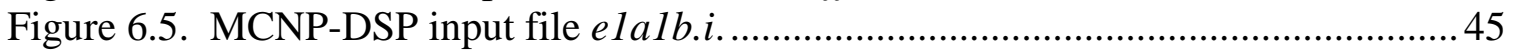

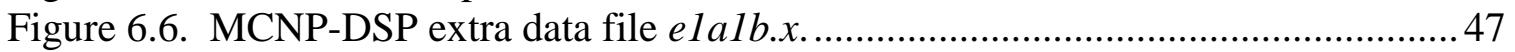

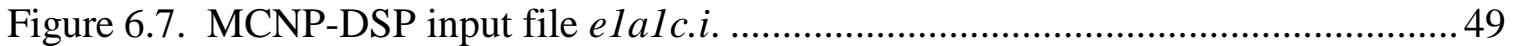

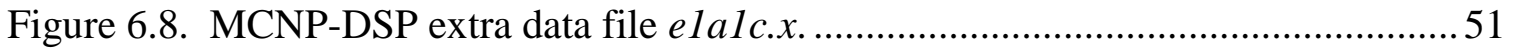

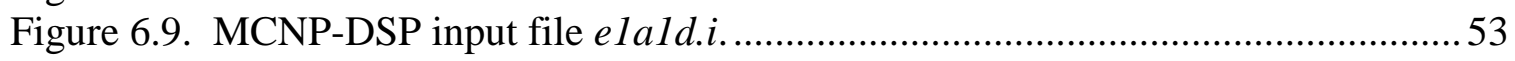

Figure 6.10. MCNP-DSP extra data file elald.x......................................................56

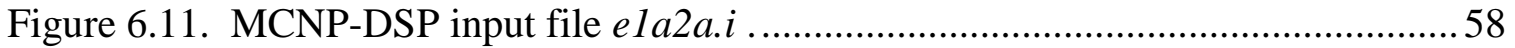

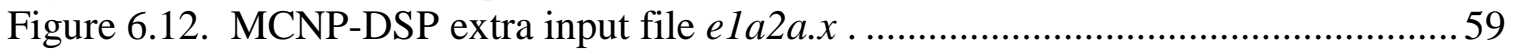

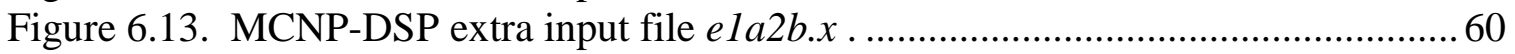

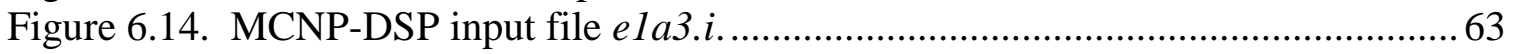

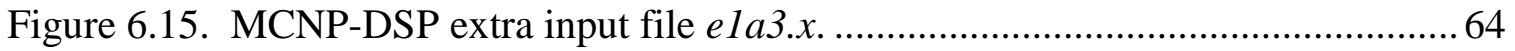

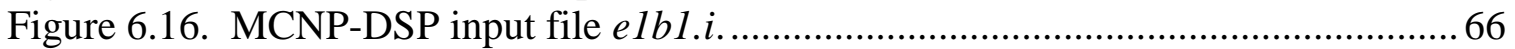

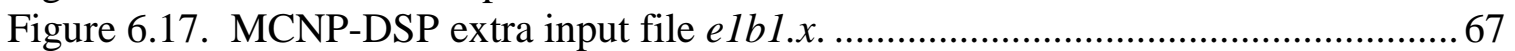

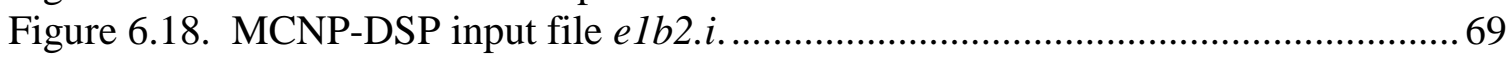

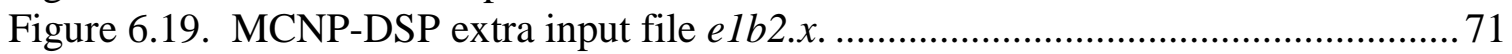

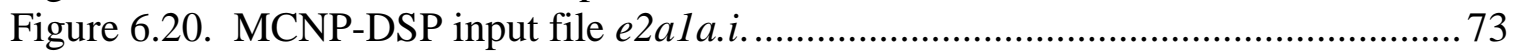

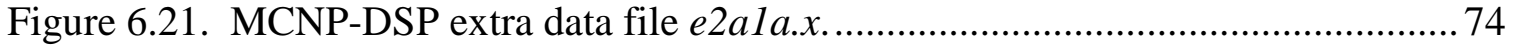

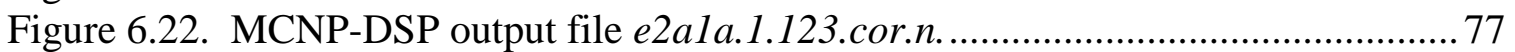

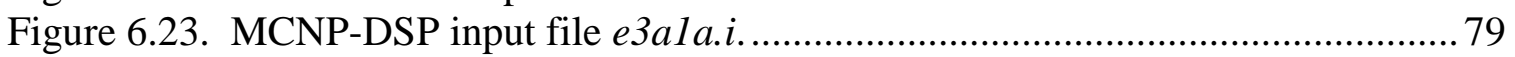

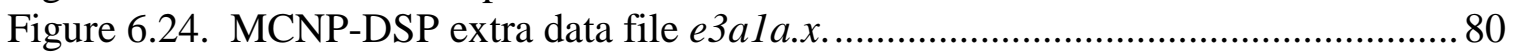

Figure 6.25. MCNP-DSP pulsed source output file e3ala.1.tim.................................. 82

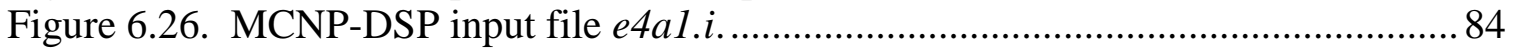

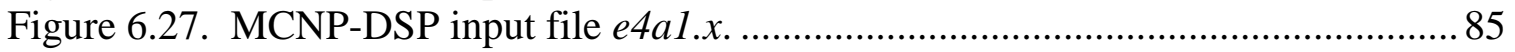

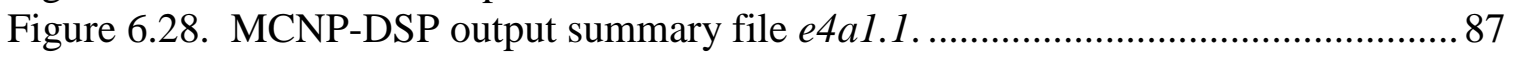

Figure 6.29. MCNP-DSP input file e5ala.i........................................................... 90

Figure 6.30. MCNP-DSP extra data file e5ala.x.................................................... 91

Figure 6.31. MCNP-DSP multiplicity output file e 5 ala.1.mlt ..................................... 94 


\section{List of Tables}

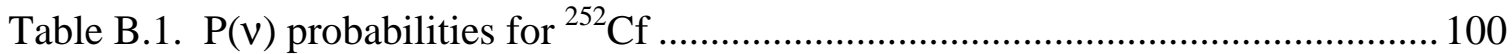

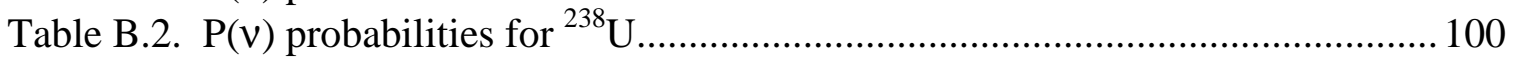

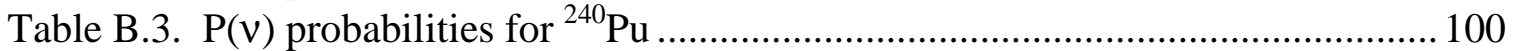

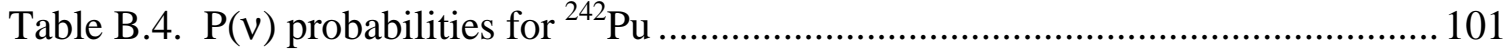

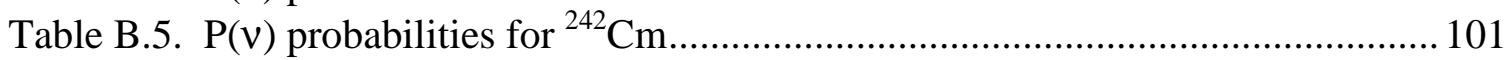

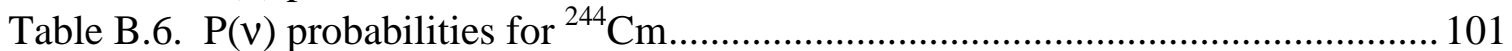

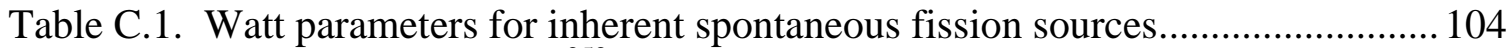

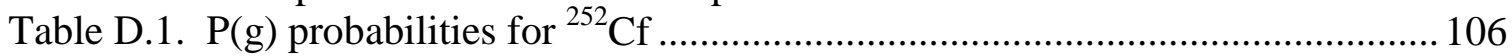

Table D.2. Parameters of gamma rays from spontaneous fission ................................ 107 


\begin{abstract}
The Monte Carlo code MCNP-DSP was developed from the Los Alamos MCNP4a code to calculate the time and frequency response statistics obtained from subcritical measurements. The code can be used to simulate a variety of subcritical measurements including source-driven noise analysis, Rossi- $\alpha$, pulsed source, passive frequency analysis, multiplicity, and Feynman variance measurements. This code can be used to validate Monte Carlo methods and cross section data sets with subcritical measurements and replaces the use of point kinetics models for interpreting subcritical measurements.
\end{abstract}




\section{INTRODUCTION}

The Monte Carlo code MCNP-DSP ${ }^{1,2}$ was developed from the MCNP4a ${ }^{3, T M}$ code to calculate the time and frequency measured parameters of the ${ }^{252} \mathrm{Cf}$-source-driven noise analysis method. ${ }^{4}$ This measurement technique was developed to determine the subcriticality of fissile assemblies. In the past, point reactor kinetics expressions were used to relate the measured spectra to the subcritical neutron multiplication for criticality safety applications. However, this limited the applications of this measurement technique because point reactor kinetics models are not applicable to all measurement configurations.

The KENO-NR ${ }^{5}$ Monte Carlo code was developed by Ficaro from KENO V. $\mathrm{a}^{6}$ to calculate the spectral densities of this measurement. However, this code is limited in that it only calculates the detector responses due to neutrons and uses group cross section, whereas the most efficient detectors used in the measurements detect both neutrons and gamma rays. Sometimes gamma ray detectors are used exclusively in the measurements. Because of these limitations, a more generalized Monte Carlo model that uses continuous energy cross-sections and also includes gamma rays was needed to calculate the spectral densities.

The Monte Carlo code MCNP4a was modified to calculate both the time and frequency domain signatures from source-driven subcritical measurements to provide a more detailed model for interpretation of the measured results. Several modifications were made to the neutron and gamma ray tracking routines of MCNP such that the calculated parameters are obtained as measured. The structure of the code was modified to obtain data blocks of detector responses, as in measurements. The data blocks are time samples of detector response, typically of 512 or 1024 points, for a certain period, which is determined from the sampling rate of the data acquisition system. The particle splitting and biasing in MCNP were disabled in order to have a strictly analog Monte Carlo calculation that follows actual particle random walks. A dual-particle source was developed which produces both neutrons and gamma rays from the fission of ${ }^{252} \mathrm{Cf}$ in which the time tracking of the gamma rays was coupled to that of the neutrons. Additional evaluated measured data were incorporated into the code for certain neutron and gamma ray interactions to more precisely follow the physics of particle interaction on an event-byevent basis. Average quantities like $\bar{v}$, the average number of prompt neutrons per fission, were removed from MCNP because average quantities remove some of the statistical fluctuations from the fission chain populations. Experimental data describing the probability distribution of the number of prompt neutrons from fission were incorporated into the code where available; otherwise, a Gaussian theoretical distribution is used. A modified Maxwellian energy distribution was included in the code to obtain the energy of prompt neutrons from the spontaneous fission of ${ }^{252} \mathrm{Cf}$ along with angular distribution data for neutrons from fission to describe anisotropy of the neutron directions in the laboratory system. The energy distribution of gamma rays from the spontaneous fission of ${ }^{252} \mathrm{Cf}$ and

\footnotetext{
${ }^{\mathrm{TM}}$ MCNP is a trademark of the Regents of the University of California, Los Alamos National Laboratory.
} 
the multiplicity of these gamma rays was also incorporated into MCNP-DSP. MCNP-DSP correlates both the neutrons and gamma rays in time throughout the fission chain multiplication process. However, as in MCNP, the gamma ray production is not directly correlated to the type of neutron event. Several detector options were included in MCNPDSP to allow calculation of the responses of the various neutron and gamma sensitive detectors used in the measurements. The detectors used in MCNP-DSP are capture, scatter, or fission detectors. A simplified algorithm for treatment of multiple scattering events in the scatter detectors was included in addition to multiple scattering of particles between detectors. Data processing algorithms were also incorporated into the code to process the detector responses for both time and frequency analysis. The frequency spectra are obtained by directly calculating the Fourier transforms of $N$ point blocks of pulses from the detectors and performing a complex multiplication to obtain the auto spectra and the cross spectra.

The modifications that were made to simulate the source-driven noise analysis measurement are also needed to simulate other subcritical measurements. The code is capable of simulating Rossi- $\alpha,{ }^{7}$ pulsed neutron, ${ }^{8}$ passive frequency analysis, ${ }^{9}$ multiplicity, ${ }^{10}$ and Feynman variance ${ }^{11}$ measurements. The user should consider using the standard MCNP program for Monte Carlo calculations that are not simulations of subcritical measurements.

This manual provides a description of the modifications made to the MCNP4a code and some general information about signal processing. The modifications to the neutron and photon transport processes are described and a brief explanation of the detection process is presented in Chap. 2. A description of the simulation of subcritical measurements is provided in Chap. 3. A short outline of the program flow is presented in Chap. 4. Descriptions of the input options for the code are provided in Chapter 5 while Chapter 6 contains examples for the various subcritical measurement simulations. Additional information is provided in the Appendices. Appendix A provides a description of the MCNP-DSP input and output files for the Unix and PC operating systems. The neutron emission distributions for spontaneous fission isotopes are given in Appendix B while the neutron energy spectra parameters for spontaneous fission isotopes are provided in Appendix C. The photon emission distributions for spontaneous fission nuclides are provided in Appendix D. Details concerning the use of MCNP are not included in this manual. It is assumed that the reader is familiar with the standard MCNP input. 


\section{MCNP-DSP MODIFICATIONS}

Although MCNP is very versatile, several changes were made to the code to accurately estimate the time-and frequency-analysis statistics. A dual-particle source was developed which produced both time-correlated neutrons and gamma rays. The variance reduction features were disabled in order to have strictly analog particle tracking, and the neutron and gamma ray transport routines were modified to better represent the collision physics on an event-by-event basis.

\section{$2.1 \quad$ NEUTRON MODIFICATIONS}

Because many of the parameters obtained from the subcritical measurements depends on the fluctuations of the individual fission chains in a fissile configuration, it is necessary that the calculation be able to adequately simulate the behavior of the individual fission chains. This requires replacing average parameters such as $\bar{v}$ with probability distributions because the measured quantities are estimates of the fission chain fluctuations and because the average quantities reduce the fission chain fluctuations. The proper treatment of the angular distribution of neutrons from fission was also included in the calculation to adequately describe the particle direction from fission for both spontaneous fission and induced fission. The selection of the energy of the neutrons from the spontaneous fission of ${ }^{252} \mathrm{Cf}$ has been modified to incorporate recent improvements in the representation of the prompt neutron energy spectrum. Implementing these changes along with analog neutron tracking allow for the more physical representation of the individual fission chains.

\subsubsection{Number of Prompt Neutrons from Fission}

The probability of obtaining prompt neutrons from fission, $P(v)$, for various fission isotopes was functionally described by Terrell. ${ }^{12}$ Terrell suggested that the neutron multiplicities are dependent on the fission fragment properties. If a Gaussian distribution of the fission fragment excitation energies is assumed, the cumulative probability of observing $v$ prompt neutrons from fission is approximated as

$$
\sum_{n=0}^{v} P(n)=\frac{1}{\sqrt{2 \pi}} e_{-\infty}^{(v-\bar{v}-1 / 2+b) / \sigma} e^{\frac{-1}{2} t} d t,
$$

where $b$ is a small correction factor $\left(<10^{-2}\right)$ to ensure that the $v^{\prime}$ s are positive, and $\sigma$ is the root-mean-square width of the initial total excitation-energy distribution $(\sigma=1.08) .{ }^{12}$ Terrell has shown that this distribution function can be used to describe the emission probability for several different fission isotopes. In MCNP, the average number of neutrons from fission obtained from the cross-section data sets is a function of the energy of the neutron that induces the fission. Thus, the emission probability that was 
incorporated into MCNP-DSP is an energy dependent distribution. The Gaussian distribution is an approximation to describe the prompt neutron emission probabilities at all energies and is used unless additional measured distribution data tables are included for certain isotopes.

An option has been incorporated into MCNP-DSP to use the measured data summarized by Zucker and Holden. ${ }^{13}$ Zucker and Holden tabulated the $P(v)$ distributions for ${ }^{235} \mathrm{U},{ }^{238} \mathrm{U}$, and ${ }^{239} \mathrm{Pu}$ as a function of the energy of the neutron that induces the fission. These distributions are presented in Figs. 2.1 to 2.3. A modified $P(v)$ distribution has been incorporated for ${ }^{235} \mathrm{U}$. The modified distribution uses the $P(v)$ distribution at thermal energies that was measured by Gwin, Spencer, and Ingle. ${ }^{14}$ In previous versions of the code, the probability distribution data were fit with least-square polynomials to obtain a functional representation of the energy dependence of neutron emission probabilities. The Zucker and Holden data for ${ }^{235} \mathrm{U},{ }^{238} \mathrm{U}$, and ${ }^{239} \mathrm{Pu}$ were also used to fit the $P(v)$ distribution as a function of the average number of neutrons from fission. These fits are based on the observations of Frehaut ${ }^{15}$ who performed such an analysis for fast neutron-induced fission. The fit of the data for ${ }^{235} \mathrm{U}$ is used for both ${ }^{235} \mathrm{U}$ and ${ }^{233} \mathrm{U}$ neutron induced fission. The fit of the data for ${ }^{238} \mathrm{U}$ is used for ${ }^{232} \mathrm{U},{ }^{234} \mathrm{U},{ }^{236} \mathrm{U}$, and ${ }^{238} \mathrm{U}$ neutron induced fission. The fit for ${ }^{239} \mathrm{Pu}$ is used for both ${ }^{239} \mathrm{Pu}$ and ${ }^{241} \mathrm{Pu}$. The probability distribution is determined by sampling the average number of neutrons from fission from the cross section data file and estimating the individual probabilities of zero, one, two, etc., neutrons from fission using the fits of the distribution. The probability distribution data for ${ }^{252} \mathrm{Cf}$ were obtained from Spencer's measurements. ${ }^{16}$ An option has also been included to use the Boldeman et. al. distribution for ${ }^{252} \mathrm{Cf}$ spontaneous fission. ${ }^{17}$ The probability distribution data for the inherent spontaneous fission sources was taken from Holden and Zucker. ${ }^{18}$ The distribution data for ${ }^{252} \mathrm{Cf}$ and the spontaneous fission isotopes are given in Appendix B.

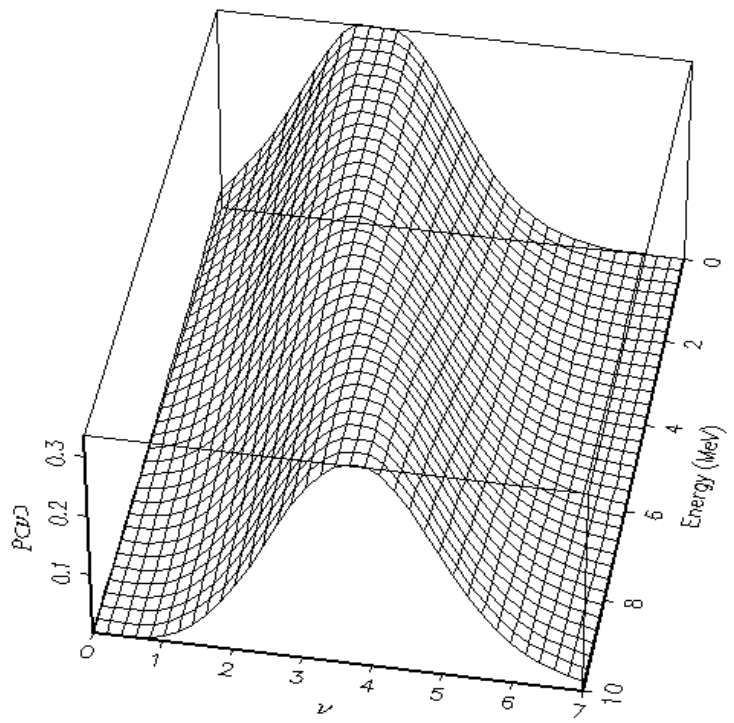

Figure 2.1. $P(v)$ distribution for ${ }^{235} U$ 


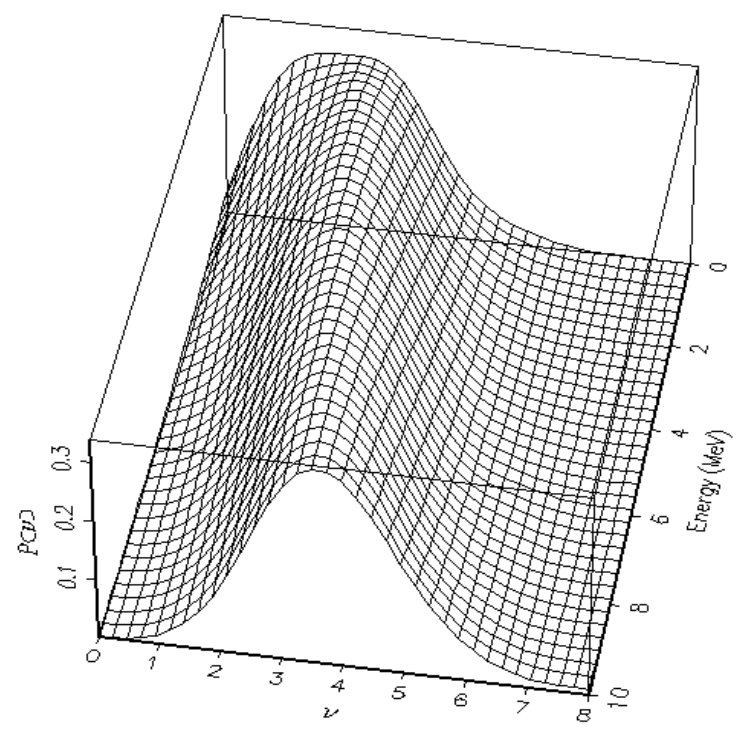

Figure 2.2. $\mathbf{P}(v)$ distribution for ${ }^{238} \mathbf{U}$

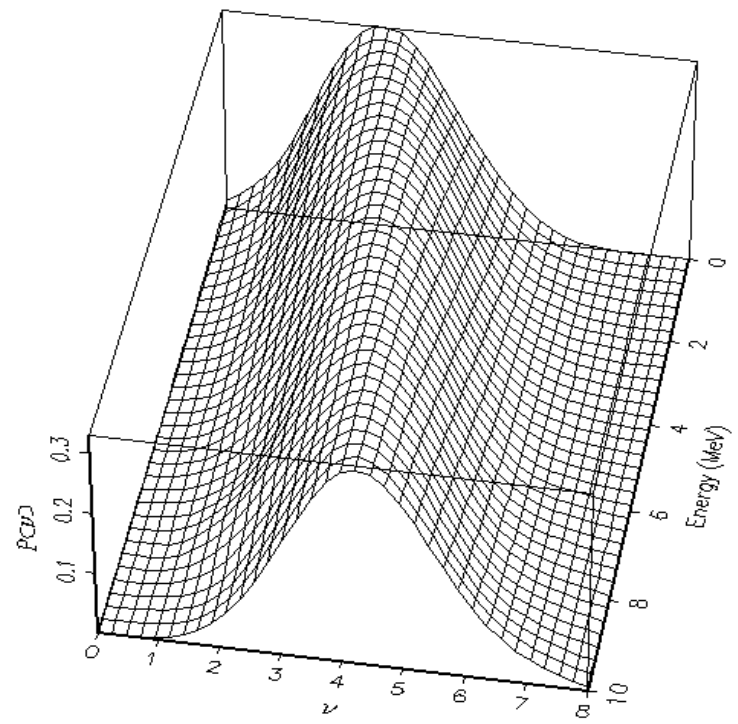

Figure 2.3. $P(v)$ distribution for ${ }^{239} \mathrm{Pu}$

\subsubsection{Angular Distribution of Prompt Neutrons from Fission}

There are instances in which the angular distributions of prompt neutrons from fission may be important, such as when the ${ }^{252} \mathrm{Cf}$ source is located some distance from the fissile configuration. Hence, assuming an isotropic emission of neutrons from fission can be a poor approximation. Budtz-Jorgensen and Knitter ${ }^{19}$ measured the angular distribution of neutrons relative to the direction of the light fission fragment for the spontaneous fission of ${ }^{252} \mathrm{Cf}$. Budtz-Jorgensen and Knitter have shown that there is angular anisotropy in the 
laboratory reference frame. The probability distribution function obtained from the BudtzJorgensen and Knitter data is shown in Fig. 2.4. As shown in Fig. 2.4, the angular distribution at zero energy is more isotropic; however, as the neutron energy increases, the angular distribution becomes very anisotropic.

These data are incorporated into MCNP-DSP by selecting the direction of the light fission fragment from an isotropic distribution. The neutron direction is then determined by sampling its azimuthal direction uniformly on the interval 0 to $2 \pi$. The polar angle of each fission neutron relative to the light fission fragment is determined from the angular probability distribution function. These data may be used for both the spontaneous fission of the ${ }^{252} \mathrm{Cf}$ source and for induced fission events in the system to investigate the effects of the angular dependence of the neutron emission. Since the majority of the neutrons are released from the fully accelerated fission fragments, the angular distribution of the neutrons relative to the direction of the fission fragments is dependent on the de-excitation of the fission fragments. Therefore, there would be little difference between the angular distribution of spontaneous fission neutrons and the angular distribution of induced fission neutrons for fissile isotopes. For these reasons the data for ${ }^{252} \mathrm{Cf}$ were used for all fission. ${ }^{20}$

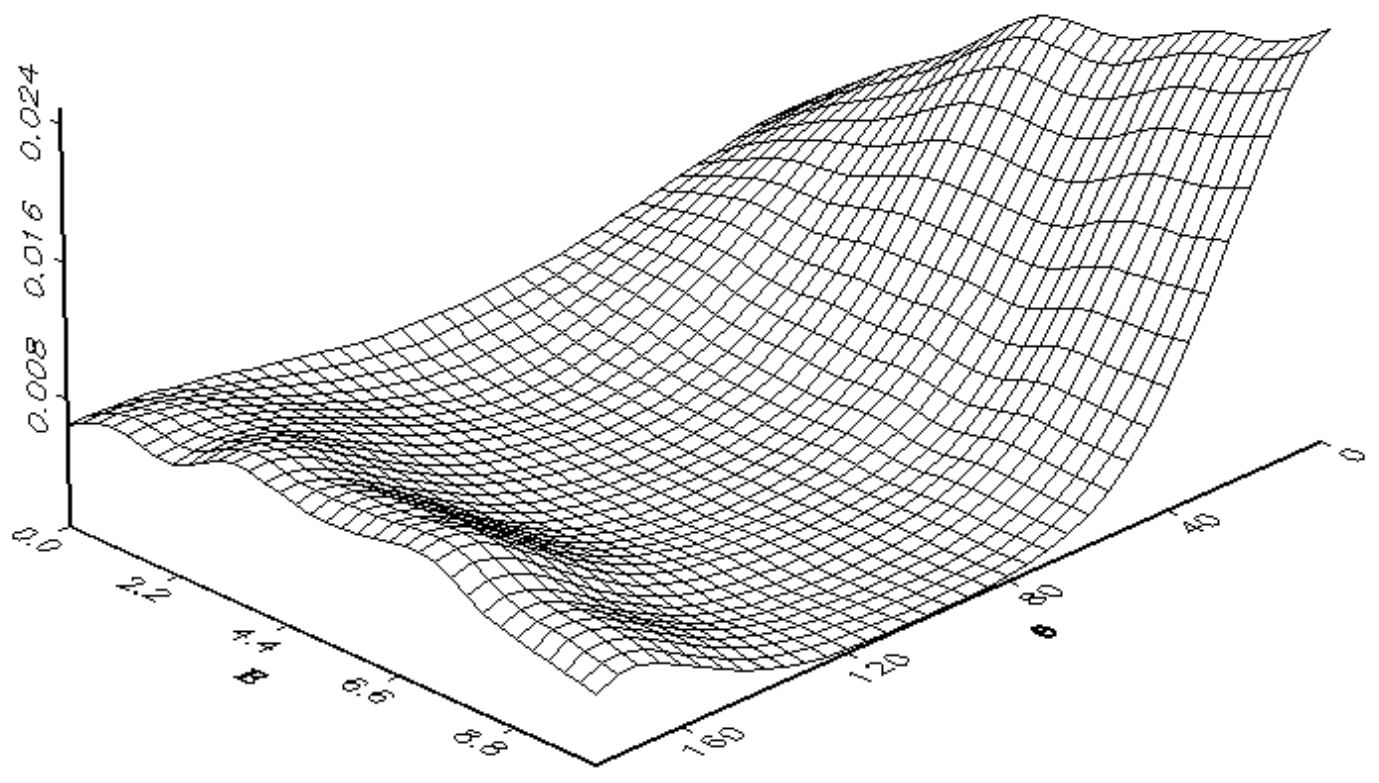

Figure 2.4. Probability density function for angular distribution (lab system) of neutrons from fission with respect to the direction of the light fragment 


\subsubsection{Fission Neutron Energy Distribution}

The neutron energy spectrum is obtained from the nuclear cross-section files for induced fission. A corrected Maxwellian distribution is used for the prompt neutron energy spectrum for ${ }^{252} \mathrm{Cf}$. A detailed analysis of the experimental data has been performed by Mannhart ${ }^{21}$ to obtain the relative difference between the neutron spectrum obtained from the measured data and the Maxwellian distribution for the spontaneous fission of ${ }^{252} \mathrm{Cf}$. Mannhart developed an energy dependent correction factor for the Maxwellian distribution. The fit to the correction factor data can be found in Appendix C. Multiplying the Maxwellian distribution by the correction factor, an accurate representation of the energy distribution of neutrons from the spontaneous fission of ${ }^{252} \mathrm{Cf}$ is obtained. The average energy obtained from the corrected distribution is $2.13 \mathrm{MeV}$, which corresponds to the measured value of the average energy of neutrons from the spontaneous fission of ${ }^{252} \mathrm{Cf}$. There is an option in the code to use the theoretical spectra of Madland and $\mathrm{Nix}^{22}$ or the Watt fission spectra from Froehner ${ }^{23}$ for ${ }^{252} \mathrm{Cf}$ fission. An eight-point Gaussian quadrature integration scheme was used to integrate the spectrum from zero to an upper limit of $E$, where $E$ was varied from $0.00001 \mathrm{eV}$ to $25 \mathrm{MeV}$. The integrated spectrum was then normalized to unity. The neutron energy is selected by setting a random number equal to the normalized integrated spectrum and then determining the energy that corresponds to this value of the normalized integrated spectrum. A least-squares polynomial fit of the neutron energy as a function of the value of the normalized integrated spectrum was used. This resulted in an expression for the neutron energy as a function of the normalized integrated spectrum, that is, the neutron energy as a function of the random number. A Watt fission spectrum is used for the spontaneous fission of the inherent fission sources. The parameters for the Watt spectrum can be found in Appendix C.

\subsection{MODIFIED GAMMA RAY TRANSPORT}

In MCNP, gamma ray production from neutron events is determined by sampling a total gamma ray production cross section. These cross sections contain data on the multiplicity and energy of gamma rays from neutron reactions that produce gamma rays. These reactions include neutron capture, inelastic neutron scattering, and fission. Although the cross sections are measured separately, these gamma-ray-producing reactions are lumped together in the MCNP nuclear data sets. Consequently, these data cannot be easily separated without extensive modifications to the code and the formatted data files. This grouping does not allow the physics of the gamma ray production process to be exact on an event-by-event basis but does give a correct average behavior. ${ }^{24}$ This will lead to no uncertainty in the first moment of the populations but may introduce some uncertainty into the second moment on which noise-measured quantities depend, especially when inelastic neutron scattering and fission are equally probable. This may also reduce some of the fluctuating phenomena associated with gamma ray production. The multiplicity and energy distribution of prompt gamma rays from the spontaneous fission of the ${ }^{252} \mathrm{Cf}$ source has been included in MCNP-DSP. 


\subsubsection{Prompt Gamma Ray Multiplicity for ${ }^{252} \mathrm{Cf}$}

The gamma ray multiplicity, like the neutron multiplicity, is a function of the fission fragment mass. A saw-tooth dependence of the gamma ray yield on fragment mass has been observed for the spontaneous fission of ${ }^{252} \mathrm{Cf}$ and for thermal neutron fission of ${ }^{235} \mathrm{U} \cdot{ }^{26}$ Because the number of gamma rays emitted is mainly dependent on the fission fragment properties, a single distribution can be used to describe the gamma ray multiplicity for ${ }^{252} \mathrm{Cf}$.

The gamma ray multiplicity was determined using Brunson' ${ }^{26}$ measurements, which fitted the data to a double Poisson model. Brunson's model depends on the minimum energy of the gamma rays emitted. For a minimum energy of $85 \mathrm{keV}$, the resulting probability distribution is used to obtain the gamma ray multiplicity

$$
\Pi(G)=0.682 \frac{7.20^{G} e^{-7.20}}{G !}+0.318 \frac{10.71^{G} e^{-10.72}}{G !} .
$$

where $G$ is the gamma ray multiplicity. The gamma ray multiplicity ranges from 0 to 20 gamma rays per fission with an average value of 8.32 gamma rays per fission. This value does not differ greatly from that obtained by others. ${ }^{25}$

\subsubsection{Prompt Gamma Ray Energy Distribution for ${ }^{252} \mathrm{Cf}$}

The gamma ray energy spectrum has been measured for the spontaneous fission of ${ }^{252} \mathrm{Cf}$ and the thermal-neutron-induced fission of ${ }^{235} \mathrm{U}$. There appears to be little difference between the spectrum from ${ }^{252} \mathrm{Cf}$ and that from ${ }^{235} \mathrm{U} \cdot{ }^{27}$ Because of the small difference between the two spectra and because the measurements of the ${ }^{235} \mathrm{U}$ gamma ray spectra are more precise, the gamma ray spectra from the thermal neutron fission of ${ }^{235} \mathrm{U}$ is used to obtain the gamma ray energy.

The energy spectrum of the prompt fission gamma rays is obtained from Maienschein's measurements. ${ }^{28,29}$ The distribution, from 0.3 to $1 \mathrm{MeV}$, is described as

$$
N(E)=26.8 e^{-2.30 E} .
$$

In the interval 1.0 to $8.0 \mathrm{MeV}$, the distribution is described as

$$
N(E)=8.0 e^{-1.10 E} .
$$

The upper energy limit of $8 \mathrm{MeV}$ was selected because nuclear excitation above $8 \mathrm{MeV}$ typically leads to neutron rather than gamma emission. Although Maienschein's experiment determined the gamma ray spectrum down to $0.25 \mathrm{MeV}$, there are several gamma ray energies that show preferential emission for low multiplicities below 0.3 $\mathrm{MeV}^{26}$ On the advice of Ray Nix ${ }^{20}$ the spectrum below $0.3 \mathrm{MeV}$ is represented as 


$$
N(E)=38.13(E-0.085) e^{1.648 E} .
$$

The minimum gamma ray energy of $0.085 \mathrm{MeV}$ coincides with Brunson's measurements. Below this energy, gamma ray emission is due to K-shell X-rays from the fission fragments that occur later than the prompt gamma emission from fission. Using this functional representation of the prompt fission gamma ray energy spectrum, a value of $0.898 \mathrm{MeV}$ is obtained for the average gamma ray energy per fission. This value agrees well with the accepted value of $0.88 \mathrm{MeV}$ per fission. ${ }^{26}$

\subsubsection{Prompt Gamma Ray Multiplicity for Spontaneous Fission Isotopes}

Measured data for the multiplicity distributions of prompt gamma rays from various spontaneous fission isotopes do not exists. However, an approximation was used to determine such distributions. ${ }^{30}$ Nifenecker ${ }^{31}$ has observed that there is competition in the de-excitation mechanism of the fission fragments. This competition in the de-excitation process results in a correlation between the total gamma ray energy release and the number of neutrons emitted from fission. The correlation between the neutron and gamma ray emission has been used to determine the average number of prompt gamma rays from fission. A negative binomial distribution has been used to describe the multiplicity distribution functions. The approximated gamma ray distribution parameters are given in Appendix D. These distributions should only be used for sensitivity studies and are not to be used for benchmark calculations because the multiplicity distributions are only estimates and are not measured data. Furthermore, the energy spectra of prompt fission gamma rays are simply equated to that for ${ }^{252} \mathrm{Cf}$ because such spectra have not been measured.

\subsection{RADIATION DETECTION}

Although MCNP is capable of tracking electrons, the electron production and subsequent light production in the detectors were not treated rigorously. An intuitive approach was used to determine when particles contribute to the detector response. This approximation faithfully reproduces the measured detector responses. Currently, three detector types can be employed in a calculation: capture, scatter, or fission detectors.

\subsubsection{Neutron Detection}

In the MCNP-DSP calculation, neutron detection is characterized by a particular neutron event. The detector response of capture detectors is primarily due to the absorption of a neutron with the subsequent emission of secondary charged particles, which ionize the detection media and produce an electronic pulse whose amplitude is proportional to the kinetic energy deposition of the secondary charged particle. These detectors typically have a very large thermal neutron absorption cross section and a very small scattering cross section. In MCNP-DSP, if a neutron is absorbed in a capture detector, a count is scored in the appropriate time bin, and the number of detection events is incremented. 
Scattering detectors are those in which the response is due primarily to neutron scattering in the detection media. To observe a count in a scattering detector, the neutron must transfer a certain pre-selected amount of energy to the detection material via the kinetic energy of the recoil nucleus. In the actual detector this recoil nucleus excites electrons in the scintillation material to produce light that is converted into an electrical pulse by a photomultiplier tube. Although MCNP can handle this scintillation process directly, an intuitive approximation was utilized which assumes that the light production is proportional to the energy deposited by the neutron. An energy threshold is specified in the calculation for each detector. Since multiple neutron scattering can occur in the detector, special attention was given to modeling the multiple scattering in these detectors by specifying a time width, the pulse generation time, in which energy contributions from the events are summed together. If a neutron scatters in the detector and deposits an amount of energy greater than the neutron threshold, a count is registered, and additional neutron scatters within the specified pulse generation time of the detector are ignored. However, if the amount of energy deposited in the detector is less than the neutron threshold, the energy of the subsequent neutron scatters in the detector are added together for those events that occur within the pulse generation time of the detector. To account for neutrons that may scatter between adjacent detectors several times, the time at which the neutron had its last scattering event in each detector is stored for each neutron track.

In fission detectors, the fission fragments travel through the detection media, ionizing the atoms in the detector. The large energy release per fission allows for easy discrimination of other events that may also produce ionized atoms in the detector. In the calculation, a count is registered each time a fission event occurs in the detection media, and the fission neutrons are stored for tracking.

\subsubsection{Gamma Ray Detection}

Various gamma ray events may lead to a detection event in the capture and scattering detectors since the gamma ray events also produce secondary charged particles. If the gamma ray energy deposited is above a certain threshold, which is specified as input to the calculation, gamma ray absorption due to the photoelectric effect can lead to a detection event. Likewise, if the gamma ray has an incoherent scatter in the detector and the recoil electron energy is greater than the threshold, a detection event will occur. Multiple scattering of gamma rays in the detector media is treated much in the same way as for neutrons.

\subsubsection{Dead Time Models}

An option has been included in the code to simulate the dead time in a detector. The dead time is specified for each detector. If the detector is a composite detector then the largest dead time is selected for this detector. Two options are available for the dead time models. The first option is a non-paralyzed model in which the dead time for a given count only extends a period of time equal to the dead time width. The second option is a paralyzed model in which the dead time for a given count can be extended if subsequent counts fall within the dead time of the initial detector pulse. 
The dead time correction is performed after each block of data is sampled. The dead time is divided by the time-bin width to determine the number of time-bins over which the dead time extends. The time-bin arrays for each detector are modified by removing counts in time-bin array that occur during the dead time period using either the paralyzed or non-paralyzed model. The dead time correction will not be made if the dead time period is less than the width of the time bins. 


\section{CALCUlational MODES}

There are six calculation modes that are available with MCNP-DSP. These are source-driven noise analysis, passive noise analysis, correlation analysis (Rossi- $\alpha$ ), pulsed neutron calculations, Feynman variance, and multiplicity simulation. All of these modes of analyses are related. This chapter provides a brief description of each of these simulation modes.

\subsection{TIME DECIMATION OF SIGNALS}

In subcritical measurements, the detector counts are accumulated and stored in time intervals. For example, the detector response may be sampled every nanosecond for 1000 nanoseconds. One sample of the detector response is treated as a data block. A data block is acquired synchronously in time for each detector and/or source. The discrete data blocks are processed in various ways depending on the measurement method. The detector responses for each data block are processed to obtain estimates of the frequency spectra, the correlation functions, the multiplicity data, the variance-to-mean data, or the pulsed neutron response.

In the simulation, the number of time intervals is specified along with the sampling rate. The width of each time interval is the inverse of the sampling rate; therefore, the total time period is simply the product of the number of time bins with the inverse of the sampling rate. The interactions in the detectors are tracked to determine the time bin in which the detector count occurred. Likewise, the event times for the source also determine the bin in which the source event occurred. The source may either be an active source or a passive source. Multiple source types are selectable for either active or passive simulations. For active simulations, the time of the source event may occur at time zero or may occur randomly in the time block. For passive simulations, the source events occur randomly within the time block. The total time period is referred to as a data block. The detector responses for each data block are processed to obtain estimates of the frequency spectra, the correlation functions, the multiplicity data, the variance-to-mean data, or the pulsed neutron response as is done in the measurements.

\subsection{FREQUENCY ANALYSIS SIMULATION}

Frequency (noise) analysis measurements can be performed either actively or passively. Active measurements require the use of a timed neutron source whereas passive measurements rely upon an inherent source in the fissile configuration to initiate the fission process in the material. Data acquisition in noise analysis measurements requires a variety of electronic components. The electronic system consists of components that are used to convert the detector signals to an analog signal that can be digitized by the Fourier analyzer. The detector pulses are typically input into an amplifier to increase the voltage of the pulses from the detectors. The signal from the amplifier is then sent to some type 
of discriminator to eliminate any unwanted pulses. Pulses from the discriminator are then directed to a resistor-capacitor (RC) filter to shape the pulse from the discriminator. The $\mathrm{RC}$ filter is an integrator that integrates the pulse using a selectable time constant and adjustable gain. The RC filter is used for pulse mode operation of detectors. The signal is then sent to the Fourier analyzer where the signal is digitized. The Fourier analyzer uses low-pass filters to eliminate the upper frequency components that are greater than half the sampling rate. This prevents aliasing from occurring during the discrete Fourier processing of the signals. Aliasing is the folding of high frequencies over to the low frequency when the sampling rate is less than twice the maximum frequency of the signal. ${ }^{32}$ The filtered signal is digitized repeatedly by an analog-to-digital converter to provide an integer value proportional to the voltage of the signal. The digitized data are divided into segments, termed data blocks, of typically 512 or 1024 points, and then the data are Fourier transformed. Auto and cross spectra are calculated for each data block by complex multiplication of the Fourier transformed data.

The auto spectrum is defined as

$$
G_{x x}(\omega)=X^{*}(\omega) X(\omega)
$$

where $X(\omega)$ is the Fourier transform of the detector signal and * denotes the complex conjugate. Similarly, the cross spectrum between two signals $x(t)$ and $y(t)$ is defined as

$$
G_{x y}(\omega)=X^{*}(\omega) Y(\omega)
$$

Estimates for the auto and cross spectra are computed for each data block and are then averaged with the previous data to obtain the current auto and cross spectra. This process is continued until the desired convergence is reached.

The frequency analysis simulation incorporates some of the filtering and windowing options used in measurements. To simulate the band-pass filter, two filter options can be employed in the calculation. Rockland and Precision filters were used in the measurements; hence, functions that represent these filters may be used in the calculations. The Rockland filter function is shown in Fig. 3.1, and the Precision filter function is presented in Fig. 3.2. The peak values of the filter functions are normalized to one. The upper frequency cutoff is set equal to $40 \%$ of the sampling rate as implemented in the measurement to prevent aliasing. These filter functions are applicable only for sampling rates less than $100 \mathrm{kHz}$ because the low-pass filter has not been included in the model and its time response affects the detector response for sampling rates greater than $100 \mathrm{kHz}$. Other higher frequency filter functions could easily be incorporated into the code if the waveforms are available. The time when a particle is detected is determined by adding its time of birth to the time that the particle spent in the system before being detected. A count is registered in the appropriate time bin whose width is determined from the inverse of the sampling rate. If the filter functions are used, the pulse is spread out over approximately ten time bins for each detection event. For each time bin, the filter function is segmented into 
ten discrete values that begin at the time of detection. The digitized values of the normalized filter function that occurs at the right boundary of the time bin are added to the detector time response for that bin. If the particle detection occurs near the end of the data block, the digitized values in the time bins beyond the length of the data block are ignored. The low-frequency components of the detector signal are eliminated by ac coupling (highpass filtering) in the experiments. To simulate the implementation of a high-pass filter, the average counts per bin may be subtracted for each detector's time spectrum for each data block. The removal of the mean value is performed independently of the implementation of the low-pass filter. Three commonly used windows may be employed in the calculation.

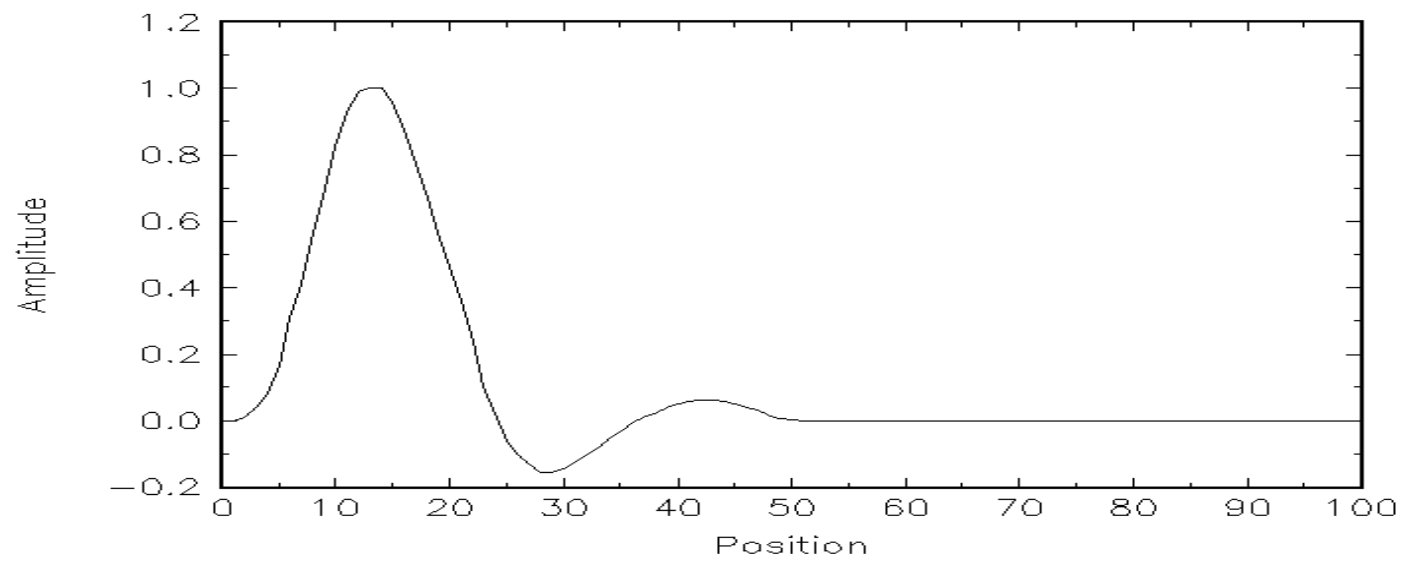

Figure 3.1. Rockland filter function

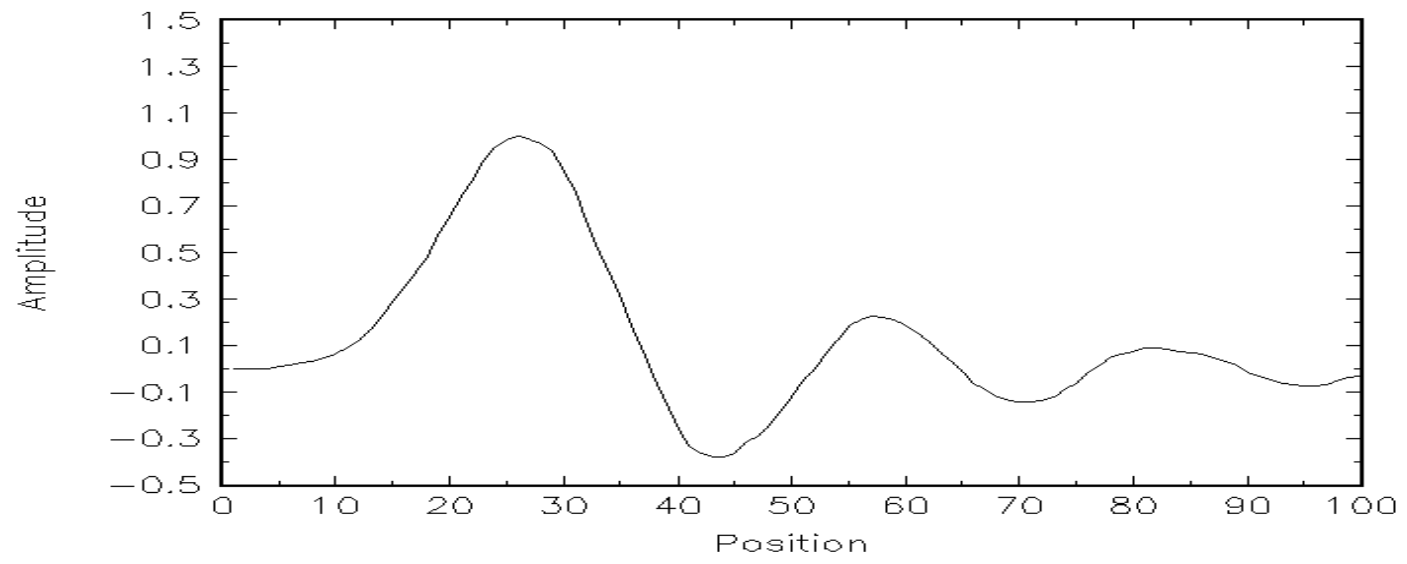

Figure 3.2. Precision filter function

Windows are applied to the time spectra to correct for errors due to the finite length of the data block that coincides with a finite length Fourier transform. The windowed time signal is given by 


$$
x^{\prime}(n)=x(n) w(n),
$$

where $x(n)$ is the discrete detector time signal, and $w(n)$ is the window function. Bartlett, Hanning, and Hamming windows are available in the calculations and are given by ${ }^{32}$

$$
\begin{array}{cl}
\begin{array}{l}
2 n / N, \\
2-2 n / N,
\end{array} & \begin{array}{l}
0 \leq n \leq N / 2 \\
0,
\end{array} \\
w(n)=n \leq N \\
\begin{array}{ll}
0.5-0.5 \cos (2 \pi n / N), & 0 \leq n \leq N \\
0, & \text { otherwise }
\end{array} \\
w(n)=\begin{array}{ll}
0.54-0.46 \cos (2 \pi n / N), & 0 \leq n \leq N \\
0, & \text { otherwise }
\end{array}
\end{array}
$$

where $N$ is the length of the data block. A Hanning window has been used in some noise analysis the measurements. A discrete Fourier transform is applied to the data block of length $N$. The discrete Fourier transform (DFT) is defined as

$$
X(k)={ }_{n=0}^{N-1} x(n) W_{N}^{k n},
$$

where $W_{N}^{k n}=e^{-j 2 \pi k n / N}$ is the complex quantity. Because the DFT is a complex function, the estimates of the auto and cross spectra are obtained by complex multiplying the DFT of the detector responses for each data block. The auto and cross spectra are calculated for each data block and averaged with the values from the previous data blocks.

\subsubsection{Source-Driven Noise Analysis Simulations}

Source-driven noise analysis simulations require selection of a source and two or more radiation detectors. The simulation follows the natural chain of events. The source starting time is randomly selected over the time period of the data block. The source particles and their progeny are tracked until they are absorbed or leak. Interactions with the detectors can produce counts in the time bins. The time when a particle is detected is determined by adding its time of birth to the time that the particle spent in the system before being detected. A count is registered in the appropriate time bin whose width is determined from the inverse of the sampling rate. The time responses of the source and detectors are accumulated for each data block. After all particles for a given block have been tracked, the source and detector responses are processed. If the filter and window functions are invoked, the time dependent source and detector responses are adjusted. The time responses are then Fourier transformed. The auto and cross spectra are defined by 
performing a complex multiplication of the Fourier transform of the data blocks of detector signals. The auto and cross spectra are estimated for each data block, and the average value is accumulated over many data blocks.

\subsubsection{Passive Noise Analysis Simulations}

Passive noise analysis simulations rely upon an internal source such as an inherent spontaneous fission source to produce fission events within the system. Therefore, a source signal is not acquired in the passive simulations. In passive measurements, the inherent source produces neutrons and/or gamma rays at random times within the data block. Therefore, in the passive noise analysis simulation, the source events occur randomly within the detector time-response data block. The source events occur in any time bin with equal probability. The passive noise analysis computation can be used to simulate break frequency noise analysis measurements. The source particles and their progeny are tracked until they are absorbed or leak. Interactions with the detectors can produce counts in the time bins. The time when a particle is detected is determined by adding its time of birth to the time that the particle spent in the system before being detected. A count is registered in the appropriate time bin whose width is determined from the inverse of the sampling rate. The time responses of the detectors are accumulated for each data block. After all particles for a given block have been tracked, the detector responses are processed. If the filter and window functions are invoked, the time dependent detector responses are adjusted. The time responses are then Fourier transformed. The auto and cross spectra are defined by performing a complex multiplication of the Fourier transform of the data blocks of detector signals. The auto and cross spectra are estimated for each data block, and the average value is accumulated over many data blocks

\subsection{CORRELATION ANALYSIS SIMULATION}

Correlation (Rossi- $\alpha$ ) measurements require the use of a time analyzer for recording detector pulses. The pulses from the detectors are stored into time bins. The time distribution of counts in one channel with respect to another channel is accumulated. This process is repeated for many samples of the detector responses to obtain a histogram. The auto correlation (single-detector Rossi- $\alpha$ ) measurement is performed if the same detector signal is placed in both channels. Likewise, the cross correlation (two-detector Rossi- $\alpha$ ) measurement if performed if different detector signals are placed in the analyzer channels.

The correlation simulation is a calculation of the Rossi- $\alpha$ measurement. As with the frequency analysis simulation, the correlation simulation can be performed actively or passively. The source particles and their progeny are tracked until they are absorbed or leak. Interactions with the detectors can produce counts in the time bins. The time when a particle is detected is determined by adding its time of birth to the time that the particle spent in the system before being detected. A count is registered in the appropriate time bin whose width is determined from the inverse of the sampling rate. The time responses of the detectors are accumulated for each data block. After all particles for a given block have been tracked, the detector responses are processed. The correlation functions are actually 
obtained from the frequency analysis signatures. The inverse Fourier transforms of the auto spectra are used to obtain the auto correlation functions that are the equivalent of the single detector Rossi- $\alpha$ measurements. Likewise, the inverse Fourier transforms of the cross spectra are used to obtain the cross correlation functions. The cross correlation function between two detectors is equivalent to the two detector Rossi- $\alpha$ simulation. The process for generating the correlation functions is as follows. The detector time responses are acquired for one block of data. The data block is then zero padded (i.e. the length of the data block is doubled by adding zeros to the block). The zero padding is necessary to ensure that the correlation functions obtained during the inversion process are linear. The zero padded time responses are Fourier transformed. The Fourier transformed data are then complex multiplied to obtain the auto and cross spectra. The auto and cross spectra are averaged for many data blocks. When the computation is completed, the auto and cross correlation functions are obtained by inverse Fourier transformation of the auto and cross spectra. Therefore, the correlation functions are not generated until the computation acquires the desired number of data blocks or the computation time limit is reached.

\subsection{PULSED SOURCE SIMULATION}

A pulsed source measurement requires the use of a neutron and/or gamma ray source and an appropriate detector. The signals from the source indicate emission of neutrons and/or gamma rays. The counts in the detector occur some time after the initiating source event. The pulsed source measurement can be performed with a variety of electronic components. One way to perform a pulsed source measurement is to use a timeto-amplitude converter (TAC) that is triggered with the source event and stopped with a detector signal. The TAC output is sent into a multi-channel analyzer to obtain the neutron and/or gamma ray time-of-flight.

The pulsed source measurement is simulated by starting the source particles at time zero and tracking the source particles and their progeny to the detector. The source particles and their progeny are tracked until they are absorbed or leak. Interactions with the detectors can produce counts in the time bins. The time when a particle is detected is determined by adding its time of birth to the time that the particle spent in the system before being detected. A count is registered in the appropriate time bin whose width is determined from the inverse of the sampling rate. The time of detection for each particle is determined and the counter for the appropriated time bin is incremented much like the accumulation of the pulse height spectra with a multi-channel analyzer. For a single "hit" measurement system, only one detector count triggers the TAC for each source event; however, in the calculations, all detector counts for a given source event are stored. The dead time options cannot be used in the pulsed source simulation because of the process by which the detector counts are accumulated. Users who wish to perform a pulsed source simulation using the dead time options should perform a correlation calculation in the active mode to obtain the correlation between the source and the detector and force the starting times of the particles to begin at time zero. 


\subsection{FEYNMAN VARIANCE SIMULATION}

Feynman variance measurements require a time analyzer to measure the number of detector counts in a data block from which the variance of the counts is estimated. The process is repeated for data blocks of different length $(T)$. A plot of the reduced variance versus the length of the data blocks is used to determine the prompt neutron decay constant.

The Feynman variance simulation requires the computation of the average number of counts and the average of the counts squared for a given time period. As in the other simulation modes, the simulation begins with the tracking of the source particles and concludes when all source particles and progeny have been tracked for a given data block. The detector events are determined for each data block. In the simulation the average counts per block and average counts squared per block for the detectors are computed for each block and averaged with the previous block. The reduced variance can be computed from the average counts per block and the average squared counts per block. The calculations must be repeated with varying sampling rates to determine the variation of the reduced variance versus the length of the data block. The dead time options can be invoked with the Feynman variance simulation.

\subsection{MULTIPLICITY SIMULATION}

The multiplicity measurement requires the use of a time analyzer to determine the number of counts that occur after in a detection channel after a trigger event. The trigger event could be a count from another detector, a count from the source, or a count in the same detector. The measurement is performed to determine the number of times zero, one, two, etc., counts occur after a trigger event. The distribution of these counts is directly related to the reactivity of the system. In the measurements, the random number of counts per time period is also determined.

The multiplicity simulation determines both the detector triggered and random number of counts for each data block. The distribution for random counts is determined as the number of counts that occur in the data block. The distribution for triggered counts is obtained by determining the number of counts after the trigger count for the same time interval. Hence, the time period for triggered counts is potentially twice as long as that for the random counts. Therefore, particles are tracked for twice the period of the data block.

This process is best described using the illustration in Fig. 3.3. The illustration in Fig. 3.3 represents one sample of the detector response where the arrow is the time period of one data block. In this case, four counts occurred in the time interval. Therefore, the distribution histogram for four random counts would be incremented by one. Four trigger counts are obtained for this sample of the detector response. The counts in the next data block after the current must also be determined to create the triggered distribution. The triggered distribution for five counts would be incremented by three: one for the first 
trigger interval, one for the second trigger interval, and one for the third trigger interval because five counts occur within the sample time interval after the trigger event. Finally, the trigger distribution for four counts would be incremented by one for the fourth trigger interval. This process is repeated over many samples of the detector response to obtain triggered and random distribution histograms. Note that these "multiplicity" distributions do not count the trigger pulse as one of the events.

\section{Triggered}
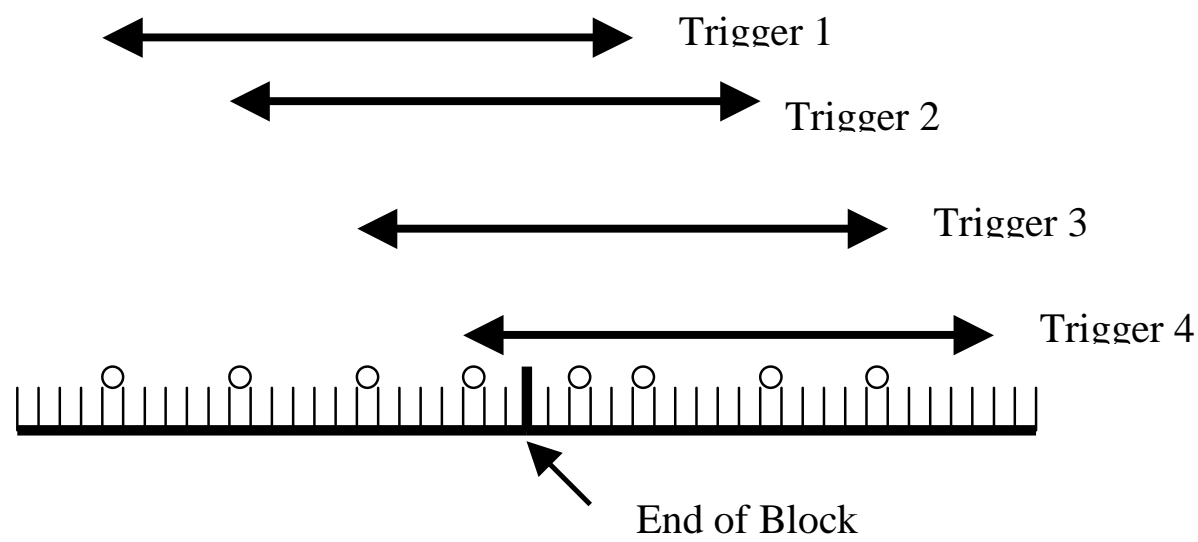

Random

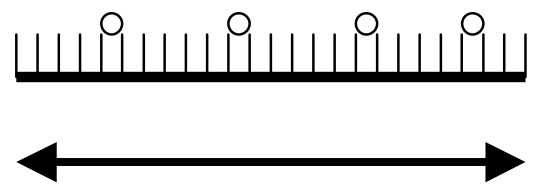

Figure 3.3. Diagram of multiplicity simulation. 


\section{MCNP-DSP PROGRAM FLOW}

The general structure of MCNP has been preserved as much as possible. However, some modifications had to be made in order to obtain the detector responses in a manner similar to the measurement. The general flow of MCNP4A is shown in Fig. 4.1. The program starts by reading the command line to determine which options the code uses. After determining the options, the program begins execution. The first step is to read the input file, which can be stated on the command line or the default input file name, INP. The arrays are initialized with a call to subroutine $I M C N$. If a subcritical simulation is to be performed, a separate data file containing options for the subcritical simulation is read in $I M C N$. After initializing the arrays, the interactive geometry plot subroutine PLOTG is called if the command line contains $I P$ and the options to plot the geometry. Next, the cross-section data are read into the appropriate arrays. Once the cross-section data have been placed in the arrays, the code begins the actual tracking of the particles by calling subroutine MCRUN. Subroutine MCRUN runs the particle histories by calling TRNSPT, which, in turn, calls HSTORY to follow the particle tracks throughout the system and writes the detector information. Finally, the tallies are plotted with a call to MCPLOT after all the particles have been analyzed. This general structure has been maintained in the modified code. The major difference occurs in the actual transporting of the particles. The subroutine TRNSPT has been modified to call HSTORYCF if a subcritical calculation is to be performed.

The structure of the subcritical simulations is based on an outer and an inner loop. The outer loop controls the number of blocks of data ( $b k s)$ to be calculated. The average number of source events per data block ( $s c d$ ) controls the inner loop. If the inherent spontaneous fission source option is invoked, there is a second inner loop that is controlled by the average number of inherent spontaneous fissions per block $(s f p d)$. Because spontaneous fission is a Poisson process, the number of disintegrations per block (ncdis) is calculated from a Poisson distribution with mean $s c d$ for the source events per block for a spontaneous fission source and with mean $s f p d$ for the inherent fission source fissions per block. For noise analysis simulations, the ratio of the source rate to the inherent source fission rate must be known to specify $s c d$ and $s f p d$. For source-driven noise analysis simulations, the average number of source events per data block is related to the actual source size by the following relation ${ }^{5}$

$$
s c d=\frac{m R_{s f} n t b n}{f_{s}},
$$

where $m$ is the mass of the source in micrograms, $R_{s f}$ is the spontaneous fission rate of the source per microgram, $n t b n$ is the number of points per block, and $f_{s}$ is the sampling rate. Using this expression, the source specified in the calculation can be related to the actual source used in the measurements. 


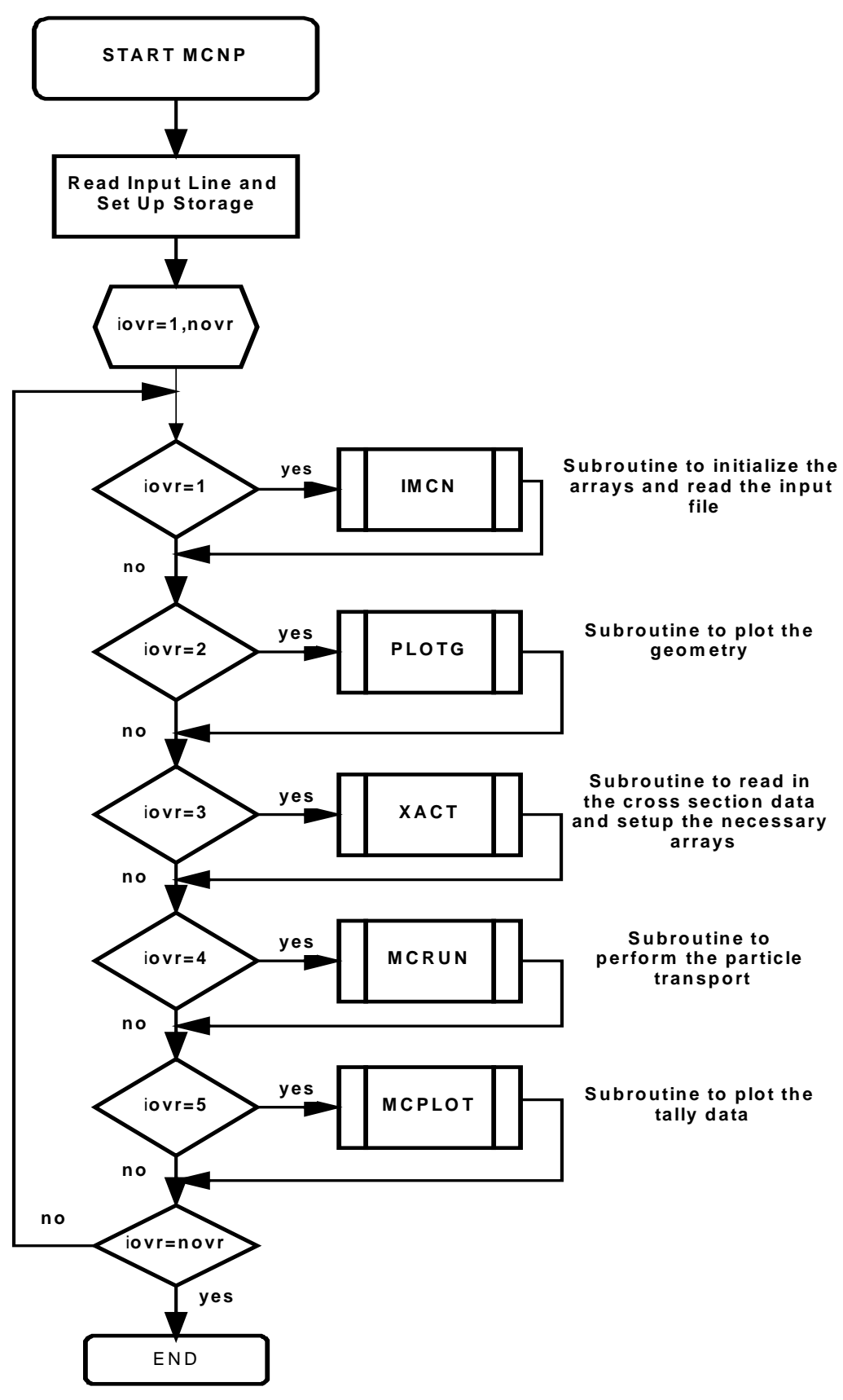

Figure 4.1. General MCNP4a program flow

The particle tracking begins with the first data block. First, the source particles and their progeny are tracked. Next, the particles from the inherent fission source and their progeny are tracked if this option is invoked. When the inner loops are finished, the block data are processed. This processing could be the direct calculation of the frequency spectra or the calculation of the correlation functions. After the block data have been processed, the outer loop variable is incremented and the inner loops are restarted. When all of the 
data blocks have been calculated, the outer loop is completed. The summary information is written to the output files. This process is depicted in Fig. 4.2.

Because the inner loops control the actual particle transport, a detailed description of the particle tracking follows. The inner loop begins by obtaining information about the source particles. The position is specified in the extra data file for the source particles and is chosen randomly in a specified volume for the inherent fission source. The time of the spontaneous fission is chosen randomly in the data block or starts at zero depending on user specifications. The number of particles from the source is determined. For source-driven noise simulations using ${ }^{252} \mathrm{Cf}$, Spencer's distribution is used to obtain the number of prompt neutrons, and Brunson's distribution is used to determine the number of prompt gamma rays. Zucker and Holden's data are used to determine the number of prompt neutrons from the inherent spontaneous fission source. The energies of the ${ }^{252} \mathrm{Cf}$ neutrons are chosen from the corrected Maxwellian distribution, and the energies of the ${ }^{252} \mathrm{Cf}$ gamma rays are chosen from Maienschein's spectrum. The energy spectra of the inherent fission source neutrons are chosen from the usual MCNP Watt fission spectrum. The direction of the source neutrons can be chosen isotropically or from the angular distribution data of BudtzJorgensen and Knitter. All particles but one are stored in the bank. An overview of the particle tracking is shown schematically in Fig. 4.3. The distance to the cell boundary, $d_{b}$, is determined from the particle's starting location and the direction cosines. Next, the distance to the collision site, $d_{c}$, is calculated from the usual relation $d_{c}=-\ln R / \Sigma_{t}$, where $R$ is a random number and $\Sigma_{t}$ is the total macroscopic cross section. The minimum distance determines whether or not the particle has a collision. If $d_{b}$ is less than $d_{c}$, the particle is transported through the cell boundary, where the distance to the next cell boundary and the distance to collision are calculated for the new cell. If the particle enters a region of zero importance (outside the system), the particle is said to have leaked, and the leakage tallies are updated. If $d_{c}$ is less than $d_{b}$, then the particle collides in the cell. When a particle collides, the collision nuclide is selected randomly from the materials present in the cell. If the particle is a neutron, the velocity of the collision nuclide is calculated, and the collision event is determined. The capture probability is calculated in the usual way. If the problem is a dual neutron-gamma ray calculation, gamma rays may be produced by the neutron interaction. If the particle is not lost by capture, the neutron will have either an elastic collision or an inelastic collision. ${ }^{(a)}$ The probability of having an elastic collision is given by $\sigma_{e l} l\left(\sigma_{e l}+\sigma_{i n}\right)$, where $\sigma_{e l}$ is the microscopic elastic scattering cross section and $\sigma_{i n}$ is the microscopic inelastic cross section. The type of inelastic event, $n$, is determined from

$$
{ }_{i=1}^{n-1} \sigma_{i}<\xi_{i=1}^{N} \sigma_{i} \leq{ }_{i=1}^{n} \sigma_{i}
$$

where $\xi$ is a random number on the interval [0 to 1$), N$ is the number of inelastic reactions, and the $\sigma_{i}$ 's are the inelastic reaction cross sections at the incident neutron energy. ${ }^{3}$

\footnotetext{
(a) The term inelastic collision is a carryover from MCNP and means all reactions except elastic scattering and capture.
} 


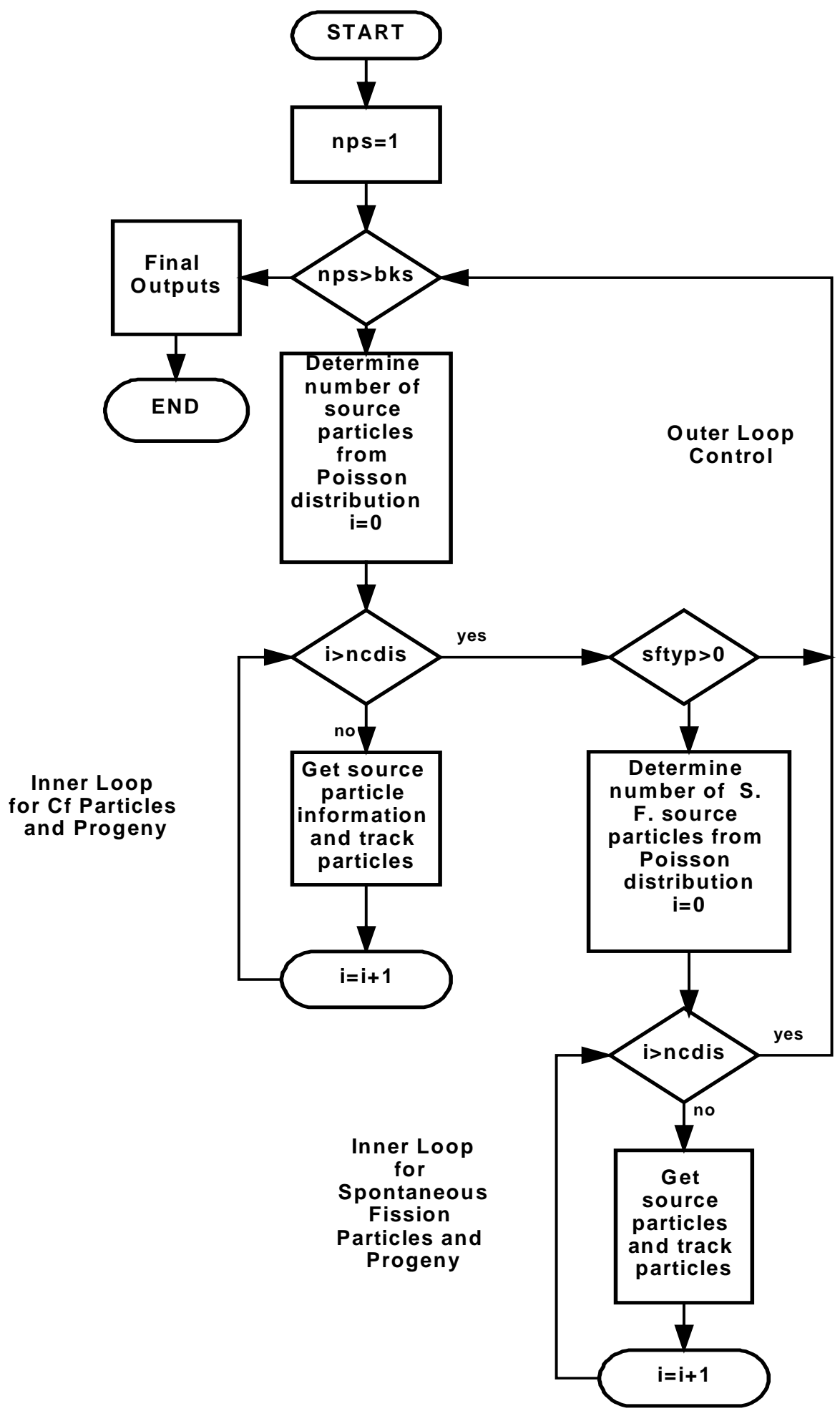

Figure 4.2. Structure of inner and outer loops of MCNP-DSP 


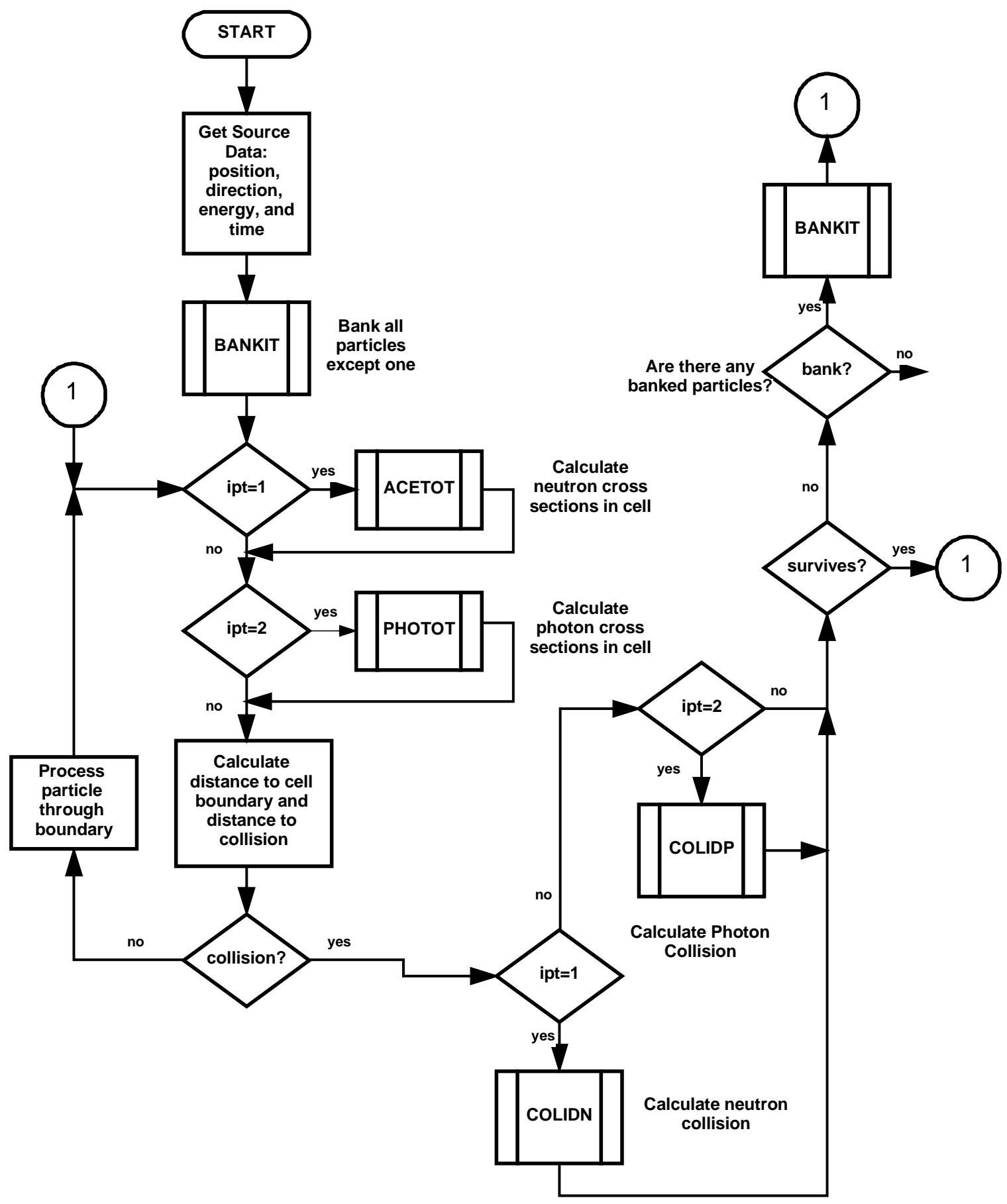

Figure 4.3. Particle tracking procedure

If the inelastic event is fission, the number of neutrons produced from fission is determined from Gwin et. al. data, Zucker and Holden's data, or from Terrell's formula, depending on the fission isotope. The energy of the emerging neutrons is sampled from the appropriate spectrum indicated in the cross-section data file. The direction cosines may be either isotropic or determined from the experimental angular distribution data. Data for neutrons 
produced from fission are stored in the bank to be tracked later. If the particle is a gamma ray, the determination of the type of interaction is the same as that used for neutron collisions. However, the collision physics are different. ${ }^{3}$ In the modified code, the detailed physics treatment is used by default. The detailed physics treatment includes incoherent scattering, coherent scattering, the photoelectric effect, and pair production. Therefore, the total gamma ray cross section, $\sigma_{t}$, is

$$
\sigma_{t}=\sigma_{i c}+\sigma_{c o}+\sigma_{p e}+\sigma_{p p},
$$

where $\sigma_{i c}$ is the incoherent scattering cross section, $\sigma_{c o}$ is the coherent scattering cross section, $\sigma_{p e}$ is the photoelectric cross section, and $\sigma_{p p}$ is the pair production cross section. The selection of the gamma ray event is determined in a manner similar to that of the neutron events.

The tracking and collision process is repeated for each particle until the particle either leaves the system or is absorbed by analog capture. Next, particles are retrieved from the bank, and the tracking process proceeds as before until all the source particles and their progeny have been tracked for the given data block. Then, the particles for the inherent fission source are tracked if the spontaneous fission source option is invoked. The inner loops are now complete for one data block, and the detector responses are then processed. After processing the detector responses, the outer loop variable is incremented, and the inner loop procedure is repeated. After completing the desired number of blocks, the program calculates the final estimates of the desired quantities and writes the data to a file. 


\section{INPUT OPTIONS}

To perform the MCNP-DSP calculations, the idum and files cards must be included in the MCNP input file. The files card is used to specify an extra file that contains data for the MCNP-DSP calculations and to specify the output file for MCNP-DSP. The standard MCNP source and tally options are not available when using MCNP-DSP because the source and detector information are supplied in an extra data file. This chapter provides a listing of all of the options and their default values that can be used in the extra data file.

The following is a description of the options that can be supplied in the extra data file to execute MCNP-DSP calculations. The options are specified with a unique threecharacter identifier followed by an equal sign and an appropriate parameter. These options are similar to those found in the KENO-NR code and were chosen in this manner to minimize confusion when users switch from one code to the other.

The following options are required in each extra data file:

$\boldsymbol{s c d}=\boldsymbol{x} \quad \mathrm{x}$ is the number of source disintegrations per block. The default value has been arbitrarily chosen as 1 . A value of zero can be used if the inherent fission source option is used.

$\boldsymbol{b} \boldsymbol{k} \boldsymbol{s}=\boldsymbol{x} \quad \mathrm{x}$ is the number of blocks to calculate and must be an integer value greater than or equal to one. The default value has been arbitrarily chosen as 10000 .

$\boldsymbol{s c x}=\boldsymbol{x} \quad \mathrm{x}$ is the source $\mathrm{x}$-coordinate. There is no default variable for this parameter. The $\mathrm{x}$-coordinate cannot coincide with a surface.

scy $=y \quad y$ is the source $y$-coordinate. There is no default variable for this parameter. The y-coordinate cannot coincide with a surface.

$s c z=z \quad z$ is the source $\mathrm{z}$-coordinate. There is no default variable for this parameter. The z-coordinate cannot coincide with a surface.

$\boldsymbol{s c c}=\boldsymbol{x} \quad \mathrm{x}$ is the source cell and must be an integer value. If the source cell is not specified, it will be determined using the source coordinates.

$\boldsymbol{t} \boldsymbol{b n}=\boldsymbol{x}_{1} \boldsymbol{x}_{2} \quad \mathrm{x}_{1}$ is the number of time bins and must be a power of 2 no less than one and not greater than 2048.

$\mathrm{x}_{2}$ is the sampling rate and must be an integer greater than zero.

$\boldsymbol{d e t}=\boldsymbol{x}_{1 . .} \boldsymbol{x}_{13}$ The entries for the detectors depend on the detector type and the calculation mode. The first three entries on the detector 
card are the same regardless of detector type or calculation mode.

$\mathrm{x}_{1} \quad$ is the detector number. This entry must be an integer that begins with 2 and is labeled sequentially up to 5 .

$\mathrm{x}_{2} \quad$ is the detector material. This entry must be also be an integer and coincide with a material in the input file.

$\mathrm{x}_{3}$ is the detector type. The following are valid detector types:

$\mathrm{x}_{3}=2$ for capture detectors;

$\mathrm{x}_{3}=3$ for scattering detectors; and

$\mathrm{x}_{3}=4$ for fission detectors.

\section{CAPTURE DETECTORS (type 2)}

NEUTRON ONLY (MODE N)

$\mathrm{X}_{4} \quad$ is the number of random neutron counts per data block.

$\mathrm{X}_{5}$ is the detector efficiency. This parameter is used to account for thresholds set when using ${ }^{3} \mathrm{He}$ detectors.

$\mathrm{x}_{6} \quad$ is the detector dead time in nanoseconds.

NEUTRON AND PHOTONS (MODE N P)

$\mathrm{X}_{4} \quad$ is the lower photon energy threshold in $\mathrm{MeV}$ and must be greater than or equal to zero.

$\mathrm{x}_{5} \quad$ is the upper photon energy threshold in $\mathrm{MeV}$ and must be greater than the lower energy threshold

$\mathrm{x}_{6}$ is the pulse generation time entered in nanoseconds and must be greater than or equal to zero.

$\mathrm{x}_{7} \quad$ is the number of random neutron counts per data block.

$\mathrm{x}_{8} \quad$ is the number of random photon counts per data block.

$\mathrm{X}_{9} \quad$ is the detector efficiency. This parameter is used to account for thresholds set when using ${ }^{3} \mathrm{He}$ detectors.

$\mathrm{x}_{10} \quad$ is the detector dead time in nanoseconds.

\section{PHOTON ONLY (MODE P)}

$\mathrm{X}_{4} \quad$ is the lower photon energy threshold in $\mathrm{MeV}$ and must be greater than or equal to zero.

$\mathrm{x}_{5} \quad$ is the upper photon energy threshold in $\mathrm{MeV}$ and must be greater than the lower energy threshold

$\mathrm{x}_{6} \quad$ is the pulse generation time entered in nanoseconds and must be greater than or equal to zero.

$\mathrm{x}_{7} \quad$ is the number of random photon counts per data block.

$\mathrm{x}_{8} \quad$ is the detector efficiency. This parameter is used to account for thresholds set when using ${ }^{3} \mathrm{He}$ detectors.

$\mathrm{x}_{9} \quad$ is the detector dead time in nanoseconds. 
SCATTER DETECTORS (type 3)

NEUTRON ONLY (mode $n$ )

$\mathrm{x}_{4} \quad$ is the lower neutron energy threshold in $\mathrm{MeV}$ and must be greater than or equal to zero.

$\mathrm{X}_{5}$ is the upper neutron energy threshold in $\mathrm{MeV}$ and must be greater than the lower energy threshold

$\mathrm{x}_{6} \quad$ is the pulse generation time entered in nanoseconds and must be greater than or equal to zero.

$\mathrm{x}_{7} \quad$ is the number of energy bins to bin the detector responses.

$\mathrm{x}_{8} \quad$ is the number of random neutron counts per data block.

$\mathrm{X}_{9} \quad$ is the detector dead time in nanoseconds

PHOTON ONLY (mode p)

$\mathrm{x}_{4} \quad$ is the lower photon energy threshold in $\mathrm{MeV}$ and must be greater than or equal to zero.

$\mathrm{x}_{5} \quad$ is the upper photon energy threshold in $\mathrm{MeV}$ and must be greater than the lower energy threshold

$\mathrm{x}_{6} \quad$ is the pulse generation time entered in nanoseconds and must be greater than or equal to zero.

$\mathrm{x}_{7} \quad$ is the number of energy bins to bin the detector responses.

$\mathrm{x}_{8} \quad$ is the number of random photon counts per data block.

$\mathrm{x}_{9} \quad$ is the detector dead time in nanoseconds

NEUTRON AND PHOTON (mode $n \mathrm{p}$ )

$\mathrm{x}_{4} \quad$ is the lower neutron energy threshold in $\mathrm{MeV}$ and must be greater than or equal to zero.

$\mathrm{x}_{5}$ is the upper neutron energy threshold in $\mathrm{MeV}$ and must be greater than the lower energy threshold

$\mathrm{x}_{6} \quad$ is the lower photon energy threshold in $\mathrm{MeV}$ and must be greater than or equal to zero.

$\mathrm{x}_{7} \quad$ is the upper photon energy threshold in $\mathrm{MeV}$ and must be greater than the lower energy threshold

$\mathrm{x}_{8} \quad$ is the pulse generation time entered in nanoseconds and must be greater than or equal to zero.

$\mathrm{x}_{9} \quad$ is the number of energy bins to bin the detector responses.

$\mathrm{x}_{10}$ is the number of random neutron counts per data block.

$\mathrm{x}_{11}$ is the number of random photon counts per data block.

$\mathrm{x}_{12}$ is the detector dead time in nanoseconds. 
FISSION DETECTORS (type 4)

NEUTRON OR NEUTRON AND PHOTON (mode $\mathrm{n}$ or mode $\mathrm{n}$ )

$\mathrm{x}_{4} \quad$ is the number of random neutron counts per data block.

$\mathrm{x}_{5} \quad$ is the detector dead time in nanoseconds.

\section{SHOULD NOT BE USED FOR PHOTON ONLY (MODE P)}

At least two and no greater than four detectors must be specified for frequency analysis or correlation domain calculations.

At least one and no greater than four detectors specified for pulsed neutron calculations.

The following options are additional options that can be used in the calculations.

$\boldsymbol{s n u}=x_{1} \ldots x_{10}$ This parameter allows the user to change the default neutron emission distribution for the ${ }^{252} \mathrm{Cf}$ source. $\mathrm{x}_{1}$ is the probability of 0 neutrons from ${ }^{252} \mathrm{Cf}, \mathrm{x}_{2}$ is the probability of 1 neutron from ${ }^{252} \mathrm{Cf}, \mathrm{x}_{3}$ is the probability of 2 neutrons from ${ }^{252} \mathrm{Cf}$, and so forth, with a maximum of 9 neutrons from ${ }^{252} \mathrm{Cf}$.

$s p u=x_{1} \ldots x_{29}$ This parameter allows the user to change the default photon emission distribution for the ${ }^{252} \mathrm{Cf}$ source. $\mathrm{x}_{1}$ is the probability of 0 photons from ${ }^{252} \mathrm{Cf}, \mathrm{x}_{2}$ is the probability of 1 photon from ${ }^{252} \mathrm{Cf}, \mathrm{x}_{3}$ is the probability of 2 photons from ${ }^{252} \mathrm{Cf}$, and so forth, with a maximum of 28 photons from ${ }^{252} \mathrm{Cf}$.

$\mathbf{c n g}=\mathbf{x} \quad$ This is the angular distribution option for the ${ }^{252} \mathrm{Cf}$ source.

If $\mathrm{x}=0$, an isotropic distribution for neutrons from fission is used.

If $\mathrm{x}=1$, the angular distribution for neutrons from fission is obtained from the Budtz-Jorgensen and Knitter data. $\mathrm{x}$ must be equal to zero or one.

ang $=\mathbf{x} \quad$ This is the angular distribution option for neutron from fission.

If $x=0$, the default distribution for neutrons from fission obtained from cross-section data files. If $\mathrm{x}=1$, the angular distribution for neutrons from fission obtained from Budtz-Jorgensen and Knitter data $\mathrm{x}$ must be equal to zero or one.

$s t p=x \quad$ This is the source type option. If $\mathrm{x}=0, \mathrm{a}{ }^{252} \mathrm{Cf}$ source is used while a D-T source is used if $x=1$ and a D-D source is used if $x=2$. The user can specify a generalized mono-energetic source with $x=3$. $x$ must be set equal to either zero, one, two, or three and is equal to zero by default. 
If $\mathrm{stp}=1$ or $\mathrm{stp}=2$ the source is a neutron only source with neutron energy of 14.1 or $2.45 \mathrm{MeV}$. If $\mathrm{stp}=1, \mathrm{stp}=2$, or $\mathrm{stp}=3$ then additional input items are required as follows.

$\mathrm{x}_{1}$ is the source mode. If $\mathrm{x}_{1}=0$, the source is a continuous source, and if $\mathrm{x}_{1}=1$ the source is a pulsed source. If $\mathrm{x}_{1}=1$ the following two inputs are required:

$\mathrm{x}_{11} \quad$ is the repetition rate of the pulsed source in $\mathrm{Hz}$.

$\mathrm{x}_{12}$ is the pulse width for the neutron pulse in nanoseconds. This allows for the neutrons from the source to be emitted over some time distribution. This value must be greater than or equal to zero.

$\mathrm{x}_{2} \quad$ is the type of source. The allowable selections are zero and one. If $\mathrm{x}_{2}$ is zero, then the neutrons are emitted isotropically from the point of origin specified by scx, scy, and scz. If $\mathrm{x}_{2}$ is equal to one, then the source has a directional dependence that can be specified using a reference vector and half of the solid angle for the neutrons. The following additional parameters are required if $\mathrm{x}_{2}=1$ :

$\mathrm{x}_{21} \quad$ is the cosine of the reference vector in the $\mathrm{x}$-direction.

$\mathrm{x}_{22}$ is the cosine of the reference vector in the y-direction.

$\mathrm{x}_{23}$ is the cosine of the reference vector in the $\mathrm{z}$ direction.

$\mathrm{x}_{24} \quad$ is half of the angle for neutron emission in degrees.

$\mathrm{x}_{\mathrm{o}}, \mathrm{y}_{\mathrm{o}}, \mathrm{z}_{\mathrm{o}}$

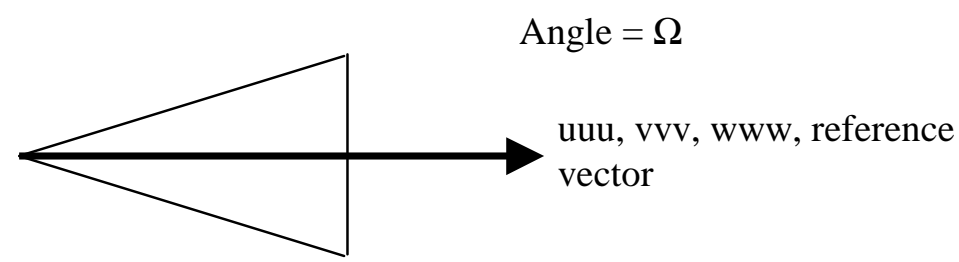


If $\operatorname{stp}=3$, then two additional input items are required.

$\mathrm{x}_{3}$ is the mono-energetic particle source type. $\mathrm{x}_{3}=1$ for $\mathrm{a}$ neutron source, or $\mathrm{x}_{3}=2$ for a photon source.

$\mathrm{x}_{4} \quad$ is the energy of the mono-energetic source.

fil $=x$

$\operatorname{win}=x$

$\operatorname{smo}=x$

$r m f=x$

$b d r=x$

ltm $=x$

pro $=x$
This is the parameter used to invoke the filter functions. If $x=0$, the filter functions are not used. If $x=1$, a Rockland filter is used in the calculations, and if $x=2$, a Precision filter is used. The default is no filtering. This option is only valid for frequency domain calculations.

This is the parameter to use the window functions. If $x=0$, the window functions are not used. A Bartlett window corresponds to $\mathrm{x}$ $=1$, a Hanning window corresponds to $\mathrm{x}=2$, and a Hamming window corresponds to $x=3$. The default is no windowing. This option is only valid for frequency domain calculations.

This option allows the user to Hanning window the frequency spectra to smooth the frequency data. The choices for $\mathrm{x}$ are 'yes' and 'no,' with the default being 'no.' This option is valid only for frequency domain calculations.

This option allows the user to remove the mean value from the detector time response prior to calculation of the frequency spectra or the correlation calculations. The choices for x are 'yes' and 'no,' with the default being 'no.'

This option is used to treat the detector response as a binary signal. Only a one or a zero is allowed in the detector time bins and extra counts are ignored. This option should only be used for sampling rates near $1 \mathrm{GHz}$. The binary detector option is invoked if $\mathrm{x}=1$ and is ignored otherwise. This option is only valid for correlation domain calculations.

This is the option used to perform the pulsed neutron calculations. The choices for $\mathrm{x}$ are 'yes' and 'no,' with 'no' being the default.

This option sets the processing mode for either frequency domain or correlation domain analyses. If $\mathrm{x}=0$, the standard frequency domain calculation is performed, and if $\mathrm{x}=1$, the correlation domain calculation is performed. If $\mathrm{x}=2$, then only the Feynman variance calculation is performed. 
This option allows the user to use the shift register technique to calculate the correlation functions. This method may be faster for computing correlation functions for a few select problems. This option is invoked if $x=1$ and is ignored otherwise. The default value for $\mathrm{x}$ is 0 .

$\boldsymbol{m l t}=\boldsymbol{x} \quad$ This is the multiplicity option. This option is invoked if $\mathrm{x}=1$ or $\mathrm{x}=2$ and is ignored otherwise. If this option is used, an additional output file is created that contains integers that represent the number of times $0,1,2$, etc., counts occurred in the data block for all detectors triggered and random. If this option is invoked, no other modes of processing will be performed.

If $x=1$ then the multiplicity data is acquired using the detector signal as the trigger signal.

If $x=2$ then the multiplicity data is acquired using the source signal as the trigger signal. This requires that an active simulation be performed.

$\boldsymbol{s} \boldsymbol{s} \boldsymbol{s}=\boldsymbol{x}_{1} . . \boldsymbol{x}_{\boldsymbol{j}} \quad$ The inherent spontaneous fission volume source allows the user to specify various inherent sources in up to 50 different volumes in the calculation. The user can specify up to 6 different spontaneous fission sources in each volume. This is useful for simulating spent fuel assemblies that contain multiple spontaneous fission sources such as ${ }^{242} \mathrm{Cm}$ and ${ }^{244} \mathrm{Cm}$.

$\mathrm{x}_{1} \quad$ is the total number of inherent fission sources for the problem.

$\mathrm{x}_{2}$ is the number of inherent source volumes.

$\mathrm{x}_{3}$ is the spontaneous fission source volume type. The number of entries to describe this volume depends on the volume type. The following are valid geometry types:

$\mathrm{x}_{3}=0$ : volume bounded by six planes.

$\mathrm{x}_{0} \quad \mathrm{x}$-coordinate of volume

$\mathrm{y}_{0} \quad \mathrm{y}$-coordinate of volume

$\mathrm{z}_{0} \quad \mathrm{z}$-coordinate of volume

$\mathrm{x}_{\mathrm{p}} \quad$ distance between $\mathrm{x}_{0}$ and positive $\mathrm{x}$-plane

$\mathrm{x}_{\mathrm{m}} \quad$ distance between $\mathrm{x}_{0}$ and negative $\mathrm{x}$-plane

$\mathrm{y}_{\mathrm{p}} \quad$ distance between $\mathrm{y}_{0}$ and positive $\mathrm{y}$-plane

$\mathrm{y}_{\mathrm{m}} \quad$ distance between $\mathrm{y}_{0}$ and negative $\mathrm{y}$-plane

$\mathrm{z}_{\mathrm{p}} \quad$ distance between $\mathrm{z}_{0}$ and positive $\mathrm{z}$-plane

$\mathrm{z}_{\mathrm{m}} \quad$ distance between $\mathrm{z}_{0}$ and negative $\mathrm{z}$-plane 
$\mathrm{x}_{3}=1$ : volume is cylinder parallel to $\mathrm{x}$-axis

$\mathrm{x}_{0} \quad \mathrm{x}$-coordinate of volume

$\mathrm{y}_{0} \quad \mathrm{y}$-coordinate of volume

$\mathrm{z}_{0} \quad \mathrm{z}$-coordinate of volume

$r_{i} \quad$ inner radius of cylinder

$r_{o} \quad$ outer radius of cylinder

$\mathrm{x}_{\mathrm{p}} \quad$ distance between $\mathrm{x}_{0}$ and positive $\mathrm{x}$-plane

$\mathrm{x}_{\mathrm{m}} \quad$ distance between $\mathrm{x}_{0}$ and negative $\mathrm{x}$-plane

$\mathrm{x}_{3}=2$ : volume is cylinder parallel to $\mathrm{y}$-axis

$\mathrm{x}_{0} \quad \mathrm{x}$-coordinate of volume

$\mathrm{y}_{0} \quad \mathrm{y}$-coordinate of volume

$\mathrm{z}_{0} \quad \mathrm{z}$-coordinate of volume

$r_{i} \quad$ inner radius of cylinder

$r_{o} \quad$ outer radius of cylinder

$\mathrm{y}_{\mathrm{p}} \quad$ distance between $\mathrm{y}_{0}$ and positive $\mathrm{y}$-plane

$\mathrm{y}_{\mathrm{m}} \quad$ distance between $\mathrm{y}_{0}$ and negative $\mathrm{y}$-plane

$\mathrm{x}_{3}=3$ : volume is cylinder parallel to $\mathrm{z}$-axis

$\mathrm{x}_{0} \quad \mathrm{x}$-coordinate of volume

$\mathrm{y}_{0} \quad \mathrm{y}$-coordinate of volume

$\mathrm{z}_{0} \quad \mathrm{z}$-coordinate of volume

$r_{i} \quad$ inner radius of cylinder

$r_{0} \quad$ outer radius of cylinder

$\mathrm{z}_{\mathrm{p}} \quad$ distance between $\mathrm{z}_{0}$ and positive $\mathrm{z}$-plane

$\mathrm{z}_{\mathrm{m}} \quad$ distance between $\mathrm{z}_{0}$ and negative $\mathrm{z}$-plane

$\mathrm{x}_{3}=4$ : volume is sphere
$\mathrm{x}_{0} \quad \mathrm{x}$-coordinate of volume
$\mathrm{y}_{0} \quad \mathrm{y}$-coordinate of volume
$\mathrm{z}_{0} \quad \mathrm{Z}$-coordinate of volume
$r_{i} \quad$ inner radius of sphere
$r_{o} \quad$ outer radius of sphere

Entry 3 along with the appropriate geometry variables are provided for each spontaneous fission source volume specified by $\mathrm{x}_{2}$.

$\mathrm{x}_{4} \quad$ is the number of inherent spontaneous fission sources for volume $\mathrm{i}$.

$\mathrm{x}_{5} \quad$ is spontaneous fission source type $\mathrm{j}$ for volume $\mathrm{i}$.

$$
\begin{aligned}
& =1 \quad{ }^{238} \mathrm{U} \\
& =2 \quad{ }^{240} \mathrm{Pu} \\
& =3 \quad{ }^{242} \mathrm{Pu} \\
& =4 \quad{ }^{242} \mathrm{Cm} \\
& =5 \quad{ }^{244} \mathrm{Cm} \\
& =6 \quad{ }^{238} \mathrm{Pu}
\end{aligned}
$$


$\mathrm{x}_{6} \quad$ is the number of spontaneous fissions per block for isotope $\mathrm{j}$ in volume $\mathrm{i}$.

Entries 5 and 6 are made for each spontaneous fission source for volume i as Specified by $\mathrm{x}_{4}$.

$\boldsymbol{c n m}=\boldsymbol{x} \quad$ This option is used to normalize the correlation functions by dividing the correlation functions by the number of source events and multiplying by the number of time bins in the simulation.

If $x=1$, the correlation functions are normalized; otherwise, if $x=0$ (default) then the correlation functions are not normalized.

$z \boldsymbol{f n}=\boldsymbol{x} \quad$ This option is used to allow zero neutrons from fission. If $\mathrm{x}=1$, then no neutrons are emitted from fission; otherwise, if $\mathrm{x}=0$ (default) then the number of fission neutrons is sampled from the appropriate distribution.

$\boldsymbol{s t m}=\boldsymbol{x} \quad$ This option is used in the frequency or correlation analysis simulations to force the source starting time to be zero. If $x=1$, the source starts at time zero; otherwise, if $x=0$ (default) the source starting time is random for active frequency or correlation simulations.

$\boldsymbol{f n u}=\boldsymbol{x} \quad$ This option is used to control the how the fission neutron distribution data are used. Originally, the fission neutron distribution data of Zucker and Holden was fit as a function of energy to determine the number of neutrons from fission. The data at thermal energies was then modified to coincide with the data of Gwin et al. to provide a second option. The distributions differ in the fact that the Gwin et al. data has a value of 2.437 for the average number of neutrons from thermal neutron induced fission of ${ }^{235} \mathrm{U}$ whereas the Zucker and Holden data has a value of 2.417. The latest addition involved fitting the Zucker and Holden distribution data as a function of the average number of neutrons from fission to obtain corresponding probability distributions. This latest addition is the recommended option because the probability distributions are dependent on the cross section data set because the average number of neutrons from fission depends on the cross section data set.

If $x=1$, then the normalized probability distribution of Gwin et al. is used for the thermal neutron induced fission of ${ }^{235} \mathrm{U}$. If $\mathrm{x}=0$, then the normalized probability distribution of Zucker and Holden is used for the thermal neutron induced fission of ${ }^{235} U$. If $x=2$ (default), then the distribution is determined based on the average number of neutrons from fission that is energy dependent. 
$\boldsymbol{c n s}=\boldsymbol{x} \quad$ This option is used to specify the prompt neutron fission spectrum for spontaneous fission of ${ }^{252} \mathrm{Cf}$.

If $\mathrm{x}=0$ (default), then the Mannhart corrected Maxwellian spectrum is used as the energy spectrum for prompt neutrons from the spontaneous fission of ${ }^{252} \mathrm{Cf}$.

If $x=1$, then the Madland-Nix theoretical spectrum is used.

If $x=2$, then the Froehner Watt spectrum is used.

$\boldsymbol{c n u} \boldsymbol{u} \boldsymbol{x} \quad$ This option is used to specify the prompt neutron emission probability distribution for the spontaneous fission of ${ }^{252} \mathrm{Cf}$. The options are either to use the Spencer et al. data or to use the Boldeman data. The average number of neutrons from Spencer's distribution is 3.773 whereas the average number of neutrons from Boldeman's data is 3.757 .

If $x=0$ (default), then the distribution of Spencer et al. is used in the simulations whereas if $\mathrm{x}=1$, the distribution of Boldeman is used in the simulations.

$\boldsymbol{d t o}=\boldsymbol{x} \quad$ This option is used to specify the dead time option.

If $x=0$ then the dead time option is disabled.

If $x=1$ (default), a non-paralyzable dead time model is used that eliminates counts that occur after a pulse within the dead time.

If $\mathrm{x}=2$, a paralyzable dead time model is used that eliminates counts that occur after a pulse and extends the dead time period.

$\boldsymbol{s} \boldsymbol{d} \boldsymbol{t}=\boldsymbol{x} \quad$ This option is used to specify the dead time of the source for active measurements in nanoseconds. The user must supply the dead time in nanoseconds. This option should only be used for active simulations with a ${ }^{252} \mathrm{Cf}$ source. 


\section{SIMULATION EXAMPLES}

This chapter provides several examples for the multiple simulation modes that are available using the code. Models have been created to demonstrate the various source and detector options for several different calculation modes. Examples are provided for frequency analysis, correlation analysis, pulsed source, Feynman variance, and multiplicity simulations. Unless otherwise specified these are generic models that do not correspond to any actual measurement.

\subsection{FREQUENCY ANALYSIS SIMULATIONS}

\subsubsection{Active Simulations}

\subsubsection{Uranium Metal sphere with Lithium Glass Detectors}

The first example demonstrates a simulation with a ${ }^{252} \mathrm{Cf}$ source that is located within a highly enriched uranium metal sphere. The uranium metal density is $18.75 \mathrm{~g} / \mathrm{cm}^{3}$, and the uranium isotopic content is $93.15 \mathrm{wt} \%{ }^{235} \mathrm{U}, 5.64 \mathrm{wt} \%{ }^{238} \mathrm{U}, 0.97 \mathrm{wt} \%{ }^{234} \mathrm{U}$, and $0.24 \mathrm{wt} \%{ }^{236} \mathrm{U}$. Two lithium glass scintillation detectors (5.08-cm OD, 2.54-cm long) are located adjacent to the sphere and are positioned $180^{\circ}$ apart. The MCNP input file is given in Fig. 6.1, and the extra data file contents for the frequency domain calculations are given in Fig. 6.2. Only an overview of the MCNP input file is presented.

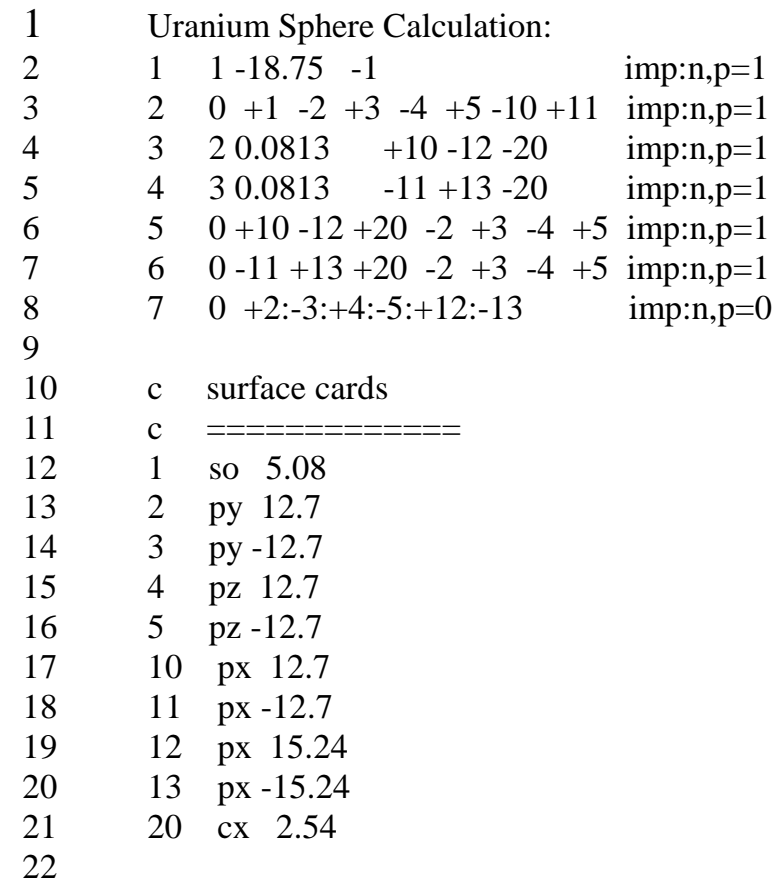

Figure 6.1. MCNP-DSP input file elala.i. 


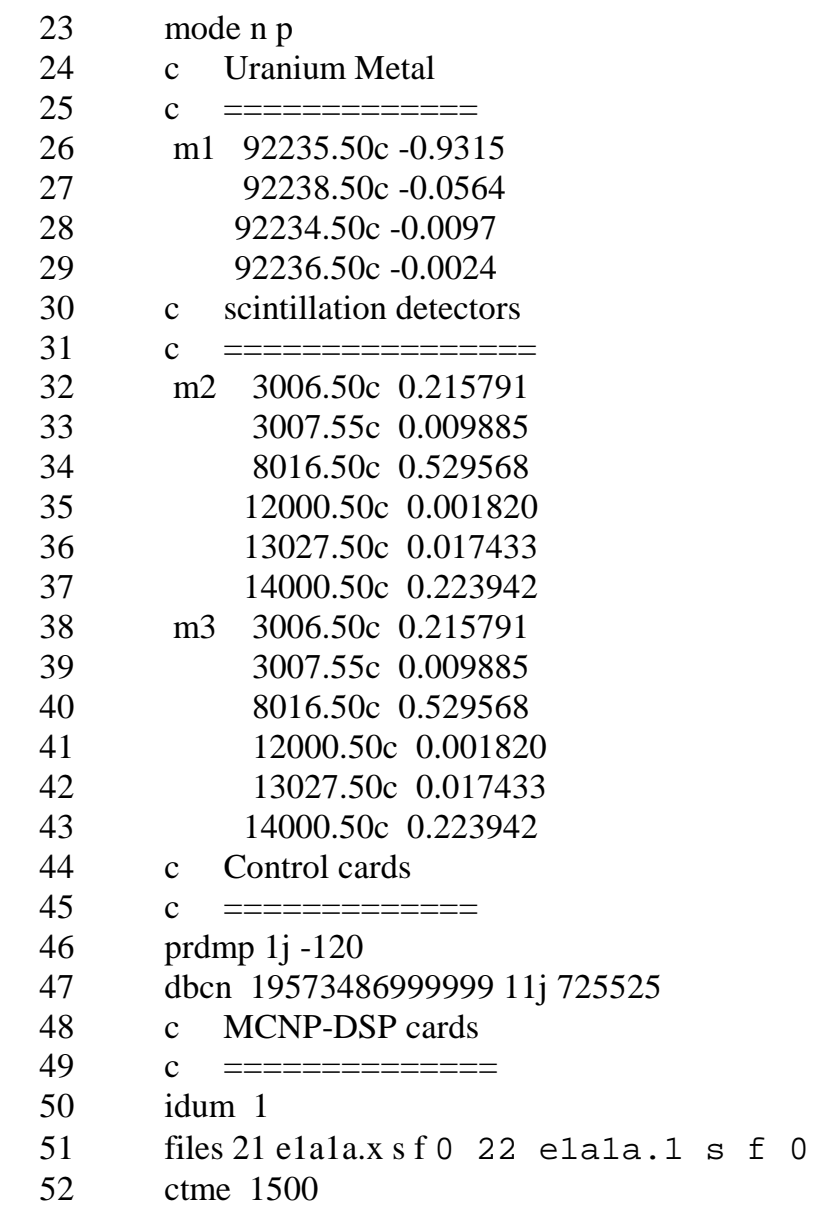

Figure 6.1. (continued).

\section{Description of Input File E1A1A.I}

Line 1: $\quad$ The first line of the input file is the title line.

Lines 2-8: $\quad$ These are the cell description cards. The cell cards are used to describe the composition of a cell and the surfaces that bound the particular cell. The cells must be numbered sequentially, and cells that are not used in the model must be removed. Otherwise, MCNP-DSP will produce errors in the calculations.

Lines 10-21: These lines contain the surface description cards. For this simple problem the surfaces consist of planes and cylinders. Note that the surface numbers do not have to be listed sequentially.

Lines 23-47: These are the data cards. Line 23 indicates that this is a dual neutron and photon calculation. Lines 24 through 43 describe the materials used in the calculations. The materials must be numbered sequentially. No 
materials can be skipped nor should unused materials be added to the input file. A separate material must be entered for each detector because the detector tallies are determined by the material in which interactions occur. Lines 44 through 47 are the control cards for this problem. The prdmp card on line 46 is used to control how often data are written to the MCNP-DSP output files. The $d b c n$ card on line 47 is used to change the random number seed for the MCNP-DSP because the output data for several MCNP-DSP calculations are typically combined together.

Lines 48-51: These lines are used to execute the MCNP-DSP calculations. If the first entry on the idum card is 1, the MCNP-DSP calculation is executed and other tallies are not accumulated. The files card is used to specify two files. The first file (e1a1a.x) contains the extra data parameters and is opened as a sequential, formatted file on unit 21. The second file (e1a1a.1) is the MCNP-DSP output file. The output file is opened as a sequential formatted file on unit 22. Both files must be entered as sequential and formatted files. The record length is zero for sequential files.

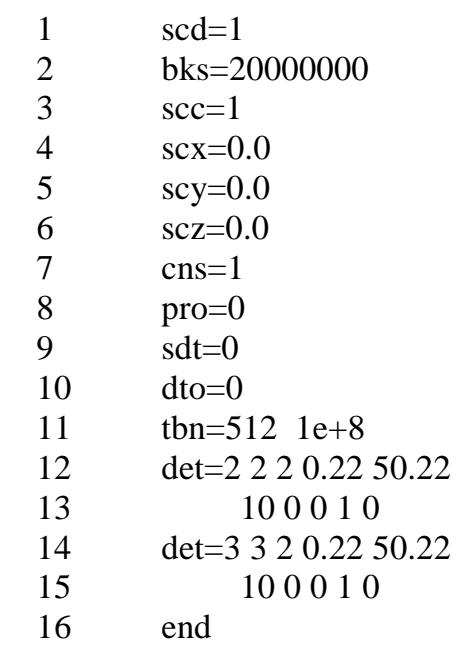

Figure 6.2. MCNP-DSP extra data file elala.x.

\section{Description of Extra Data File E1A1A.X}

Line 1: $\quad$ One source disintegration per block $(s c d)$ in this example.

Line 2: $\quad$ The number of blocks to calculate $(b k s)$ is 20000000.

Lines 3-6: These lines represent the coordinates of the source. Line 3 is the source cell while lines 4-6 correspond to the origin of the source. 
Line 7: $\quad$ This parameter specifies that the Madland-Nix prompt neutron spectrum is to be used in the simulation instead of the default Mannhart corrected Maxwellian spectrum.

Line 8: This line specifies that this is a frequency analysis calculation.

Line 9: $\quad$ This specifies that the source dead time is zero nanoseconds.

Line 10: This line specifies that the dead time option be disabled.

Line 11: This line specifies the number of time channels as 512 with a sampling rate of $100 \mathrm{MHz}$.

Lines 12-15: These lines describe the detectors in the problem. The source is detector 1 and does not need to be labeled. This problem has two detectors, 2 and 3. Both detectors are capture detectors. Detector 2 is material 2 and type 2 while detector 3 is material 3 and type 2 . The gamma ray thresholds must be provided because this is a dual neutron and photon simulation and the lithium glass detector response is affected by gamma rays. The lower and upper gamma ray thresholds are 0.22 and $50.22 \mathrm{MeV}$ respectively. The pulse generation time is set to $10 \mathrm{~ns}$. There are no neutron or gamma ray background counts for the detector. The neutron capture efficiency is 1 for each detector, and the dead time for each detector is zero. For the frequency domain calculations, at least two detectors ( 2 and 3 ) must be listed in the extra data file.

Line 16: The end parameter is used to specify the end of the extra file. This option could have also been omitted.

Two or more output files are created when performing frequency domain calculations. The first contains general information on the number of counts for each detector and is specified as the second data file in the MCNP input file. The other output file(s) contains the frequency spectra for the detectors. If the problem is a combined neutron-photon calculation as in the example, there will be three files created that contain the auto and cross spectra for the detectors for the neutron response, the gamma response, and the combined neutron and gamma ray response. The general output filename as specified in Fig. 6.1 is elala.1. Three additional files will contain the auto and cross spectra. For a computer with the Unix operating system, these are elala.1.123.fft.n, elala.1.123.fft.p, and elala.1.123.fft.np, where the first file contains the spectra for the neutron response, the second file contains the spectra for the gamma response, and the third file contains the spectra for the combined neutron and gamma ray response as indicated by the last extension. The other filename extensions have distinct meanings. The first part that has the ' 123 ' indicates that this file contains the frequency spectra for the auto spectra of detectors \#1, \#2, and \#3, and the cross spectra between 1 and 2, 1 and 3 , and 2 and 3. The ' $f f t$ ' indicates that the file contains data that have been Fourier transformed. Comments concerning the output file names are provided in Appendix D. 
The frequency spectra for multiple runs can be combined by combining the auto and cross spectra based on the number of blocks of data accumulated. If the user combines the frequency spectra from several runs, the Monte Carlo calculation for each run must begin with a different random number seed that can be specified using the $d b c n$ card in the MCNP input file.

\section{Description of Output Summary File E1A1A.1}

The MCNP-DSP output summary file (elala.1) is provided in Fig. 6.3 and begins with a description of the source used for the calculations. The average number of source events per data block and the number of data blocks accumulated are printed. The source cell and coordinate positions are also given. Next, the detector options used in the calculation are given. After the detector options are listed, the neutron tracking information is provided for all neutrons and for the source neutrons. The number of fissions, captures, and $(\mathrm{n}, \mathrm{xn})$ reactions and the number of leakages are tallied for all neutrons and tallied separately for source neutrons. The average number of neutrons from the source and system fissions are estimated along with the Diven factor for both source and system neutrons. The average energy of neutrons from the source fission and from system fissions is also calculated. A probability distribution for the number of neutrons from the source and the system is then provided. After these parameters, the total number of neutron detections is given for the number of blocks specified at the beginning of the output file. The number of detections due to directly counting source particles is also listed. Information similar to that for the neutrons is also provided for the gamma rays.

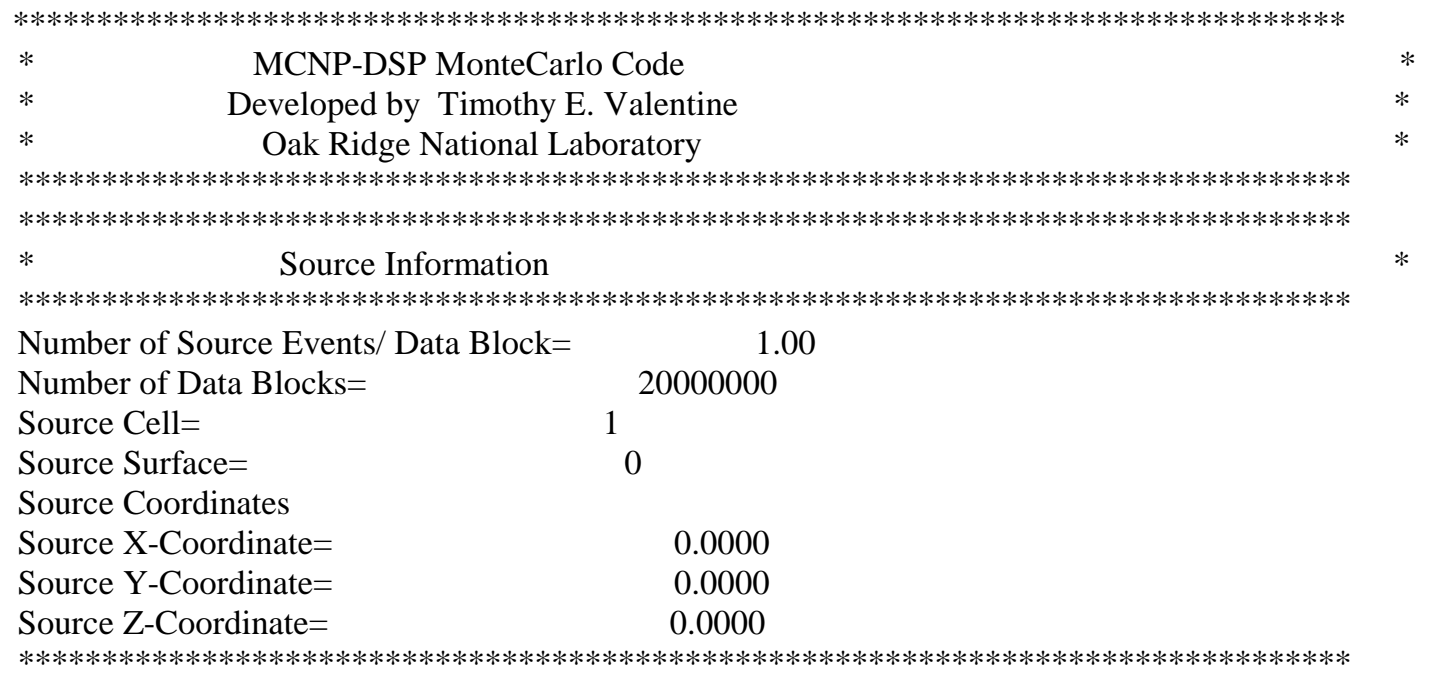

Figure 6.3. MCNP-DSP output summary file e1a1a.1. 


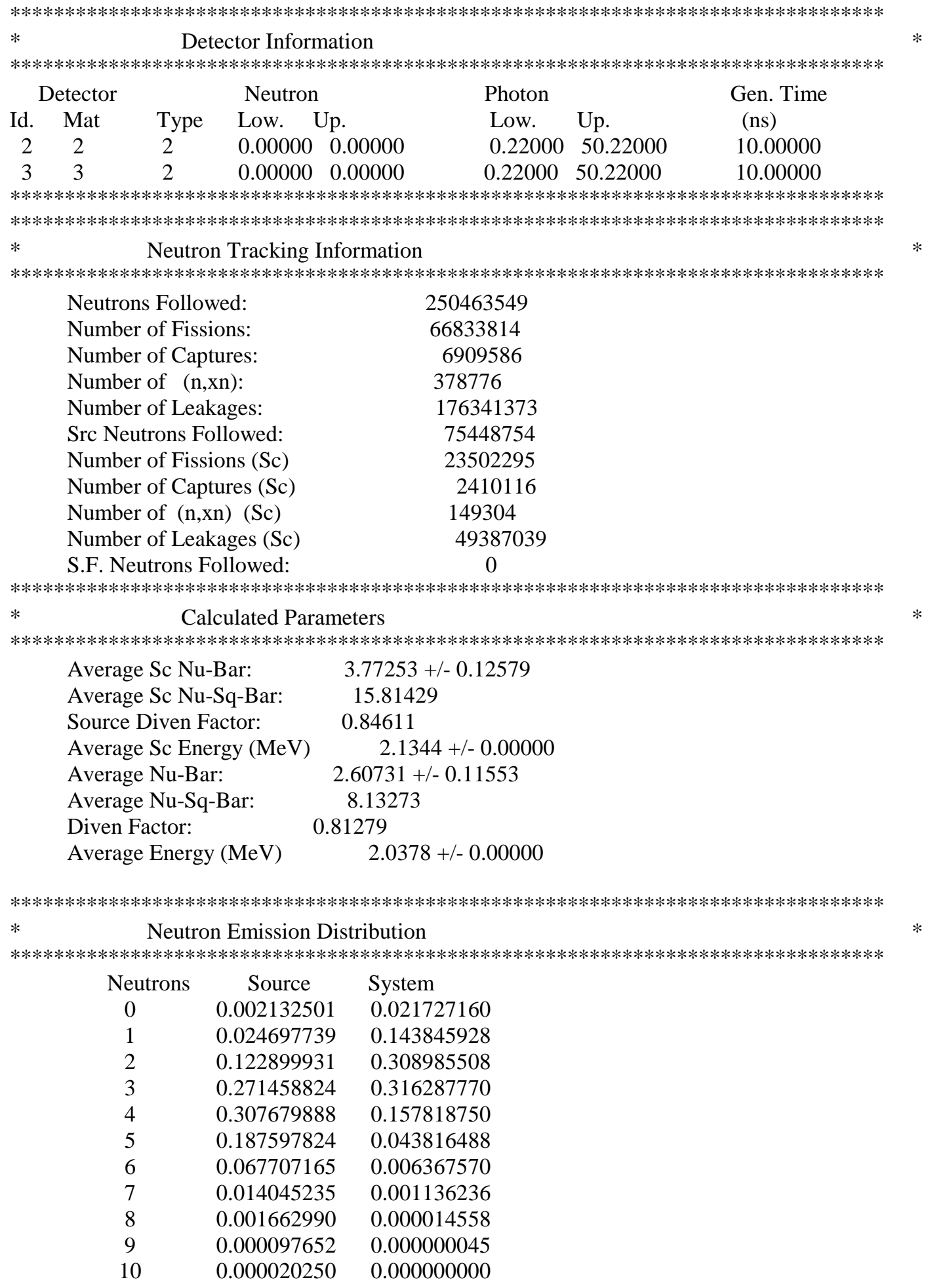

Figure 6.3. (continued). 


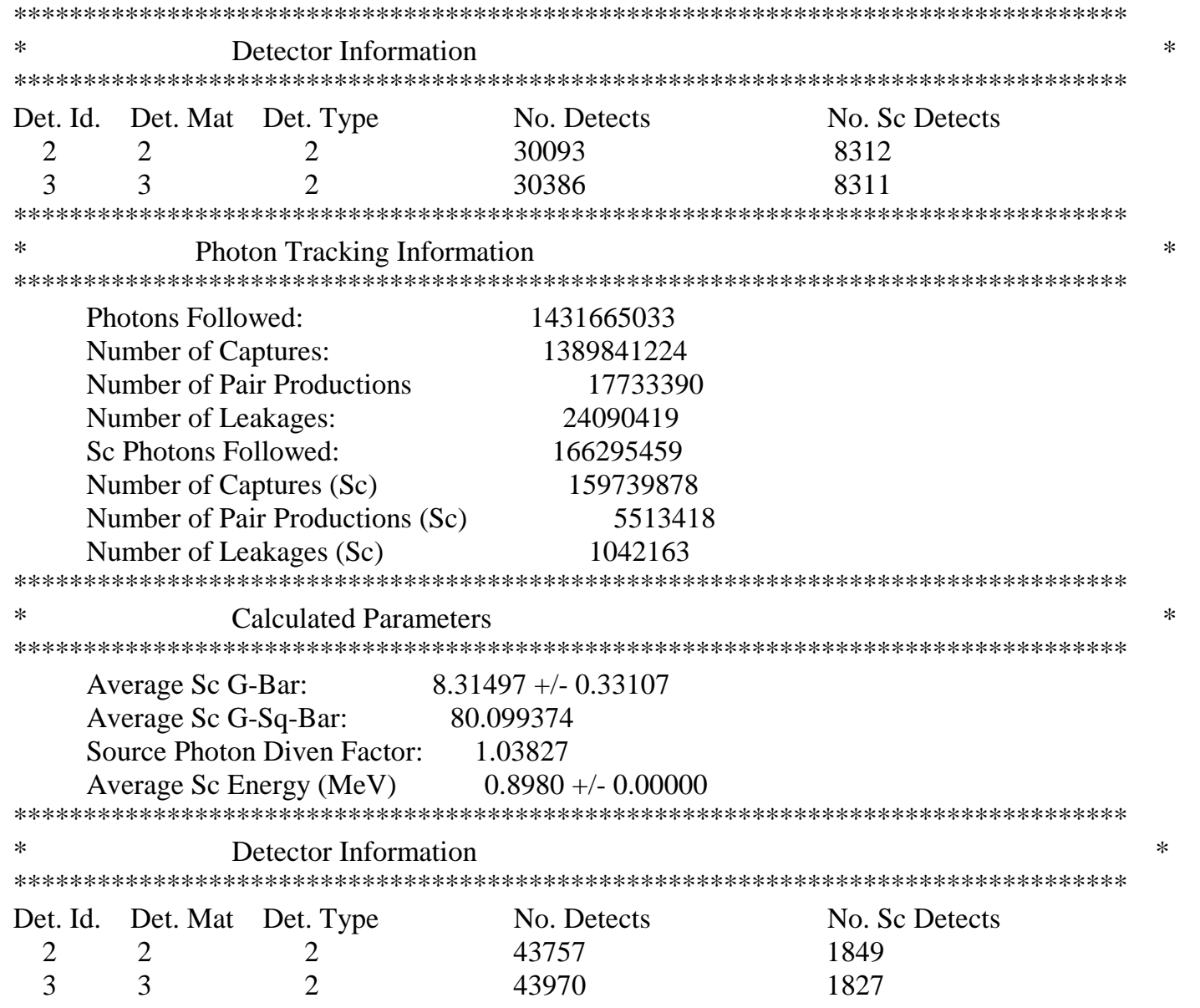

Figure 6.3. (continued).

\section{Description of Output Summary File E1A1A.1.123.FFT.N}

Part of the MCNP-DSP output file elala.1.123.fft.n is provided in Fig. 6.4. This output file contains the frequency dependent auto and cross spectra for the neutron detections. The output file contains the frequency interval, the Nyquist frequency, the number of frequency points, and the number of data blocks that were accumulated during the calculation. The other entries in this file is as follows: auto spectra of the source, auto spectra of the detectors, the real part of the cross spectra between the source and detector 2 , the imaginary part of the cross spectra between the source and detector 2, the real part of the cross spectra between the source and detector 3 , the imaginary part of the cross spectra between the source and detector 3, the real part of the cross spectra between detectors, and the imaginary part of the cross spectra between detectors. The data in this file can be used to calculate the coherence between detectors or the spectral ratio using appropriate software. Similar output files are created for the photon response and the combined neutron and photon response. 


\begin{tabular}{|c|c|c|c|c|c|c|c|c|c|}
\hline $\begin{array}{l}\text { Delta F } \\
1.95312 \mathrm{E}+05\end{array}$ & $\begin{array}{l}\text { Nyquist } \\
\text { 5.00000E+07 }\end{array}$ & $\begin{array}{c}\text { Blocks } \\
2.0000\end{array}$ & 0E+07 & & & & & & \\
\hline Frequency & PSD 11 & PSD22 & PSD33 & Re G12 & $\operatorname{Im}$ G12 & Re G13 & Im G13 & $\operatorname{Re} \mathrm{G} 23$ & Im G23 \\
\hline $0.00000000 \mathrm{E}+00$ & $1.99993965 \mathrm{E}+00$ & $1.51175000 \mathrm{E}-03$ & $1.52650000 \mathrm{E}-03$ & $3.17720000 \mathrm{E}-03$ & $0.00000000 \mathrm{E}+00$ & $3.18700000 \mathrm{E}-03$ & $0.00000000 \mathrm{E}+00$ & $6.40000000 \mathrm{E}-06$ & $5 \quad 0.00000000 \mathrm{E}+0$ \\
\hline $1.95312500 \mathrm{E}+05$ & $1.00001728 \mathrm{E}+00$ & $1.50907947 \mathrm{E}-03$ & 1.52409730E-03 & $1.51350353 \mathrm{E}-03$ & $-4.31148377 \mathrm{E}-05$ & $1.51538628 \mathrm{E}-03$ & $-3.71837179 \mathrm{E}-05$ & $3.76060959 \mathrm{E}-06$ & $4.34808619 \mathrm{E}-08$ \\
\hline $3.90625000 \mathrm{E}+05$ & $9.99929221 \mathrm{E}-01$ & 1.50891164E-03 & $1.52412400 \mathrm{E}-03$ & $1.50166115 \mathrm{E}-03$ & $-9.17308751 \mathrm{E}-05$ & $1.51672671 \mathrm{E}-03$ & $-7.64808156 \mathrm{E}-05$ & 3.38790615E-06 & 2.11548187E-07 \\
\hline $5.85937500 \mathrm{E}+05$ & $9.99965138 \mathrm{E}-01$ & $1.50924684 \mathrm{E}-03$ & & $1.49148390 \mathrm{E}-03$ & $-1.25865128 \mathrm{E}-04$ & & & & \\
\hline $7.81250000 \mathrm{E}+05$ & $9.99865320 \mathrm{E}-01$ & $1.50823667 \mathrm{E}-03$ & & & $-1.66741659 \mathrm{E}-04$ & & & & \\
\hline $9.76562500 \mathrm{E}+05$ & 9.99653854E-01 & $1.50937087 \mathrm{E}-03$ & $1.52410829 \mathrm{E}-03$ & $1.48273429 \mathrm{E}-03$ & $-2.04193791 \mathrm{E}-04$ & $1.51263672 \mathrm{E}-03$ & $-2.05016995 \mathrm{E}-04$ & 3.86080954E-06 & 5.58974603E-07 \\
\hline $1.17187500 \mathrm{E}+06$ & $1.00021580 \mathrm{E}+00$ & $1.50872406 \mathrm{E}-03$ & $1.52403486 \mathrm{E}-03$ & $1.47320492 \mathrm{E}-03$ & $-2.40924163 \mathrm{E}-04$ & $1.48627624 \mathrm{E}-03$ & $-2.45601029 \mathrm{E}-04$ & $3.58480816 \mathrm{E}-06$ & $1.84040929 \mathrm{E}-07$ \\
\hline $1.36718750 \mathrm{E}+06$ & $1.00002193 \mathrm{E}+00$ & & & & & & & & \\
\hline $1.75781250 \mathrm{E}+06$ & $1.00015047 \mathrm{E}+00$ & $1.50844621 \mathrm{E}-03$ & $1.52327391 \mathrm{E}-03$ & $1.43665924 \mathrm{E}-03$ & $-3.56397987 \mathrm{E}-04$ & $1.46244013 \mathrm{E}-03$ & $-3.74581614 \mathrm{E}-04$ & 3.27274353E-06 & 4.02002513E-07 \\
\hline $500 \mathrm{E}+06$ & $1.00043910 \mathrm{E}+00$ & $1.50839529 \mathrm{E}-03$ & $1.52341032 \mathrm{E}-03$ & E-03 & E-04 & & E-04 & 57E-06 & E-07 \\
\hline $2.14843750 \mathrm{E}+06$ & & & & & & & & & \\
\hline $2.34375000 \mathrm{E}+06$ & $1.00000423 \mathrm{E}+00$ & $1.50965559 \mathrm{E}-03$ & $1.52383630 \mathrm{E}-03$ & 1.38082492E-03 & $-4.69465201 \mathrm{E}-04$ & $1.40285122 \mathrm{E}-03$ & $-4.81990765 \mathrm{E}-04$ & 3.59121222E-06 & $3.36833218 \mathrm{E}-07$ \\
\hline $2.53906250 \mathrm{E}+06$ & 9.99908299E-01 & $1.50892774 \mathrm{E}-03$ & $1.52418507 \mathrm{E}-03$ & 1.37940814E-03 & $-5.04806660 \mathrm{E}-04$ & & & 3.27993664E-06 & $1.62343075 \mathrm{E}-07$ \\
\hline $0 \mathrm{E}+06$ & 9.995 & $54 \mathrm{E}-03$ & $1.52 ?$ & & & & & & -07 \\
\hline $2.92968750 \mathrm{E}+06$ & $1.00018315 \mathrm{E}+00$ & 50890442E-03 & & & & & & & -07 \\
\hline $3.12500000 \mathrm{E}+06$ & $1.00001031 \mathrm{E}+00$ & $1.50860362 \mathrm{E}-03$ & 7095E-03 & 379022E-03 & 792E-04 & $1.32226671 \mathrm{E}-03$ & $70 \mathrm{E}-04$ & 82E-06 & $8 \mathrm{E}-07$ \\
\hline $31250 \mathrm{E}+06$ & $615 \mathrm{E}+00$ & $1.50846249 \mathrm{E}-03$ & 1.52 & & & & & & 4E-07 \\
\hline $0 \mathrm{E}+06$ & 9.998 & $03 \mathrm{E}-03$ & & & & & & & -07 \\
\hline $5250 \mathrm{E}+06$ & $1.00002 \mathrm{C}$ & $1.50869332 \mathrm{E}-03$ & & & & & & & \\
\hline $00 \mathrm{E}+06$ & 9.99 & & & & & & & & \\
\hline $4.49218750 \mathrm{E}+06$ & 9.99896511E-01 & $1.50874008 \mathrm{E}-03$ & $1.52356028 \mathrm{E}-03$ & $1.14809535 \mathrm{E}-03$ & $-8.00849611 \mathrm{E}-04$ & $1.14593347 \mathrm{E}-03$ & $-8.30300016 \mathrm{E}-04$ & $2.76203985 \mathrm{E}-06$ & $3.50850257 \mathrm{E}-07$ \\
\hline $4.68750000 \mathrm{E}+06$ & $1.00002798 \mathrm{E}+00$ & $1.50825977 \mathrm{E}-03$ & & $1.11565881 \mathrm{E}-03$ & $27068 \mathrm{E}-04$ & $1.12083122 \mathrm{E}-03$ & $-8.62385705 \mathrm{E}-04$ & 2.82089567E-06 & 189E-07 \\
\hline & & & & & & & & & \\
\hline $2500 \mathrm{E}+06$ & $1.00026365 \mathrm{E}+00$ & & & & & & & & 9E-07 \\
\hline $5.27343750 \mathrm{E}+06$ & 9.99728377E-01 & $1.50853170 \mathrm{E}-03$ & $1.52336638 \mathrm{E}-03$ & $1.01953412 \mathrm{E}-03$ & $-8.88923149 \mathrm{E}-04$ & $1.03807923 \mathrm{E}-03$ & $-9.02617942 \mathrm{E}-04$ & $2.97674356 \mathrm{E}-06$ & $-4.93824686 \mathrm{E}-08$ \\
\hline $5.46875000 \mathrm{E}+06$ & $1.00000687 \mathrm{E}+00$ & $1.50860611 \mathrm{E}-03$ & $1.52360738 \mathrm{E}-03$ & $1.01781505 \mathrm{E}-03$ & $-9.17051543 \mathrm{E}-04$ & $1.01356283 \mathrm{E}-03$ & $-9.28591667 \mathrm{E}-04$ & $3.12892124 \mathrm{E}-06$ & $3.31376284 \mathrm{E}-07$ \\
\hline $5.66406250 \mathrm{E}+06$ & & & & & & & & $2.90277563 \mathrm{E}-06$ & $-2.78112395 \mathrm{E}-07$ \\
\hline $5.85937500 \mathrm{E}+06$ & $9.99812055 \mathrm{E}-01$ & $1.50857024 \mathrm{E}-03$ & $1.52317335 \mathrm{E}-03$ & $9.45357027 \mathrm{E}-04$ & $-9.51624020 \mathrm{E}-04$ & $9.32802458 \mathrm{E}-04$ & $-9.63252363 \mathrm{E}-04$ & 2.33324394E-06 & $2.76048583 \mathrm{E}-07$ \\
\hline $6.05468750 \mathrm{E}+06$ & $1.00021039 \mathrm{E}+00$ & $1.50847104 \mathrm{E}-03$ & $1.52262377 \mathrm{E}-03$ & $9.06878509 \mathrm{E}-04$ & $-9.65544629 \mathrm{E}-04$ & $9.15865928 \mathrm{E}-04$ & $-9.79429756 \mathrm{E}-04$ & 3.09552502E-06 & $2.04319768 \mathrm{E}-07$ \\
\hline $6.25000000 \mathrm{E}+06$ & $1.00014359 \mathrm{E}+00$ & $1.50850533 \mathrm{E}-03$ & $1.52334929 \mathrm{E}-03$ & 8.74280911E-04 & $-9.78077537 \mathrm{E}-04$ & 8.85795941E-04 & $-9.97921843 \mathrm{E}-04$ & $3.15311871 \mathrm{E}-06$ & $3.38584622 \mathrm{E}-08$ \\
\hline
\end{tabular}

Figure 6.4. MCNP-DSP output file e1a1a.1.123.fft.n 


\subsubsection{Uranium Metal Sphere with Plastic Scintillation Detectors}

This example demonstrates a simulation with a ${ }^{252} \mathrm{Cf}$ source that is located within a highly enriched uranium metal sphere. Again, the uranium metal density is $18.75 \mathrm{~g} / \mathrm{cm}^{3}$, and the uranium isotopic content is $93.15 \mathrm{wt} \%{ }^{235} \mathrm{U}, 5.64 \mathrm{wt} \%{ }^{238} \mathrm{U}, 0.97 \mathrm{wt} \%{ }^{234} \mathrm{U}$, and $0.24 \mathrm{wt} \%{ }^{236} \mathrm{U}$. Two plastic scintillation detectors (5.08-cm OD, 2.54-cm long) are located adjacent to the sphere and are positioned $180^{\circ}$ apart. The MCNP input file is given in Fig. 6.5 , and the extra data file contents for the frequency domain calculations are given in Fig. 6.6. The MCNP input file for this simulation is very similar to the one presented in Fig. 6.1 except the detectors have been changed from lithium glass detectors to plastic scintillation detectors whose neutron response is due to neutron scatter instead of neutron capture.

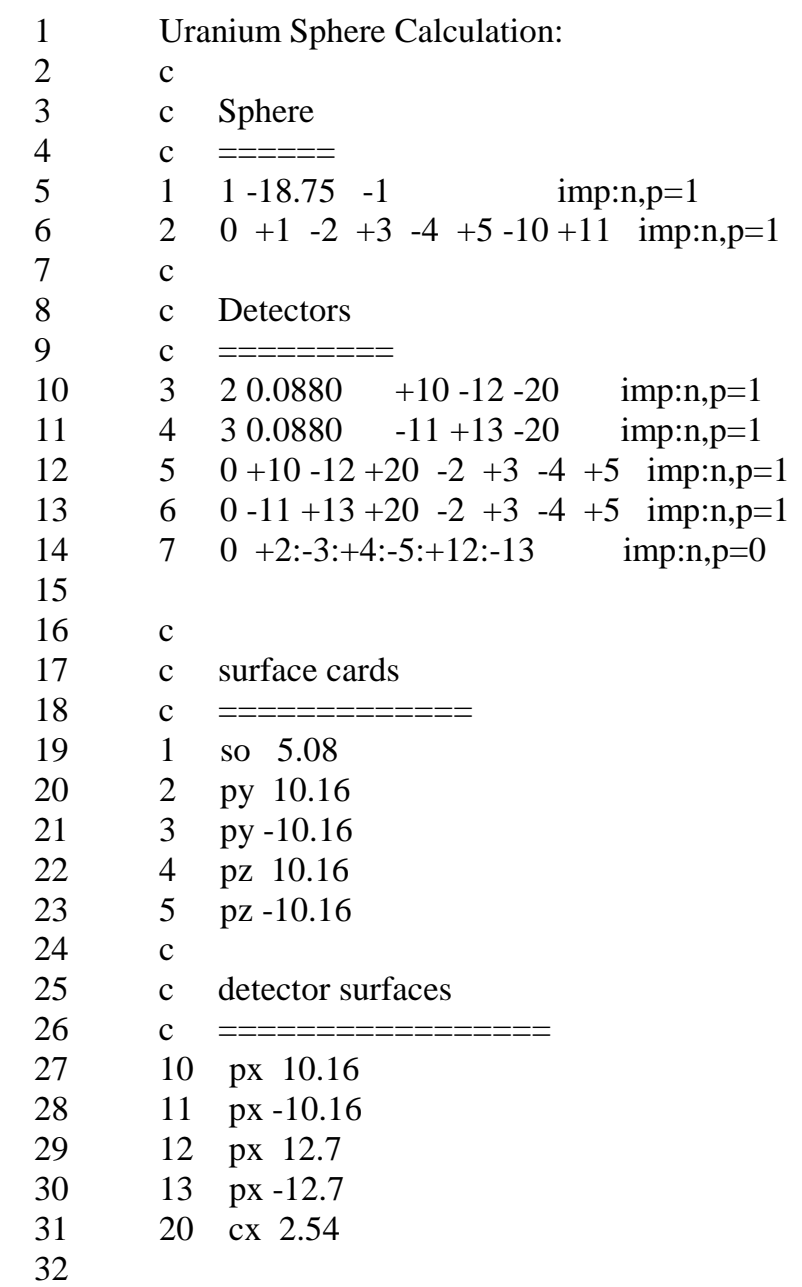

Figure 6.5. MCNP-DSP input file elalb.i. 


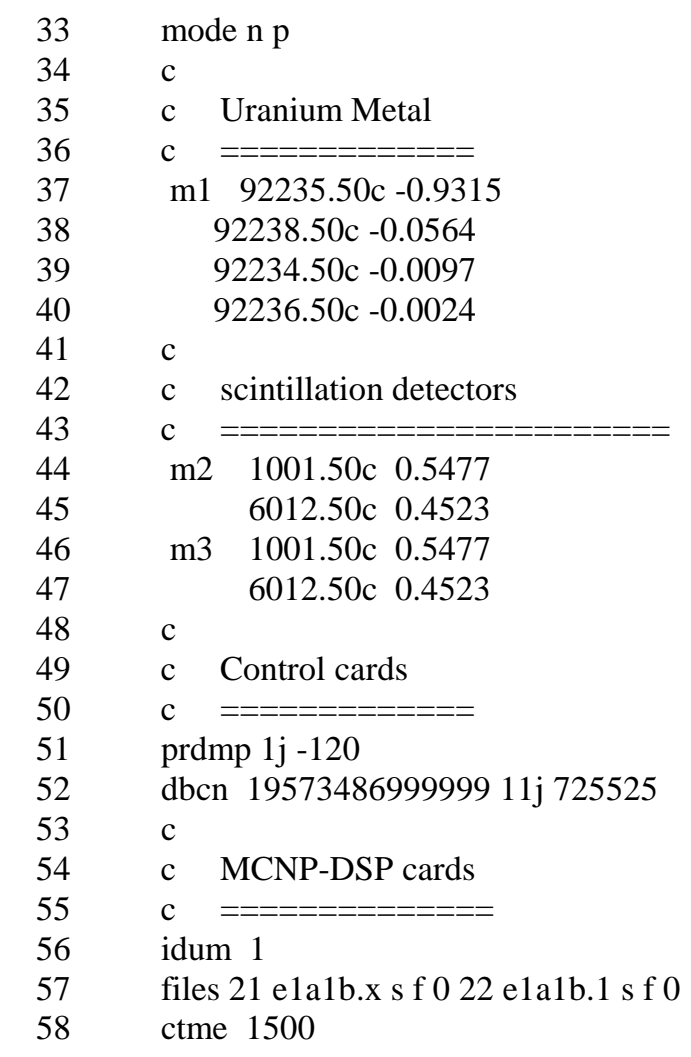

Figure 6.5. (continued).

\section{Description of Input File E1A1B.I}

Line 1: $\quad$ The first line of the input file is the title line.

Lines 2-14: These are the cell description cards. The cell cards are used to describe the composition of a cell and the surfaces that bound the particular cell. The cells must be numbered sequentially, and cells that are not used in the model must be removed. Otherwise, MCNP-DSP will produce errors in the calculations.

Lines 16-31: These lines contain the surface description cards. For this simple problem the surfaces consist of planes and cylinders. Note that the surface numbers do not have to be listed sequentially.

Lines 32-52: These are the data cards. Lines 32 through 47 describe the materials used in the calculations. The materials must be numbered sequentially. No materials can be skipped nor should unused materials be added to the input file. Lines 48 through 52 are the control cards for this problem. The prdmp card on line 51 is used to control how often data are written to the MCNP-DSP output files. The $d b c n$ card on line 52 is used to change the 
random number seed for the MCNP-DSP because the output data for several MCNP-DSP calculations are typically combined together.

Lines 53-58: These lines are used to execute the MCNP-DSP calculations. If the first entry on the idum card is 1, the MCNP-DSP calculation is executed and other tallies are not accumulated. The files card is used to specify two files. The first file (e1a1b.x) contains the extra data parameters and is opened as a sequential, formatted file on unit 21 . The second file (e1a1b.1) is the MCNP-DSP output file. The output file is opened as a sequential formatted file on unit 22. Both files must be entered as sequential and formatted files. The record length is zero for sequential files.

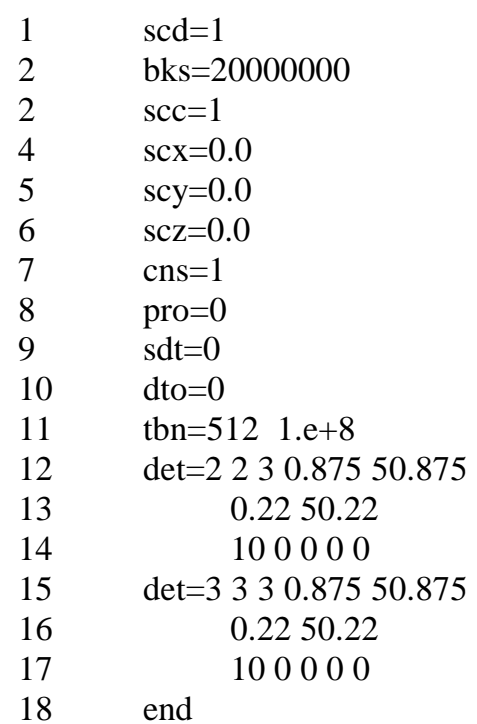

Figure 6.6. MCNP-DSP extra data file elalb.x.

\section{Description of Extra Data File E1A1B.X}

Line 1: $\quad$ One source disintegration per block $(s c d)$ in this example.

Line 2: $\quad$ The number of blocks to calculate $(b k s)$ is 20000000.

Lines 3-6: These lines represent the coordinates of the source. Line 3 is the source cell while lines 4-6 correspond to the origin of the source.

Line 7: $\quad$ This parameter specifies that the Madland-Nix prompt neutron spectrum is to be used in the simulation instead of the default Mannhart corrected Maxwellian spectrum. 
Line 8: This line specifies that this is a frequency analysis calculation.

Line 9: $\quad$ This specifies that the source dead time is zero nanoseconds.

Line 10: This line specifies that the dead time option be disabled.

Line 11: This line specifies the number of time channels as 512 with a sampling rate of $100 \mathrm{MHz}$.

Lines 12-17: These lines describe the detectors in the problem. The source is detector 1 and does not need to be labeled. This problem has two detectors, 2 and 3. Both detectors are scatter detectors. Detector 2 is material 2 and type 3 while detector 3 is material 3 and type 3 . The scatter detector requires both neutron and gamma ray thresholds. The lower and upper neutron thresholds are 0.875 and $50.875 \mathrm{MeV}$ respectively. The lower and upper gamma ray thresholds are 0.22 and $50.22 \mathrm{MeV}$ respectively. The pulse generation time is set to $10 \mathrm{~ns}$. There are no energy bins in this calculation and there are no neutron or gamma ray background counts for the detector. The dead time for each detector is zero. For the frequency domain calculations, at least two detectors ( 2 and 3 ) must be listed in the extra data file.

Line 16: The end parameter is used to specify the end of the extra file. This option could have also been omitted.

The output files for this example are not provided because they are very similar to those provided in section 6.1.1.1. This example was provided to demonstrate the specifications required for scatter detectors such as plastic scintillation detectors.

\subsubsection{Uranium Metal Sphere with Composite Detectors}

This example demonstrates a simulation with a ${ }^{252} \mathrm{Cf}$ source that is located within a highly enriched uranium metal sphere. Again, the uranium metal density is $18.75 \mathrm{~g} / \mathrm{cm}^{3}$, and the uranium isotopic content is $93.15 \mathrm{wt} \%{ }^{235} \mathrm{U}, 5.64 \mathrm{wt} \%{ }^{238} \mathrm{U}, 0.97 \mathrm{wt} \%{ }^{234} \mathrm{U}$, and $0.24 \mathrm{wt} \%{ }^{236} \mathrm{U}$. Two composite detectors are located adjacent to the sphere and are positioned $180^{\circ}$ apart. Each detector is comprised of a lithium glass (5.08-cm OD, 2.54-cm long) and plastic (5.08-cm OD, 2.54-cm long) scintillation detector. The MCNP input file is given in Fig. 6.7, and the extra data file contents for the frequency domain calculations are given in Fig. 6.8. The MCNP input file for this simulation is very similar to the one presented in Fig. 6.1 except the detectors have been changed from lithium glass detectors to composite detectors. Each component of the composite detector is specified in the MCNP-DSP extra data file. 
Uranium Sphere Calculation:

$1 \quad 1-18.75-1 \quad$ imp:n, $\mathrm{p}=1$

$20+1-2+3-4+5-10+11$ imp:n,p=1

$320.0813+10-12-20 \quad$ imp:n, $\mathrm{p}=1$

$430.0813 \quad-11+13-20 \quad$ imp:n, $p=1$

$540.0880+12-14-20 \quad$ imp:n,p=1

$650.0880 \quad-13+15-20 \quad$ imp:n, $\mathrm{p}=1$

$70+10-14+20-2+3-4+5$ imp:n,p=1

$80-11+15+20-2+3-4+5$ imp:n, $\mathrm{p}=1$

$90+2:-3:+4:-5:+14:-15 \quad$ imp:n, $p=0$

11

$12 \quad 1 \quad$ so 5.08

$13 \quad 2$ py 12.7

$14 \quad 3 \quad$ py -12.7

$\begin{array}{llll}15 & 4 & \mathrm{pz} & 12.7\end{array}$

$16 \quad 5 \quad \mathrm{pz}-12.7$

$17 \quad 10 \quad$ px 12.7

$18 \quad 11$ px -12.7

$19 \quad 12$ px 15.24

$20 \quad 13$ px -15.24

$21 \quad 14$ px 17.78

$22 \quad 15$ px -17.78

$23 \quad 20 \quad$ cx $\quad 2.54$

24

25

mode $\mathrm{n} \mathrm{p}$

m1 92235.50c -0.9315

92238.50c -0.0564

$92234.50 \mathrm{c}-0.0097$

$92236.50 \mathrm{c}-0.0024$

m2 $3006.50 \mathrm{c} \quad 0.215791$

3007.55 c 0.009885

$8016.50 \mathrm{c} \quad 0.529568$

$12000.50 \mathrm{c} \quad 0.001820$

$13027.50 \mathrm{c} 0.017433$

14000.50 c 0.223942

m3 3006.50c 0.215791

3007.55 c 0.009885

$8016.50 \mathrm{c} \quad 0.529568$

12000.50c 0.001820

$13027.50 \mathrm{c} 0.017433$

14000.50 c 0.223942

m4 $1001.50 \mathrm{c} \quad 0.5477$

$6000.50 \mathrm{c} \quad 0.4523$

m5 $1001.50 \mathrm{c} \quad 0.5477$

$6000.50 \mathrm{c} 0.4523$

prdmp $1 \mathrm{j}-120$

dbcn 19573486999999 11j 725525

$\mathrm{c}$

c MCNP-DSP cards

c ==ニ==========

idum 1

files 21 e1a1c.x s f 022 e1a1c. 1 s f 0

ctme 1500

Figure 6.7. MCNP-DSP input file ela1c.i. 


\section{Description of Input File E1A1C.I}

Line 1: The first line of the input file is the title line.

Lines 2-10: These are the cell description cards. The cell cards are used to describe the composition of a cell and the surfaces that bound the particular cell. The cells must be numbered sequentially, and cells that are not used in the model must be removed. Otherwise, MCNP-DSP will produce errors in the calculations.

Lines 12-23: These lines contain the surface description cards. For this simple problem the surfaces consist of planes and cylinders. Note that the surface numbers do not have to be listed sequentially.

Lines 25-47: These are the data cards. Line 25 indicates that this is a dual neutron and photon calculation. Lines 26 through 45 describe the materials used in the calculations. The materials must be numbered sequentially. No materials can be skipped nor should unused materials be added to the input file. A separate material must be entered for each detector because the detector tallies are determined by the material in which interactions occur. Each material for a composite detector must be specified in the MCNP-DSP input file. Lines 46 and 47 are the control cards for this problem. The prdmp card on line 46 is used to control how often data are written to the MCNP-DSP output files. The $d b c n$ card on line 47 is used to change the random number seed for the MCNP-DSP because the output data for several MCNP-DSP calculations are typically combined together.

Lines 51-53: These lines are used to execute the MCNP-DSP calculations. If the first entry on the idum card is 1, the MCNP-DSP calculation is executed and other tallies are not accumulated. The files card is used to specify two files. The first file (e1a1c.x) contains the extra data parameters and is opened as a sequential, formatted file on unit 21 . The second file (e1a1c.1) is the MCNP-DSP output file. The output file is opened as a sequential formatted file on unit 22. Both files must be entered as sequential and formatted files. The record length is zero for sequential files. 


\begin{tabular}{|c|c|c|}
\hline 1 & \multicolumn{2}{|l|}{$\mathrm{scd}=1$} \\
\hline 2 & \multicolumn{2}{|c|}{$\mathrm{bks}=20000000$} \\
\hline 3 & \multicolumn{2}{|c|}{$\mathrm{scc}=1$} \\
\hline 4 & \multicolumn{2}{|l|}{$\operatorname{sc} x=0.0$} \\
\hline 5 & \multicolumn{2}{|l|}{ scy $=0.0$} \\
\hline 6 & \multicolumn{2}{|l|}{$\mathrm{scz}=0.0$} \\
\hline 7 & \multicolumn{2}{|l|}{$\mathrm{cns}=1$} \\
\hline 8 & \multicolumn{2}{|l|}{ pro $=0$} \\
\hline 9 & \multicolumn{2}{|l|}{$\mathrm{sdt}=0$} \\
\hline 10 & \multicolumn{2}{|l|}{$\mathrm{dto}=0$} \\
\hline 11 & \\
\hline 12 & \multicolumn{2}{|c|}{$\operatorname{det}=222 \quad 0.22 \quad 50.22$} \\
\hline 13 & \multirow{2}{*}{\multicolumn{2}{|c|}{ 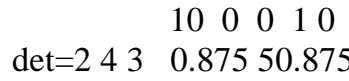 }} \\
\hline 14 & & \\
\hline 15 & \multicolumn{2}{|c|}{$\begin{array}{ll}0.22 & 50.22\end{array}$} \\
\hline 16 & \multirow{3}{*}{$\operatorname{det}=332$} & $\begin{array}{lllll}10 & 0 & 0 & 0 & 0\end{array}$ \\
\hline 17 & & $0.22 \quad 50.22$ \\
\hline 18 & & $\begin{array}{llllll}10 & 0 & 0 & 1 & 0\end{array}$ \\
\hline 19 & \multirow[t]{3}{*}{ det $=353$} & 0.87550 .875 \\
\hline 20 & & 0.2250 .22 \\
\hline 21 & & $\begin{array}{llllll}10 & 0 & 0 & 0 & 0\end{array}$ \\
\hline 22 & \multicolumn{2}{|l|}{ end } \\
\hline
\end{tabular}

Figure 6.8. MCNP-DSP extra data file elalc.x.

Description of Extra Data File E1A1C.X

Line 1: $\quad$ One source disintegration per block $(s c d)$ in this example.

Line 2: $\quad$ The number of blocks to calculate $(b k s)$ is 20000000 .

Lines 3-6: These lines represent the coordinates of the source. Line 3 is the source cell while lines 4-6 correspond to the origin of the source.

Line 7: $\quad$ This parameter specifies that the Madland-Nix prompt neutron spectrum is to be used in the simulation instead of the default Mannhart corrected Maxwellian spectrum.

Line 8: $\quad$ This line specifies that this is a frequency analysis calculation.

Line 9: $\quad$ This specifies that the source dead time is zero nanoseconds.

Line 10: This line specifies that the dead time option be disabled.

Line 11: This line specifies the number of time channels as 512 with a sampling rate of $100 \mathrm{MHz}$.

Lines 12-21: These lines describe the detectors in the problem. The source is detector 1 and does not need to be labeled. This problem has two detectors, 2 and 3. Both detectors are composite detectors. Detectors 2 and 3 consist of a 
lithium glass and the plastic scintillation detectors. Each component has the same detector identification number but different materials and detector types. One component of detector 2 is comprised of a lithium glass scintillation detector that is material 2 and is a capture detector (type 2 ). The other component of detector 2 is the plastic scintillation detector that is material 4 and is a scatter detector (type 3). The lithium glass detector response is affected by gamma rays; therefore, the gamma ray thresholds must be provided because this is a dual neutron and photon simulation. The lower and upper gamma ray thresholds are 0.22 and 50.22 $\mathrm{MeV}$ respectively. The pulse generation time is set to $10 \mathrm{~ns}$. There are no neutron or gamma ray background counts for the detector. The neutron capture efficiency is 1 for each detector, and the dead time for each detector is zero. The scatter detector requires both neutron and gamma ray thresholds. The lower and upper neutron thresholds are 0.875 and 50.875 $\mathrm{MeV}$ respectively. The lower and upper gamma ray thresholds are 0.22 and $50.22 \mathrm{MeV}$ respectively. The pulse generation time is set to $10 \mathrm{~ns}$. There are no energy bins in this calculation and there are no neutron or gamma ray background counts for the detector. The dead time for each detector is zero. For the frequency domain calculations, at least two detectors ( 2 and 3 ) must be listed in the extra data file.

Line 22: $\quad$ The end parameter is used to specify the end of the extra file. This option could have also been omitted.

\subsubsection{Uranyl Nitrate Solution with ${ }^{3} \mathrm{He}$ Detectors}

This example demonstrates the use of ${ }^{3} \mathrm{He}$ neutron detectors in the frequency analysis calculation of a hypothetical uranyl nitrate solution. The solution is contained within an acrylic cylinder with a $25.0825-\mathrm{cm}$ ID and a $53.34-\mathrm{cm}$ height. The solution is filled to a height of $25.4 \mathrm{~cm}$. The uranyl nitrate solution contains 0.29330 gram of uranium per gram of solution. The solution density is $1.64320 \mathrm{~g} / \mathrm{cm}^{3}$ and has a free acid content $<0.1 \mathrm{wt} \% \mathrm{HNO}_{3}$. The uranium isotopic content is $93.20 \mathrm{wt} \%{ }^{235} \mathrm{U}, 5.37 \mathrm{wt} \%$

${ }^{238} \mathrm{U}, 1.02 \mathrm{wt} \%{ }^{234} \mathrm{U}$, and $0.41 \mathrm{wt} \%{ }^{236} \mathrm{U}$. The source is specified as being in the center of the solution in the calculations; however, the geometry of the source is not included in the model. Two ${ }^{3} \mathrm{He}$ neutron detectors are adjacent to the radial surface of the cylinder and positioned $180^{\circ}$ apart. The MCNP input file is given in Fig. 6.9, and the extra data file contents for the frequency domain calculations are given in Fig. 6.10. 
c

c

c Uranyl Nitrate Solution

c =======================

$\begin{array}{lllllll}1 & 3 & 0.086239 & +1 & -2 & -32 & +33\end{array}$

$-70+71 \quad$ imp:n=1

$2 \begin{array}{llllll}2 & 1 & 0.0978989 & +2 & -3 & -54 \quad \text { imp:n=1 }\end{array}$

$\begin{array}{llllll}0 & 0 & +3 & -4 & -54 \quad \text { imp:n=1 }\end{array}$

$\mathrm{c}$

c Acrylic Tank

c $===========$

$4 \quad 20.106467 \quad+2 \quad-4+54 \quad-55 \quad$ imp: $\mathrm{n}=1$

$\begin{array}{lllllll}5 & 0 & +2 & -4 & +55 & -30+31\end{array}$

$-32+33 \quad$ imp:n=1

C

c Detectors

c $=========$

$6 \quad 0 \quad+15-16-40 \quad i m p: n=1$ \$ Lower Inactive

74 7.38444e-5 +16 -17 -40 imp:n=1 \$ Right Detector

$8 \quad 0 \quad+17-18-40 \quad$ imp:n=1 \$ Upper Inactive

$960.0603167 \quad(+2 \quad-15 \quad-41):$

$(+15-18+40-41):$

(+18 -19 -41) imp:n=1 \$ Al Around Detector

$100+15-16-42 \quad$ imp:n=1 \$ Lower Inactive

$1157.38444 \mathrm{e}-5+16-17-42 \quad$ imp:n=1 \$ Left Detector

$120+17-18-42 \quad$ imp:n=1 \$ Upper Inactive

$\begin{array}{llllll}13 & 6 & 0.0603167 & (+2 & -15 & -43):\end{array}$

$(+15-18+42-43):$

$(+18-19-43) \quad$ imp:n=1 \$ Al Around Detector

$140 \quad+2-19+41+30-70$

$-32+33 \quad$ imp:n=1 \$ Void Right Detector

$150 \quad+2-19+43-31+71$

$-32+33 \quad$ imp:n=1 \$ Void Left Detector

$\begin{array}{llllll}16 & 0 & +19 & -4 & +30 & -70\end{array}$

$-32+33 \quad$ imp:n=1 \$ Void Right

$\begin{array}{llllll}17 & 0 & +19 & -4 & -31 & +71\end{array}$

$-32+33 \quad$ imp:n=1 \$ Void Left

$18 \quad 0 \quad-1:+4:+70:-71:+32:-33 \quad$ imp:n=0

c

c Z-planes

c $=======$

$1 \mathrm{pz}-0.635$

2 pz 0.0

3 pz 25.40

4 pz 53.34

$\mathrm{c}$

$15 \mathrm{pz} \quad 0.1397$

$16 \mathrm{pz} 1.7653$

$17 \mathrm{pz} 37.465$

$18 \mathrm{pz} 39.2303$

19 pz 39.37

Figure 6.9. MCNP-DSP input file e1a1d.i. 


\begin{tabular}{|c|c|c|c|}
\hline 54 & $\mathrm{c}$ & & \\
\hline 55 & $\mathrm{c}$ & X,Y-planes & \\
\hline 56 & $\mathrm{c}$ & $=======$ & \\
\hline 57 & 30 & py 13.49376 & \\
\hline 58 & 31 & py -13.49376 & \\
\hline 59 & 32 & px 13.49376 & \\
\hline 60 & 33 & px -13.49376 & \\
\hline 61 & $\mathrm{c}$ & & \\
\hline 62 & $\mathrm{c}$ & Z-cylinders & \\
\hline 63 & $\mathrm{c}$ & $==========$ & \\
\hline 64 & 40 & $\begin{array}{ccc}c / z & 0.0 & 16.0338\end{array}$ & 2.4003 \\
\hline 65 & 41 & $\mathrm{c} / \mathrm{z} \quad 0.0 \quad 16.0338$ & 2.54 \\
\hline 66 & 42 & $c / z \quad 0.0 \quad-16.0338$ & 2.4003 \\
\hline 67 & 43 & $\mathrm{c} / \mathrm{z} \quad 0.0 \quad-16.0338$ & 2.54 \\
\hline 68 & $\mathrm{c}$ & & \\
\hline 69 & 54 & cz 12.54125 & \\
\hline 70 & 55 & cz 13.49375 & \\
\hline 71 & $\mathrm{c}$ & & \\
\hline 72 & $\mathrm{c}$ & Outer Boundaries & \\
\hline 73 & $\mathrm{c}$ & $=====$ & \\
\hline 74 & 70 & py 19.0 & \\
\hline 75 & 71 & py -19.0 & \\
\hline 76 & & & \\
\hline 77 & $\bmod$ & de $n$ & \\
\hline 78 & $\mathrm{c}$ & & \\
\hline 79 & $\mathrm{c}$ & Material Cards & \\
\hline 80 & c & $======$ = = = = = & \\
\hline 81 & $\mathrm{c}$ & & \\
\hline 82 & $\mathrm{c}$ & Uranyl Nitrate & \\
\hline 83 & $\mathrm{c}$ & $==============$ & \\
\hline 84 & $\mathrm{~m} 1$ & $\begin{array}{lll}1001.50 \mathrm{c} & 0.5738816\end{array}$ & \\
\hline 85 & & $8016.50 \mathrm{c} \quad 0.3880981$ & \\
\hline 86 & & $7014.50 \mathrm{c} \quad 0.0254020$ & \\
\hline 87 & & $92235.50 \mathrm{c} \quad 0.0117555$ & \\
\hline 88 & & 92238.50 c 0.0006688 & \\
\hline 89 & & 92234.50 c 0.0001292 & \\
\hline 90 & & $92236.50 \mathrm{c} \quad 0.0000515$ & \\
\hline 91 & & $26000.50 \mathrm{c} 0.0000100$ & \\
\hline 92 & & 24000.50 c 0.0000017 & \\
\hline 93 & & $13027.50 \mathrm{c} 0.0000007$ & \\
\hline 94 & & $28000.50 \mathrm{c} \quad 0.0000007$ & \\
\hline 95 & & $25055.50 \mathrm{c} 0.0000002$ & \\
\hline 96 & $\mathrm{mt} 1$ & lwtr.01t & \\
\hline 97 & c & & \\
\hline 98 & c & Acrylic Tank & \\
\hline 99 & $\mathrm{c}$ & $===========$ & \\
\hline 100 & $\mathrm{~m} 2$ & $\begin{array}{lll}1001.50 \mathrm{c} & 0.533333\end{array}$ & \\
\hline 101 & & $6000.50 \mathrm{c} \quad 0.333334$ & \\
\hline 102 & & $8016.50 \mathrm{c} \quad 0.133333$ & \\
\hline 103 & $\mathrm{mt} 2$ & poly.01t & \\
\hline
\end{tabular}

Figure 6.9. (continued). 


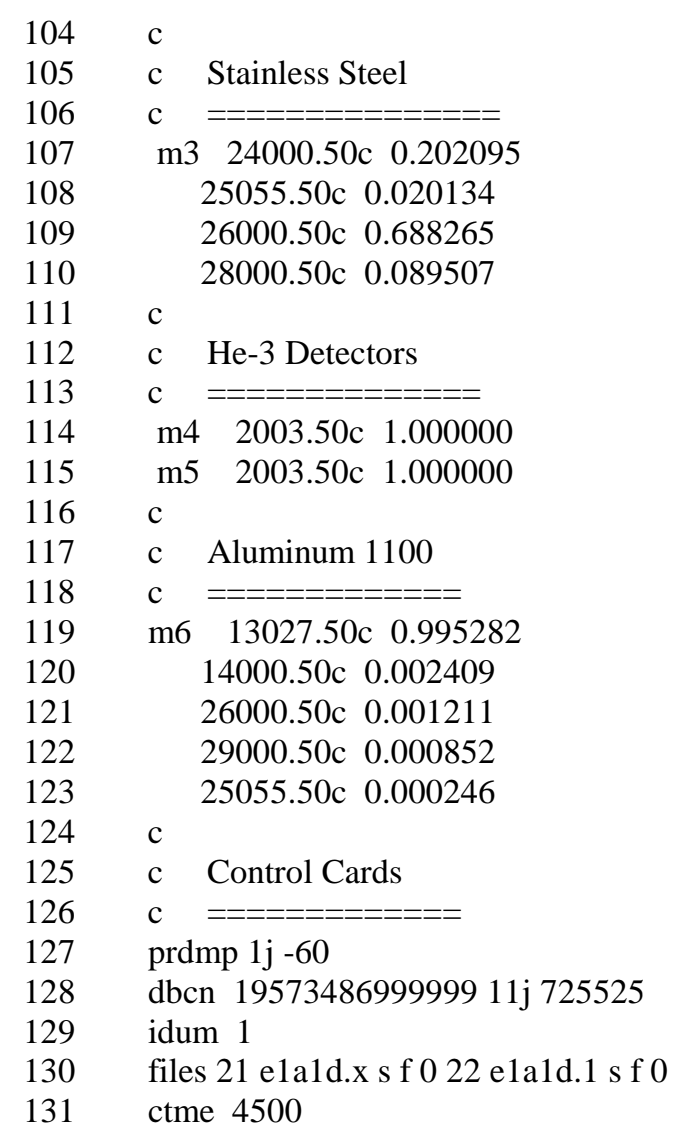

Figure 6.9. (continued).

\section{Description of Input File E1A1D.I}

Line 1: $\quad$ The first line of the input file is the title line.

Lines 2-39: These are the cell description cards. The cell cards are used to describe the composition of a cell and the surfaces that bound the particular cell. The cells must be numbered sequentially, and cells that are not used in the model must be removed. Otherwise, MCNP-DSP will produce errors in the calculations.

Lines 41-75: These lines contain the surface description cards. For this simple problem the surfaces consist of planes and cylinders. Note that the surface numbers do not have to be listed sequentially.

Lines 77-128: These are the data cards. Line 77 indicates that this is a neutron calculation. Lines 78 through 123 describe the materials used in the calculations. The materials must be numbered sequentially. No materials can be skipped nor should unused materials be added to the 
input file. A separate material must be entered for each detector because the detector tallies are determined by the material in which interactions occur. Lines 127 and 128 are the control cards for this problem. The prdmp card on line 127 is used to control how often data are written to the MCNP-DSP output files. The $d b c n$ card on line 128 is used to change the random number seed for the MCNP-DSP because the output data for several MCNP-DSP calculations are typically combined together.

Lines 130-131: $\quad$ These lines are used to execute the MCNP-DSP calculations. If the first entry on the idum card is 1, the MCNP-DSP calculation is executed and other tallies are not accumulated. The files card is used to specify two files. The first file (e1a1d.x) contains the extra data parameters and is opened as a sequential, formatted file on unit 21 . The second file (e1a1d.1) is the MCNP-DSP output file. The output file is opened as a sequential formatted file on unit 22. Both files must be entered as sequential and formatted files. The record length is zero for sequential files.

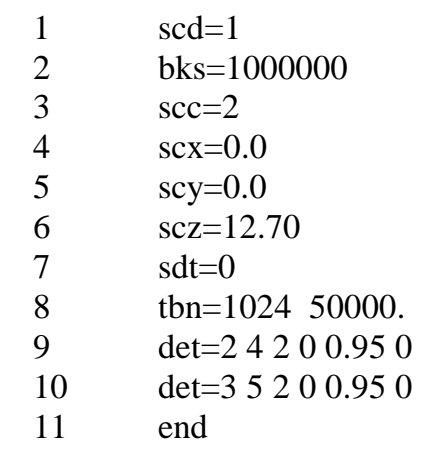

Figure 6.10. MCNP-DSP extra data file ela1d.x.

\section{Description of Extra Data File E1A1D.X}

Line 1: $\quad$ One source disintegration per block $(s c d)$ in this example.

Line 2: $\quad$ The number of blocks to calculate $(b k s)$ is 1000000.

Lines 3-6: These lines represent the coordinates of the source. Line 3 is the source cell while lines 4-6 correspond to the origin of the source.

Line 7: $\quad$ This specifies that the source dead time is zero nanoseconds.

Line 8: $\quad$ This line specifies the number of time channels as 1024 with a sampling rate of $50 \mathrm{kHz}$. 
Lines 9-10: These lines describe the detectors in the problem. The source is detector 1 and does not need to be labeled. This problem has two detectors, 2 and 3. Detector 2 is material 2 and is a capture detector (type 2). There are no neutron background counts for the detector. The neutron capture efficiency is 0.95 for each detector, and the dead time for each detector is zero.

Line 11: The end parameter is used to specify the end of the extra file. This option could have also been omitted.

\subsubsection{Uranium Metal Sphere with Plastic Scintillation Detectors and Continuous, Isotropic D-T Source}

This example demonstrates a simulation with a continuous, isotropic, D-T source that is located within a highly enriched uranium metal sphere. Again, the uranium metal density is $18.75 \mathrm{~g} / \mathrm{cm}^{3}$, and the uranium isotopic content is $93.15 \mathrm{wt} \%{ }^{235} \mathrm{U}, 5.64 \mathrm{wt} \%$ ${ }^{238} \mathrm{U}, 0.97 \mathrm{wt} \%{ }^{234} \mathrm{U}$, and $0.24 \mathrm{wt} \%{ }^{236} \mathrm{U}$. Two plastic scintillation detectors (5.08-cm OD, $2.54-\mathrm{cm}$ long) are located adjacent to the sphere and are positioned $180^{\circ}$ apart. The MCNP input file is given in Fig. 6.11, and the extra data file contents for the frequency domain calculations are given in Fig. 6.12. The MCNP input file for this simulation is the same as the one presented in Fig. 6.5 with the exception that the extra files for MCNP-DSP have been changed. If the continuous, isotropic D-T source is used, the variable 'scd' in the extra data file corresponds to the number of source neutrons per block.

\section{Description of Input File E1A2A.I}

The input file provided in Fig. 6.11 is essentially the same as the one provided in Fig. 6.5 with the exception of the different MCNP-DSP data files.

Lines 50-52: These lines are used to execute the MCNP-DSP calculations. If the first entry on the idum card is 1, the MCNP-DSP calculation is executed and other tallies are not accumulated. The files card is used to specify two files. The first file (e1a2a.x) contains the extra data parameters and is opened as a sequential, formatted file on unit 21. The second file (e1a2a.1) is the MCNP-DSP output file. The output file is opened as a sequential formatted file on unit 22 . Both files must be entered as sequential and formatted files. The record length is zero for sequential files. 
c

c Detectors

c $=======$

$320.0880+10-12-20 \quad$ imp:n,p=1

$430.0880 \quad-11+13-20 \quad$ imp:n,p=1

$50+10-12+20-2+3-4+5$ imp:n,p=1

$60-11+13+20-2+3-4+5$ imp:n,p=1

$70+2:-3:+4:-5:+12:-13 \quad$ imp:n, $p=0$

$\mathrm{c}$

c surface cards

c =============

1 so 5.08

2 py 10.16

3 py -10.16

4 pz 10.16

$5 \mathrm{pz}-10.16$

10 px 10.16

11 px -10.16

12 px 12.7

13 px -12.7

20 cx 2.54

mode $\mathrm{n} \mathrm{p}$

c

c Uranium Metal

c =============

m1 $92235.50 \mathrm{c}-0.9315$

$92238.50 \mathrm{c}-0.0564$

$92234.50 \mathrm{c}-0.0097$

$92236.50 \mathrm{c}-0.0024$

c

c scintillation detectors

c =======================

m2 $1001.50 \mathrm{c} \quad 0.5477$

6012.50c 0.4523

m3 1001.50c 0.5477

6012.50 c 0.4523

c

c Control cards

c ============

prdmp $1 \mathrm{j}-120$

dbcn 19573486999999 11j 725525

c

c MCNP-DSP cards

c ============

idum 1

files 21 e1a2a.x s f 022 e1a2a.1 s f 0

ctme 1500

Figure 6.11. MCNP-DSP input file e1a2a.i . 


$$
\begin{aligned}
& \mathrm{scd}=1 \\
& \text { bks }=20000000 \\
& \mathrm{scc}=1 \\
& \operatorname{scx}=0.0 \\
& \mathrm{scy}=0.0 \\
& \mathrm{scZ}=0.0 \\
& \text { pro }=0 \\
& \mathrm{sdt}=0 \\
& \mathrm{dto}=0 \\
& \mathrm{stp}=1 \quad 0 \quad 0 \\
& \text { tbn }=512 \quad 1 . e+8 \\
& \text { det=2 } 230.87550 .875 \\
& 0.2250 .22 \\
& 100000 \\
& \text { det=3 } 330.87550 .875 \\
& 0.2250 .22 \\
& 100000 \\
& \text { end }
\end{aligned}
$$

\section{Figure 6.12. MCNP-DSP extra input file e1a2a.x .}

\section{Description of Extra Data File E1A2A.X}

The only difference between Fig. 6.6 and Fig. 6.12 is that the option 'stp' has been specified instead of using the default ${ }^{252} \mathrm{Cf}$ source.

Line 1: $\quad$ One source disintegration per block $(s c d)$ in this example.

Line 2: $\quad$ The number of blocks to calculate $(b k s)$ is 20000000.

Lines 3-6: These lines represent the coordinates of the source. Line 3 is the source cell while lines 4-6 correspond to the origin of the source.

Line 7: This specifies that this is a frequency analysis calculation $($ pro $=0)$.

Line 8: $\quad$ This specifies that the source dead time is zero $(s d t=0)$.

Line 9: $\quad$ This option specifies that the dead time option is not invoked $(d t o=0)$.

Line 10: This line specifies that the source type is a D-T source that is operating in the continuous mode with an isotropic distribution of neutrons from the source.

Line 11: This line specifies the number of time channels as 512 with a sampling rate of $100 \mathrm{MHz}$.

Lines 12-17: These lines describe the detectors in the problem. The source is detector 1 and does not need to be labeled. This problem has two detectors, 2 and 3. 
Both detectors are scatter detectors. Detector 2 is material 2 and type 3 while detector 3 is material 3 and type 3 . The scatter detector requires both neutron and gamma ray thresholds. The lower and upper neutron thresholds are 0.875 and $50.875 \mathrm{MeV}$ respectively. The lower and upper gamma ray thresholds are 0.22 and $50.22 \mathrm{MeV}$ respectively. The pulse generation time is set to $10 \mathrm{~ns}$. There are no energy bins in this calculation and there are no neutron or gamma ray background counts for the detector. The dead time for each detector is zero. For the frequency domain calculations, at least two detectors ( 2 and 3 ) must be listed in the extra data file.

Line 16: The end parameter is used to specify the end of the extra file. This option could have also been omitted.

6.1.1.6 Uranium Metal Spheres with Plastic Scintillation Detectors and Pulsed, AngularDependent D-T Source

The extra data file provided in Fig. 6.12 can be modified to simulate a pulsed, angular-dependent D-T source. Simply simply changing line 10 can change the D-T source from a continuous, isotropic source to a pulsed, angular-dependent source. The modified extra data file is provided in Fig. 6.13. If the pulsed, angular-dependent D-T source is used, the variable ' $s c d$ ' in the extra data file corresponds to the number of source neutrons per pulse.

\begin{tabular}{|c|c|}
\hline 1 & $\operatorname{scd}=15$ \\
\hline 2 & $\mathrm{bks}=20000000$ \\
\hline 3 & $\operatorname{scx}=0.0$ \\
\hline 4 & $\mathrm{scy}=0.0$ \\
\hline 5 & $s c z=-7.62$ \\
\hline 6 & pro $=0$ \\
\hline 7 & $\mathrm{sdt}=0$ \\
\hline 8 & $\mathrm{dto}=0$ \\
\hline 9 & $\mathrm{stp}=1 \quad 1 \quad 5000010000$ \\
\hline 10 & 100015 \\
\hline 11 & $\mathrm{tbn}=512 \quad 1 . e+7$ \\
\hline 12 & det=2 230.87550 .875 \\
\hline 13 & 0.2250 .22 \\
\hline 14 & 100000 \\
\hline 15 & det=3 330.87550 .875 \\
\hline 16 & 0.2250 .22 \\
\hline 17 & 100000 \\
\hline 18 & end \\
\hline
\end{tabular}

Figure 6.13. MCNP-DSP extra input file $e 1 a 2 b . x$. 


\section{Description of Extra Data File E1A2B.X}

The only difference between Fig. 6.13 and Fig. 6.12 is that the option 'stp' has been modified and the number of source disintegrations has been changed.

Line 1: $\quad$ Fifteen source disintegration per pulse $(s c d)$ in this example.

Line 2: $\quad$ The number of blocks to calculate $(b k s)$ is 20000000.

Lines 3-5: These lines represent the coordinates of the source. Lines 3-5 correspond to the origin of the source. Note that the source position has been changed to correspond to a point below the sphere. From this point the source neutrons will be directed with the angular distribution provided on the 'stp' card.

Line 6: $\quad$ This specifies that this is a frequency analysis calculation $(p r o=0)$.

Line 7: $\quad$ This specifies that the source dead time is zero $(s d t=0)$.

Line 8: $\quad$ The dead time is not invoked $(d t o=0)$.

Lines 9-10: These lines specify that the source type is a D-T source. The first entry (1) specifies that the source is a D-T source while the second entry (1) specifies that the source is a pulsed source. The third entry is the source repetition rate in $\mathrm{Hz}(50000)$ and the fourth entry is the pulse width in nanoseconds (10000). The source specification is continued on line 10. The first entry (1) on line 10 is used to specify that the source is a conical source. The next three entries are the reference vector for the source. The entries 001 are the direction cosines for the reference vector. Therefore, the source is directed up the positive z-axis. The next entry (5) is the halfangle for emission of neutrons from the source origin. This neutron source has a conical angular distribution that varies $\pm 5^{\circ}$ from the reference vector that is directed up the positive $\mathrm{z}$-axis.

Line 11: $\quad$ This line specifies the number of time channels as 512 with a sampling rate of $100 \mathrm{MHz}$.

Lines 12-17: These lines describe the detectors in the problem and are the same as those provided in Fig. 6.13. 


\subsubsection{Delta Phase Plutonium Metal Sphere with at ${ }^{252} \mathrm{Cf}$ Source}

This example demonstrates the use of a simulation with a plutonium metal sphere with a ${ }^{252} \mathrm{Cf}$ source. The delta phase plutonium sphere has a density of $15.48 \mathrm{~g} / \mathrm{cm}^{3}$ and is comprised of $90 \mathrm{wt} \%{ }^{239} \mathrm{Pu}, 9 \mathrm{wt} \%{ }^{240} \mathrm{Pu}$, and $1 \mathrm{wt} \%{ }^{241} \mathrm{Pu}$. The outer radius of the plutonium sphere is $7.5 \mathrm{~cm}$. Two plastic scintillation detectors are located $7.5-\mathrm{cm}$ away from the surface of the sphere and positioned $180^{\circ}$ apart. The ${ }^{252} \mathrm{Cf}$ source is specified as being located in the center of the sphere although the source is not physically modeled in the geometry. This simulation assumes that the ${ }^{252} \mathrm{Cf}$ source is a $1 \mu \mathrm{g}$ source $\left(6.14 \cdot 10^{5}\right.$ spontaneous fissions per second per $\mu \mathrm{g}$ ); therefore, the number of source disintegrations per block is $1.257\left(6.14 \cdot 10^{5} * 1024 / 5 \cdot 10^{8}\right)$. The ${ }^{240} \mathrm{Pu}$ decays via spontaneous fission with a decay constant of $1.63918 \cdot 10^{-19}$. The mass of ${ }^{240} \mathrm{Pu}$ in the sphere is $2462 \mathrm{~g}$. The spontaneous fission rate is simply $1.012 \cdot 10^{6}$ fissions per second $\left(1.63918 \cdot 10^{-19} *\right.$ $2462.0 * 6.02214 \cdot 10^{23} / 240.05$ ). Therefore, approximately 2.073 spontaneous fissions occur in each block. The MCNP input file is provided in Fig. 6.15 and the extra data file is provided in Fig. 6.16.

\section{Description of Input File E1A3.I}

Line 1: $\quad$ The first line of the input file is the title line.

Lines 2-13: These are the cell description cards. The cells must be numbered sequentially, and cells that are not used in the model must be removed. Otherwise, MCNP-DSP will produce errors in the calculations.

Lines 15-31: These lines contain the surface description cards.

Lines 33-45: These are the data cards. The materials must be numbered sequentially. No materials can be skipped nor should unused materials be added to the input file. A separate material must be entered for each detector because the detector tallies are determined by the material in which interactions occur. The $\operatorname{prdmp}$ card is used to control how often data are written to the MCNP-DSP output files. The $d b c n$ card is used to change the random number seed for the MCNP-DSP because the output data for several MCNP-DSP calculations are typically combined together.

Lines 46-48: These lines are used to execute the MCNP-DSP calculations. If the first entry on the idum card is 1, the MCNP-DSP calculation is executed and other tallies are not accumulated. The files card is used to specify two files. The first file (e1a3.x) contains the extra data parameters and is opened as a sequential, formatted file on unit 21. The second file (e1a3.1) is the MCNP-DSP output file. The output file is opened as a sequential formatted file on unit 22. Both files must be entered as sequential and formatted files. The record length is zero for sequential files. 
Plutonium Sphere: Outer Radius $7.5 \mathrm{~cm}$

c Plutonium Shell

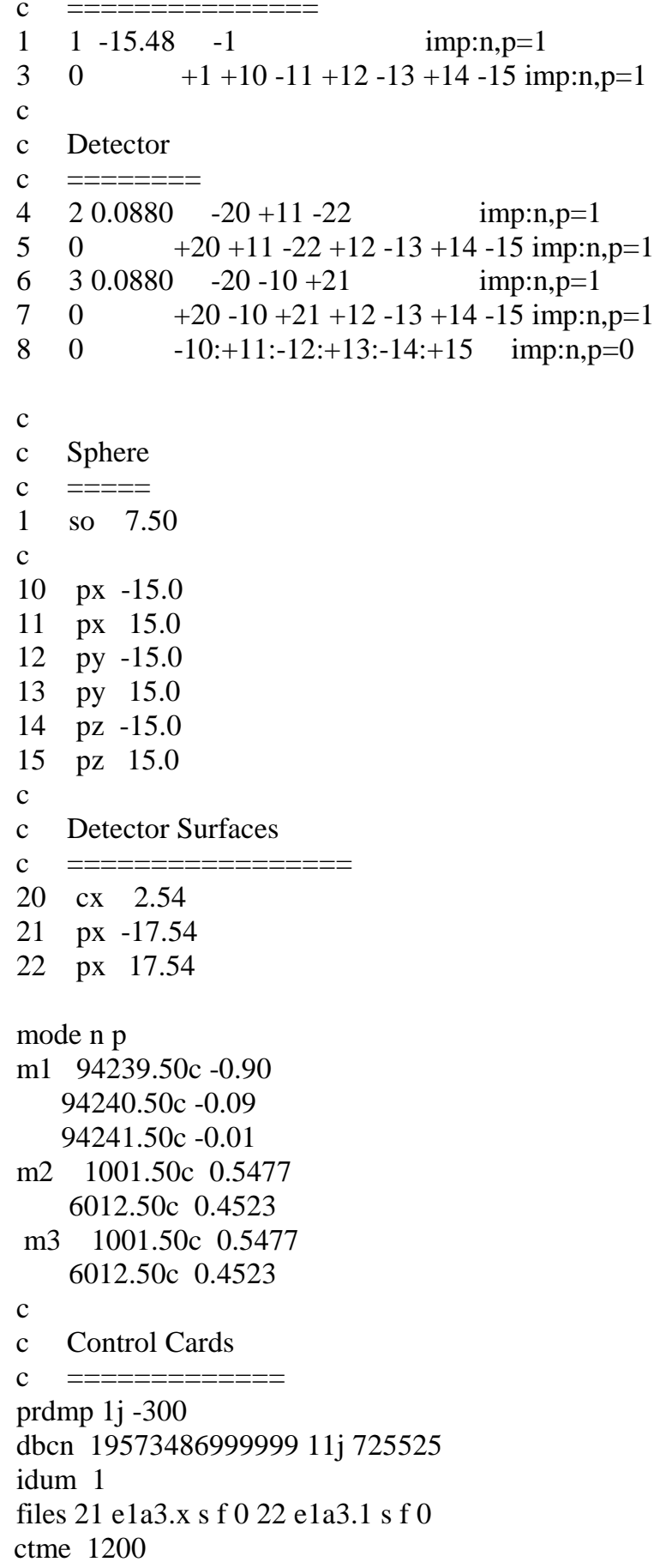

Figure 6.14. MCNP-DSP input file e1a3.i. 


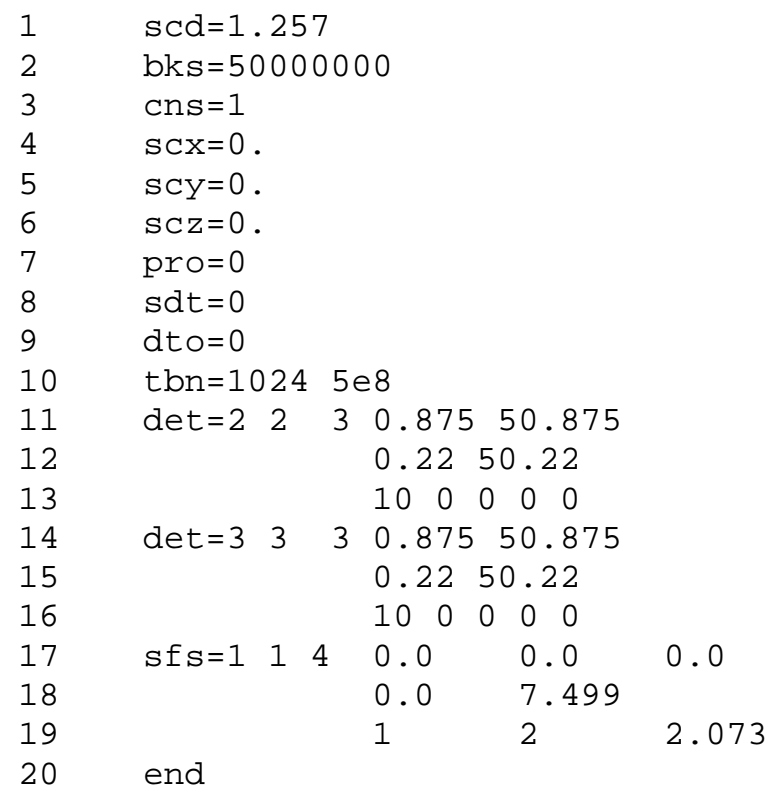

Figure 6.15. MCNP-DSP extra input file e1a3.x.

\section{Description of Extra Data File E1A3.X}

Line 1: $\quad$ The source disintegrations per block $(s c d)$ is 1.257 in this example.

Line 2: $\quad$ The number of blocks to calculate $(b k s)$ is 50000000.

Line 3: $\quad$ This parameter (cns) specifies the use of the Madland-Nix prompt neutron energy spectrum for ${ }^{252} \mathrm{Cf}$.

Lines 4-6: These lines represent the coordinates of the ${ }^{252} \mathrm{Cf}$ source.

Line 7: $\quad$ This specifies that this is a frequency analysis calculation $($ pro $=0)$.

Line 8: $\quad$ This specifies that the source dead time is zero $(s d t=0)$.

Line 9: $\quad$ The dead time is not invoked $(d t o=0)$.

Line 10: $\quad$ This line specifies the number of time channels as 1024 with a sampling rate of $500 \mathrm{MHz}$.

Lines 11-16: These lines describe the detectors in the problem. The source is detector 1 and does not need to be labeled. This problem has two detectors, 2 and 3 . Both detectors are scatter detectors. Detector 2 is material 2 and type 3 while detector 3 is material 3 and type 3 . The scatter detector requires both neutron and gamma ray thresholds. The lower and upper neutron 
thresholds are 0.875 and $50.875 \mathrm{MeV}$ respectively. The lower and upper gamma ray thresholds are 0.22 and $50.22 \mathrm{MeV}$ respectively. The pulse generation time is set to $10 \mathrm{~ns}$. There are no energy bins in this calculation and there are no neutron or gamma ray background counts for the detector. The dead time for each detector is zero. For the frequency domain calculations, at least two detectors (2 and 3) must be listed in the extra data file.

Lines 17-19: These lines are used to specify the inherent spontaneous fission source for ${ }^{240} \mathrm{Pu}$. The first parameter is the total number of spontaneous fission sources. In this example, only one inherent spontaneous fission source is selected. The next entry is the number of volumes that contain inherent spontaneous fission sources. In this example, only one volume contains the inherent source. The next entry is the source volume type that is simply a spherical source. The origin of the inherent source volume is provided next. The source parameters are continued on line 18. The first entry is the inner radius of the sphere (type 4 volume) and the next entry is the outer radius of the sphere. Note that the outer dimensions cannot correspond exactly to one of the surfaces of the problem because the source is a volume source and not a surface source. The source parameters are continued on line 19. The first entry on line 19 is the number of inherent spontaneous fission sources in this volume and is equal to one. The next entry is the spontaneous fission type (type $2={ }^{240} \mathrm{Pu}$ ). The next entry is the number of spontaneous fission per block. Note that the exact number of ${ }^{252} \mathrm{Cf}$ spontaneous fissions per block and the exact number of inherent spontaneous fissions per block did not have to be entered. Arbitrary numbers could have been used; however, the ratio of ${ }^{252} \mathrm{Cf}$ to inherent $\left({ }^{252} \mathrm{Cf} /{ }^{240} \mathrm{Pu}\right)$ spontaneous fissions must be maintained.

\subsubsection{Passive Simulations}

\subsubsection{Nested Plutonium Shells with Plastic Scintillation Detectors}

This example demonstrates the use of a passive frequency analysis simulation with a multi-volume, inherent spontaneous fission source. The geometry consists of a 5.0-cm OD delta phase plutonium sphere surrounded by a delta phase plutonium shell that has 9.0-cm ID and a 15.0-cm OD. The delta phase plutonium sphere has a density of $15.48 \mathrm{~g} / \mathrm{cm}^{3}$ and is comprised of $90 \mathrm{wt} \%{ }^{239} \mathrm{Pu}, 9 \mathrm{wt} \%{ }^{240} \mathrm{Pu}$, and $1 \mathrm{wt} \%{ }^{241} \mathrm{Pu}$. Two plastic scintillation detectors are located $7.5-\mathrm{cm}$ away from the surface of the outer shell and positioned $180^{\circ}$ apart. The only source for this problem is the inherent plutonium source. The MCNP input file for this problem is provided in Fig. 6.16 and the MCNPDSP extra data file is provided in Fig. 6.17. 


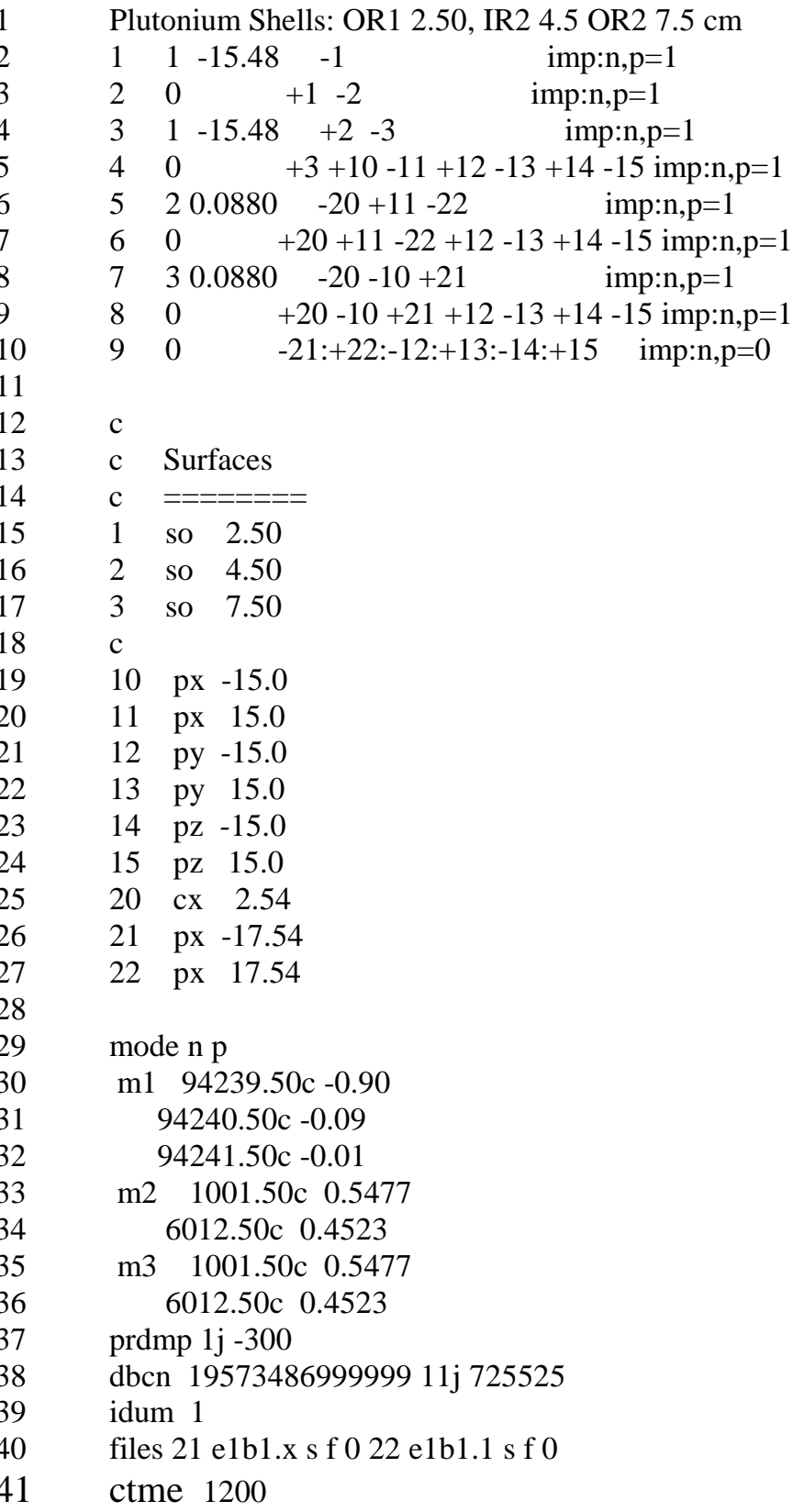

Figure 6.16. MCNP-DSP input file $e$ 1b1.i.

\section{Description of Input File E1B1.I}

Line 1: The first line of the input file is the title line.

Lines 2-10: These are the cell description cards. The cells must be numbered sequentially, and cells that are not used in the model must be removed. Otherwise, MCNP-DSP will produce errors in the calculations. 
Lines 12-27: These lines contain the surface description cards.

Lines 29-38: These are the data cards. The materials must be numbered sequentially. No materials can be skipped nor should unused materials be added to the input file. A separate material must be entered for each detector because the detector tallies are determined by the material in which interactions occur. The prdmp card is used to control how often data are written to the MCNP-DSP output files. The $d b c n$ card is used to change the random number seed for the MCNP-DSP because the output data for several MCNP-DSP calculations are typically combined together.

Lines 39-40: These lines are used to execute the MCNP-DSP calculations. If the first entry on the idum card is 1, the MCNP-DSP calculation is executed and other tallies are not accumulated. The files card is used to specify two files. The first file (e1b1.x) contains the extra data parameters and is opened as a sequential, formatted file on unit 21 . The second file (e1b1.1) is the MCNP-DSP output file. The output file is opened as a sequential formatted file on unit 22 .

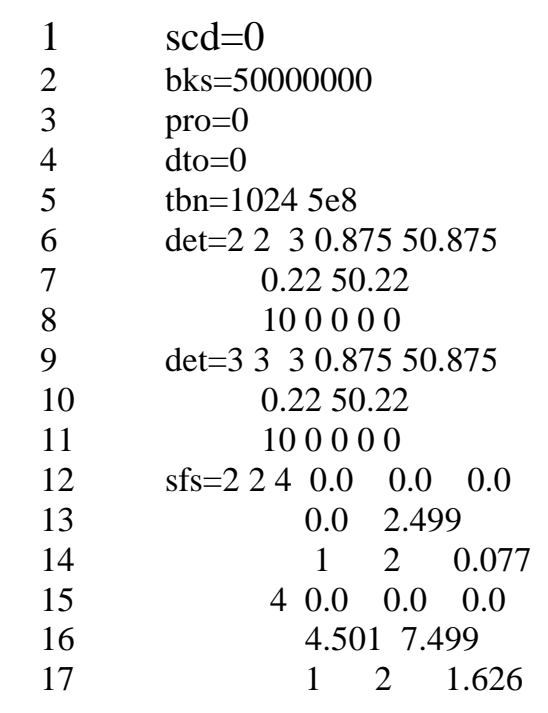

Figure 6.17. MCNP-DSP extra input file e1b1.x.

\section{Description of Extra Data File E1B1.X}

Line 1: $\quad$ The source disintegrations per block $(s c d)$ are zero in this example.

Line 2: $\quad$ The number of blocks to calculate $(b k s)$ is 5000000 . 
Line 3: This specifies that this is a frequency analysis calculation $($ pro $=0)$.

Line 4: $\quad$ The dead time is not invoked $(d t o=0)$.

Line 5: This line specifies the number of time channels as 1024 with a sampling rate of $500 \mathrm{MHz}$.

Lines 6-11: These lines describe the detectors in the problem. The source is detector 1 and does not need to be labeled. This problem has two detectors, 2 and 3. Both detectors are scatter detectors. Detector 2 is material 2 and type 3 while detector 3 is material 3 and type 3 . The scatter detector requires both neutron and gamma ray thresholds. The lower and upper neutron thresholds are 0.875 and $50.875 \mathrm{MeV}$ respectively. The lower and upper gamma ray thresholds are 0.22 and $50.22 \mathrm{MeV}$ respectively. The pulse generation time is set to $10 \mathrm{~ns}$. There are no energy bins in this calculation and there are no neutron or gamma ray background counts for the detector. The dead time for each detector is zero.

Lines 12-17: These lines are used to specify the inherent spontaneous fission source for ${ }^{240} \mathrm{Pu}$. The first parameter is the total number of spontaneous fission sources. In this example, two inherent spontaneous fission sources are selected. The next entry is the number of volumes that contain inherent spontaneous fission sources. In this example, two volumes contain the inherent source. The next entry is the source volume type that is simply a spherical source. The origin of the inherent source volume is provided next. The source parameters are continued on line 13. The first entry is the inner radius of the sphere (type 4 volume) and the next entry is the outer radius of the sphere. Note that the outer dimensions cannot correspond exactly to one of the surfaces of the problem because the source is a volume source and not a surface source. The source parameters are continued on line 14 . The first entry on line 14 is the number of inherent spontaneous fission sources in this volume and is equal to one. The next entry is the spontaneous fission type (type $2={ }^{240} \mathrm{Pu}$ ). The next entry is the number of spontaneous fission per block. On line 15, the source parameters for the second volume are specified. The first entry is the volume type (type 4 volume) and is followed by the origins of the second volume. The next entries on line 16 are the inner and outer radii of the second volume. Finally, the number of inherent spontaneous fission sources for the second volume is specified along with the type and number of spontaneous fission events per block. Note that although arbitrary numbers could have been entered, the actual numbers of spontaneous fission events per block per volume have been entered. It is important to remember that the ratio of spontaneous fission events per block must be maintained. 


\subsubsection{Adjacent Plutonium Cylinders with Plastic Scintillation Detectors}

This example demonstrates the use of a passive frequency analysis simulation with a multi-volume, inherent spontaneous fission source. The geometry consists of two identical $10-\mathrm{cm}$ tall, $20-\mathrm{cm}$ OD, delta phase plutonium cylinders spaced $30 \mathrm{~cm}$ apart. The delta phase plutonium sphere has a density of $15.48 \mathrm{~g} / \mathrm{cm}^{3}$ and is comprised of 90 wt $\%{ }^{239} \mathrm{Pu}, 9 \mathrm{wt} \%{ }^{240} \mathrm{Pu}$, and $1 \mathrm{wt} \%{ }^{241} \mathrm{Pu}$. Two plastic scintillation detectors are positioned near the cylinders. The only source for this problem is the inherent plutonium source. The MCNP input file for this problem is provided in Fig. 6.186 and the MCNPDSP extra data file is provided in Fig. 6.19.

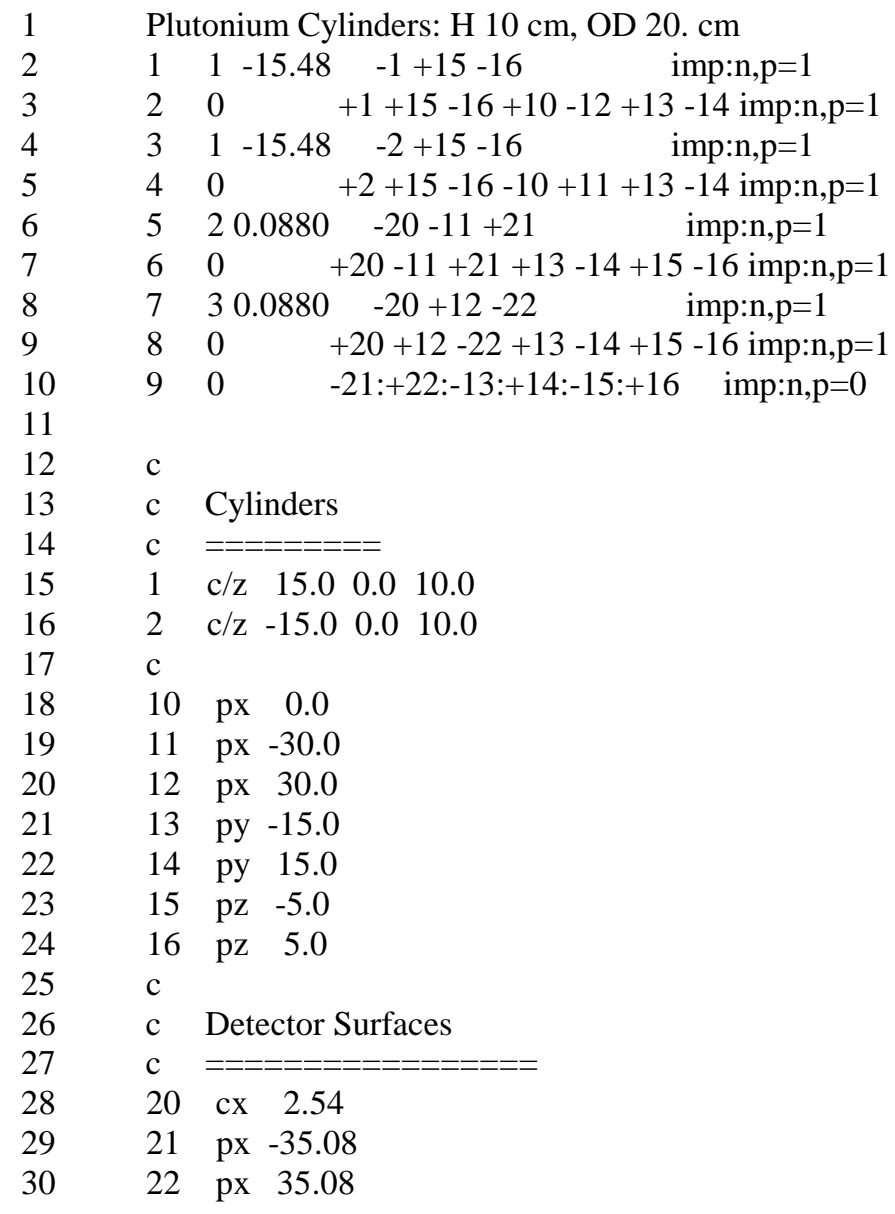

Figure 6.18. MCNP-DSP input file $e$ 1b2.i. 


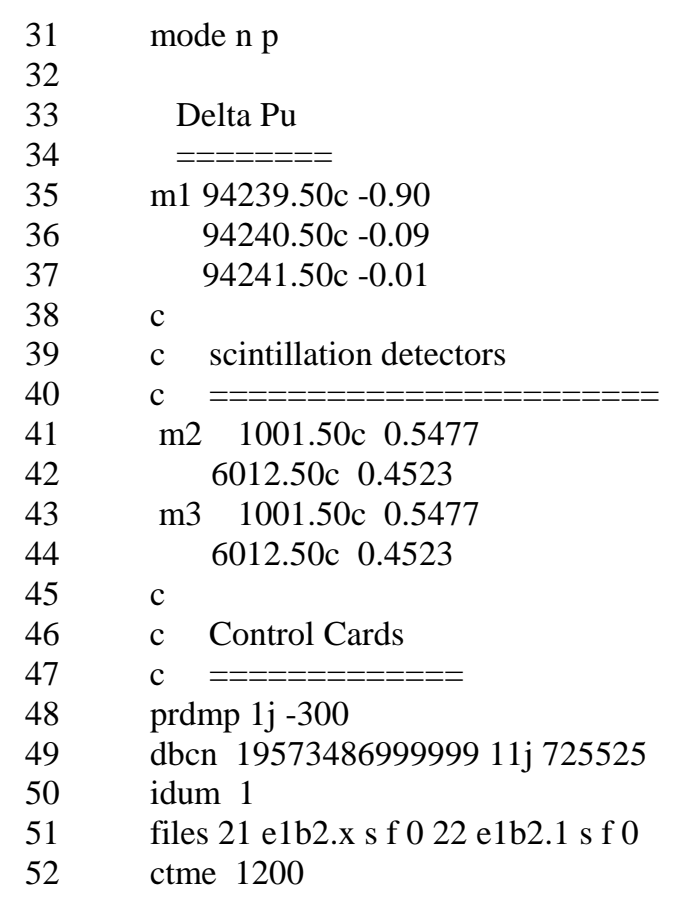

Figure 6.18. (continued).

\section{Description of Input File E1B2.I}

Line 1: $\quad$ The first line of the input file is the title line.

Lines 2-10: These are the cell description cards. The cells must be numbered sequentially, and cells that are not used in the model must be removed. Otherwise, MCNP-DSP will produce errors in the calculations.

Lines 12-30: These lines contain the surface description cards.

Lines 31-49: These are the data cards. The materials must be numbered sequentially. No materials can be skipped nor should unused materials be added to the input file. A separate material must be entered for each detector because the detector tallies are determined by the material in which interactions occur. The $\operatorname{prdmp}$ card is used to control how often data are written to the MCNP-DSP output files. The $d b c n$ card is used to change the random number seed for the MCNP-DSP because the output data for several MCNP-DSP calculations are typically combined together. 
Lines 50-51: These lines are used to execute the MCNP-DSP calculations. If the first entry on the idum card is 1, the MCNP-DSP calculation is executed and other tallies are not accumulated. The files card is used to specify two files. The first file (e1b2.x) contains the extra data parameters and is opened as a sequential, formatted file on unit 21. The second file (e1b2.1) is the MCNP-DSP output file. The output file is opened as a sequential formatted file on unit 22 .

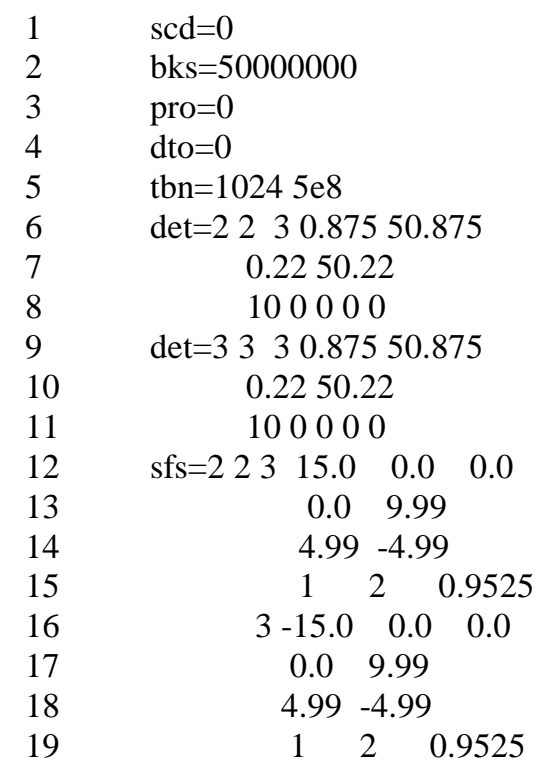

Figure 6.19. MCNP-DSP extra input file e1b2.x. Description of Extra Data File E1B2.X

Line 1: $\quad$ The source disintegrations per block $(s c d)$ are zero in this example.

Line 2: $\quad$ The number of blocks to calculate $(b k s)$ is 50000000 .

Line 3: $\quad$ This specifies that this is a frequency analysis calculation $($ pro $=0)$.

Line 4: $\quad$ The dead time is not invoked $(d t o=0)$.

Line 5: This line specifies the number of time channels as 1024 with a sampling rate of $500 \mathrm{MHz}$.

Lines 6-11: These lines describe the detectors in the problem. The source is detector 1 and does not need to be labeled. This problem has two detectors, 2 and 3. Both detectors are scatter detectors. Detector 2 is material 2 and type 3 while detector 3 is material 3 and type 3 . The scatter detector requires both 
neutron and gamma ray thresholds. The lower and upper neutron thresholds are 0.875 and $50.875 \mathrm{MeV}$ respectively. The lower and upper gamma ray thresholds are 0.22 and $50.22 \mathrm{MeV}$ respectively. The pulse generation time is set to $10 \mathrm{~ns}$. There are no energy bins in this calculation and there are no neutron or gamma ray background counts for the detector. The dead time for each detector is zero.

Lines 12-19: These lines are used to specify the inherent spontaneous fission source for ${ }^{240} \mathrm{Pu}$. The first parameter is the total number of spontaneous fission sources. In this example, two inherent spontaneous fission sources are selected. The next entry is the number of volumes that contain inherent spontaneous fission sources. In this example, two volumes contain the inherent source. The next entry is the source volume type that is simply a cylindrical source parallel to the z-axis. The origin of the inherent source volume is provided next. The source parameters are continued on line 13. The first entry is the inner radius of the cylinder (type 3 volume) and the next entry is the outer radius of the cylinder. Note that the outer dimensions cannot correspond exactly to one of the surfaces of the problem because the source is a volume source and not a surface source. The source parameters are continued on line 14. The first entry is the upper z-axis plane and the second entry is the lower z-axis plane. The first entry on line 15 is the number of inherent spontaneous fission sources in this volume and is equal to one. The next entry is the spontaneous fission type (type $2={ }^{240} \mathrm{Pu}$ ). The next entry is the number of spontaneous fission per block. On line 16, the source parameters for the second volume are specified. The first entry is the volume type (type 3 volume) and is followed by the origins of the second volume. The next entries on line 17 are the inner and outer radii of the second volume. On line 18, the upper and lower z-axis planes are specified. Finally, the number of inherent spontaneous fission sources for the second volume is specified along with the type and number of spontaneous fission events per block on line 19. Note that although arbitrary numbers could have been entered, the actual numbers of spontaneous fission events per block per volume have been entered. It is important to remember that the ratio of spontaneous fission events per block must be maintained. 


\subsection{CORRELATION ANALYSIS SIMULATION}

This example demonstrates a simulation with a ${ }^{252} \mathrm{Cf}$ source that is located within a highly enriched uranium metal sphere. Again, the uranium metal density is $18.75 \mathrm{~g} / \mathrm{cm}^{3}$, and the uranium isotopic content is $93.15 \mathrm{wt} \%{ }^{235} \mathrm{U}, 5.64 \mathrm{wt} \%{ }^{238} \mathrm{U}, 0.97 \mathrm{wt} \%{ }^{234} \mathrm{U}$, and $0.24 \mathrm{wt} \%{ }^{236} \mathrm{U}$. Two plastic scintillation detectors $(5.08-\mathrm{cm} \mathrm{OD}, 2.54-\mathrm{cm}$ long) are located adjacent to the sphere and are positioned $180^{\circ}$ apart. The MCNP input file is given in Fig. 6.20 , and the extra data file contents for the frequency domain calculations are given in Fig. 6.21.

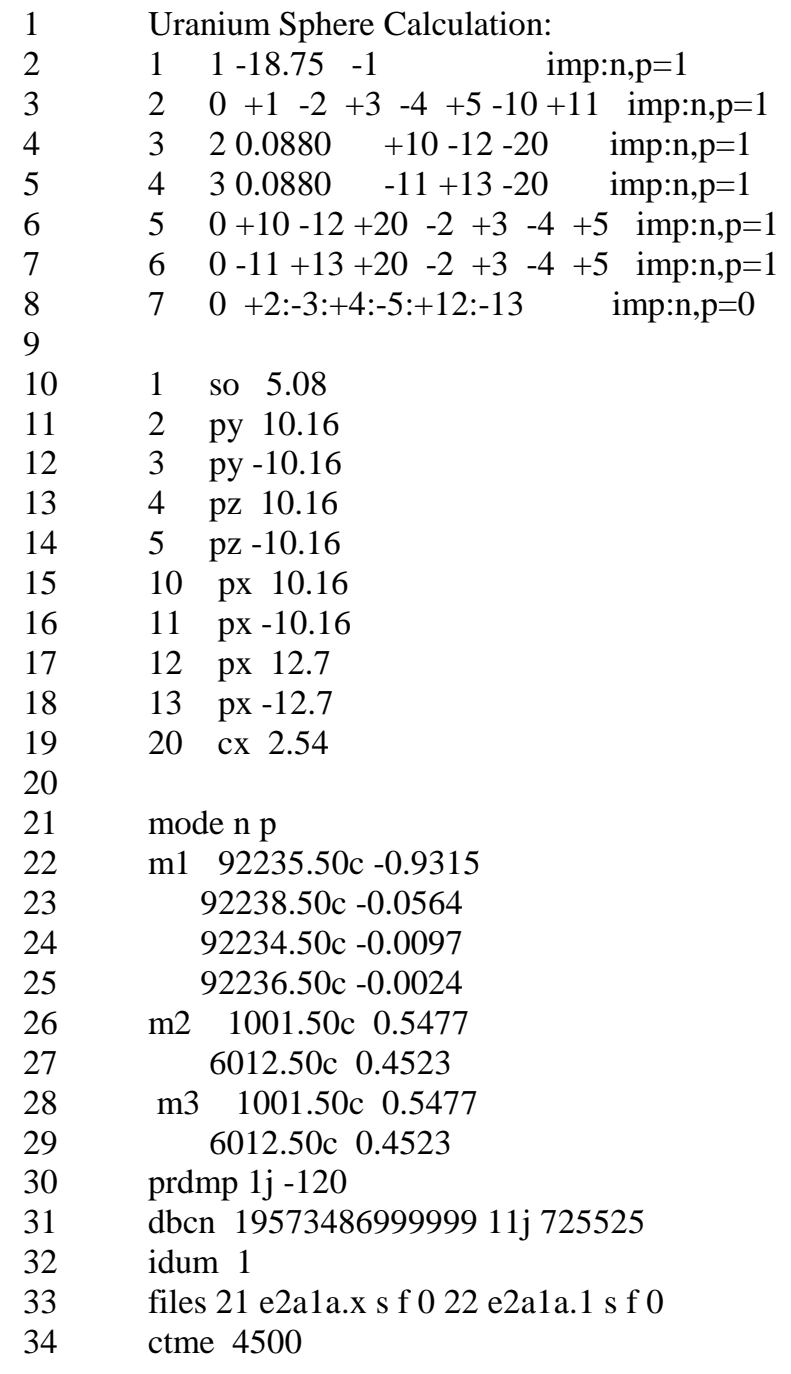

Figure 6.20. MCNP-DSP input file e2ala.i.

\section{Description of Input File E2A1A.I}

Line 1: $\quad$ The first line of the input file is the title line. 
Lines 2-8: These are the cell description cards. The cells must be numbered sequentially, and cells that are not used in the model must be removed. Otherwise, MCNP-DSP will produce errors in the calculations.

Lines 10-19: These lines contain the surface description cards.

Lines 21-31: These are the data cards. The materials must be numbered sequentially. No materials can be skipped nor should unused materials be added to the input file. The prdmp card is used to control how often data are written to the MCNP-DSP output files. The $d b c n$ card is used to change the random number seed for the MCNP-DSP because the output data for several MCNP-DSP calculations are typically combined together.

Lines 32-33: These lines are used to execute the MCNP-DSP calculations. If the first entry on the idum card is 1, the MCNP-DSP calculation is executed and other tallies are not accumulated. The files card is used to specify two files. The first file (e2a1a.x) contains the extra data parameters and is opened as a sequential, formatted file on unit 21 . The second file (e2a1a.1) is the MCNP-DSP output file. The output file is opened as a sequential formatted file on unit 22. Both files must be entered as sequential and formatted files. The record length is zero for sequential files.

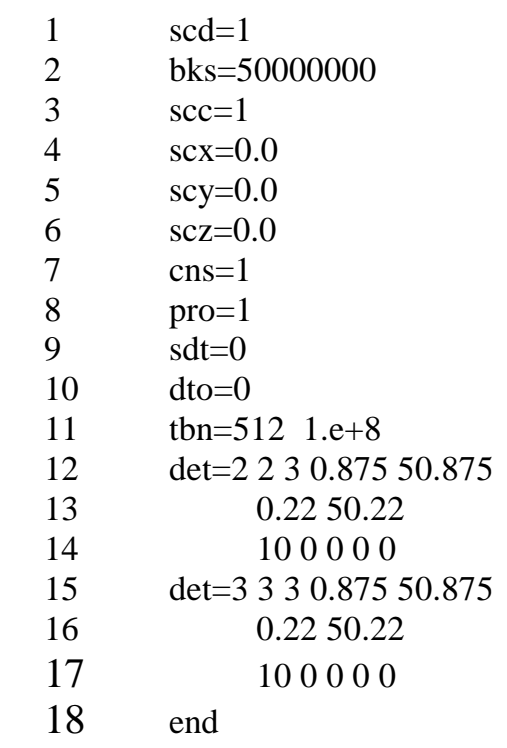

Figure 6.21. MCNP-DSP extra data file e2a1a.x.

\section{Description of Extra Data File E2A1A.X}

Line 1: $\quad$ One source disintegration per block $(s c d)$ in this example. 
Line 2: $\quad$ The number of blocks to calculate $(b k s)$ is 50000000 .

Lines 3-6: These lines represent the coordinates of the source. Line 3 is the source cell while lines 4-6 correspond to the origin of the source.

Line 7: $\quad$ This parameter specifies that the Madland-Nix prompt neutron spectrum is to be used in the simulation instead of the default Mannhart-corrected Maxwellian spectrum.

Line 8: This line specifies that this is a correlation analysis calculation.

Line 9: $\quad$ This specifies that the source dead time is zero nanoseconds.

Line 10: This line specifies that the dead time option be disabled.

Line 11: This line specifies the number of time channels as 512 with a sampling rate of $100 \mathrm{MHz}$.

Lines 12-15: These lines describe the detectors in the problem. The source is detector 1 and does not need to be labeled. This problem has two detectors, 2 and 3. Both detectors are scatter detectors. Detector 2 is material 2 and type 3 while detector 3 is material 3 and type 3 . The scatter detector requires both neutron and gamma ray thresholds. The lower and upper neutron thresholds are 0.875 and $50.875 \mathrm{MeV}$ respectively. The lower and upper gamma ray thresholds are 0.22 and $50.22 \mathrm{MeV}$ respectively. The pulse generation time is set to $10 \mathrm{~ns}$. There are no energy bins in this calculation and there are no neutron or gamma ray background counts for the detector. The dead time for each detector is zero. For the correlation analysis calculations, at least two detectors ( 2 and 3 ) must be listed in the extra data file.

Line 16: The end parameter is used to specify the end of the extra file. This option could have also been omitted.

Two or more output files are created when performing correlation analysis simulations. The first contains general information on the number of counts for each detector and is specified as the second data file in the MCNP input file. The other output file(s) contains the correlation signatures for the detectors. If the problem is a combined neutron-photon calculation as in the example, there will be three files created that contain the auto and cross correlation signatures for the detectors for the neutron response, the gamma response, and the combined neutron and gamma ray response. The general output filename as specified in Fig. 6.20 is e2ala.1. Three additional files will contain the auto and cross spectra. For a computer with the Unix operating system, these are e2ala.1.123.cor.n, e2ala.1.123.cor.p, and e2ala.1.123.cor.np, where the first file contains the spectra for the neutron response, the second file contains the spectra for the gamma response, and the third file contains the spectra for the combined neutron and 
gamma ray response as indicated by the last extension. The other filename extensions have distinct meanings. The first part that has the ' 123 ' indicates that this file contains the correlation signatures for the auto correlation of detectors \#1, \#2, and \#3, and the cross correlation functions between 1 and 2, 1 and 3, and 2 and 3. The 'cor' indicates that the file contains data that are correlation functions. Additional information concerning the output file names is provided in Appendix D. The correlation functions for multiple runs can be combined by combining the auto and cross correlation functions based on the number of blocks of data accumulated. If the user combines the correlation functions from several runs, the Monte Carlo calculation for each run must begin with a different random number seed that can be specified using the $d b c n$ card in the MCNP input file.

The MCNP-DSP summary output file (e2ala.1) for the correlation analysis simulation is similar to the summary output file for the frequency analysis simulation. A detailed description of the summary output file is provided in Section 6.1.1.1.

\section{Description of Output Correlation File E2A1A.1.123.COR.N}

Part of the MCNP-DSP output file e2ala.1.123.cor.n is provided in Fig. 6.22. This output file contains the time dependent auto and cross correlation functions for the neutron detections. The output file contains the frequency interval, the Nyquist frequency, the number of frequency points, and the number of data blocks that were accumulated during the calculation. The other entries in this file is as follows: auto correlation function of the source $(A C 11)$, auto correlation functions of the detectors ( $A C 22$ and $A C 33$ ), the cross correlation function between the source and detector 2 (CCor 12), the cross correlation between the source and detector 3 (CCor13), and the cross correlation between detectors (CCor 23). The correlation functions have been normalized by dividing the values at each time lag by $(N-|\tau|)$ with $N$ being the number of time points and $\tau$ being the lag index. Although this normalization produces an unbiased estimator of the correlation functions, the normalization is typically removed when computing covariance functions. The covariance function $\left(\operatorname{Cov}_{i j}(\tau)\right)$ can be computed as

$$
\operatorname{Cov}_{i j}(\tau)=\operatorname{Cor}_{i j}(\tau)(N-|\tau|)-C_{i} C_{j} / N / N_{B}
$$

In this expression $\operatorname{Cor}_{i j}(\tau)$ is the correlation function from output file, $C_{i}$ is the total number of counts for signal $i, C_{j}$ is the total number of counts for signal $j, N$ is the number of time bins in the simulation, and $N_{B}$ is the number of data blocks acquired. The user must normalize the data appropriately when comparing these simulations to other simulations or measurements. 


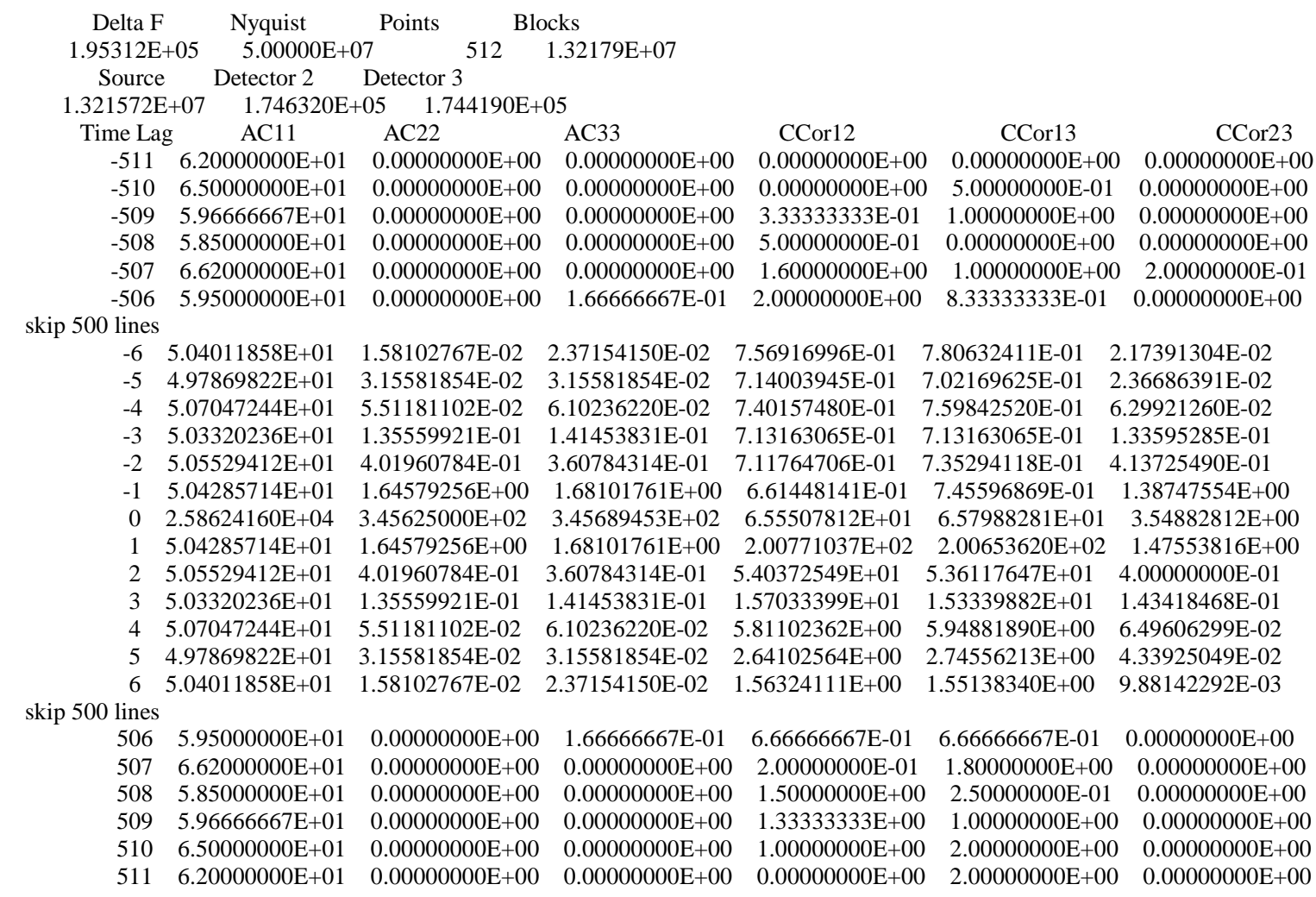

Figure 6.22. MCNP-DSP output file e2a1a.1.123.cor.n. 


\subsection{PULSED SOURCE SIMULATION}

The following example demonstrates the use of the pulsed source simulation with a ${ }^{252} \mathrm{Cf}$ source that is located within a highly enriched uranium metal sphere. Again, the uranium metal density is $18.75 \mathrm{~g} / \mathrm{cm}^{3}$, and the uranium isotopic content is $93.15 \mathrm{wt} \%$ ${ }^{235} \mathrm{U}, 5.64 \mathrm{wt} \%{ }^{238} \mathrm{U}, 0.97 \mathrm{wt} \%{ }^{234} \mathrm{U}$, and $0.24 \mathrm{wt} \%{ }^{236} \mathrm{U}$. Two plastic scintillation detectors (5.08-cm OD, 2.54-cm long) are located adjacent to the sphere and are positioned $180^{\circ}$ apart. The MCNP input file is given in Fig. 6.23, and the extra data file contents for the frequency domain calculations are given in Fig. 6.24. When performing pulsed source calculations, 'scd' is treated as the total number of ${ }^{252} \mathrm{Cf}$ source fissions, and ' $b k s$ ' must be equal to one. Because of the manner in which the pulsed neutron measurement executes, all data will be lost if it is interrupted as can be done with standard MCNP. Additionally, the dead time option cannot be used with the pulsed source simulation because of the manner in which the simulation is performed. The user should perform a correlation analysis simulation if the dead time option is desirable. The user is warned to select a reasonable value for 'scd' and to combine the detector responses for several runs. If several runs are combined, the user must start each run with a different random number seed using the $d b c n$ card in the MCNP input file.

\section{Description of Input File E3A1A.I}

Line 1: $\quad$ The first line of the input file is the title line.

Lines 2-8: $\quad$ These are the cell description cards. The cells must be numbered sequentially, and cells that are not used in the model must be removed. Otherwise, MCNP-DSP will produce errors in the calculations.

Lines 10-19: These lines contain the surface description cards.

Lines 21-31: These are the data cards. The materials must be numbered sequentially. No materials can be skipped nor should unused materials be added to the input file. The $p r d m p$ card is used to control how often data are written to the MCNP-DSP output files. The $d b c n$ card is used to change the random number seed for the MCNP-DSP because the output data for several MCNP-DSP calculations are typically combined together.

Lines 32-33: These lines are used to execute the MCNP-DSP calculations. If the first entry on the idum card is 1, the MCNP-DSP calculation is executed and other tallies are not accumulated. The files card is used to specify two files. The first file (e3a1a.x) contains the extra data parameters and is opened as a sequential, formatted file on unit 21 . The second file (e3a1a.1) is the MCNP-DSP output file. The output file is opened as a sequential formatted file on unit 22. Both files must be entered as sequential and formatted files. The record length is zero for sequential files. 
Uranium Sphere Calculation:

$1 \quad 1-18.75-1$

$20+1-2+3-4+5-10+11$

$320.0880+10-12-20$

$4 \quad 30.0880-11+13-20$

$5 \quad 0+10-12+20-2+3-4+5$

$60-11+13+20-2+3-4+5$

$70+2:-3:+4:-5:+12:-13$

1 so 5.08

2 py 10.16

3 py -10.16

$4 \mathrm{pz} 10.16$

$5 \mathrm{pz}-10.16$

10 px 10.16

11 px -10.16

12 px 12.7

13 px -12.7

$20 \quad$ cx 2.54

mode $\mathrm{n} p$

m1 92235.50c -0.9315

$92238.50 \mathrm{c}-0.0564$

$92234.50 \mathrm{c}-0.0097$

$92236.50 \mathrm{c}-0.0024$

$\mathrm{m} 2 \quad 1001.50 \mathrm{c} 0.5477$

6012.50c 0.4523

m3 $1001.50 \mathrm{c} 0.5477$

6012.50c 0.4523

prdmp $1 \mathrm{j}-120$

dbcn 19573486999999 11j 725525

idum 1

files 21 e3a1a.x s f 022 e3a1a.1 s f 0

ctme 3600 imp:n, $\mathrm{p}=1$

imp:n,p=1

imp:n,p=1

imp:n,p=1

imp: $n, p=1$

imp:n, $p=1$

imp:n, $\mathrm{p}=0$

Figure 6.23. MCNP-DSP input file e3ala.i. 


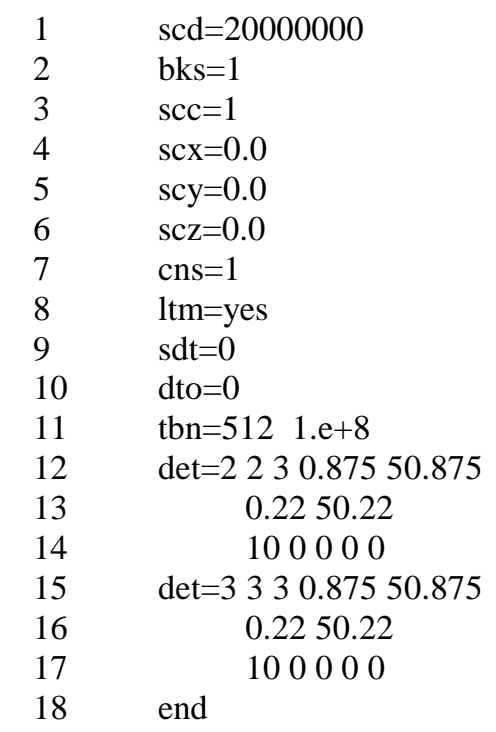

\section{Figure 6.24. MCNP-DSP extra data file e3a1a.x.}

\section{Description of Extra Data File 3A1A.X}

Line 1: $\quad$ The source disintegrations per block $(s c d)$ are 20000000 in this example.

Line 2: $\quad$ The number of blocks to calculate $(b k s)$ is 1.

Lines 3-6: These lines represent the coordinates of the source. Line 3 is the source cell while lines 4-6 correspond to the origin of the source.

Line 7: $\quad$ This parameter specifies that the Madland-Nix prompt neutron spectrum is to be used in the simulation instead of the default Mannhart-corrected Maxwellian spectrum.

Line 8: $\quad$ This line specifies that this is a pulsed source simulation.

Line 9: $\quad$ This specifies that the source dead time is zero nanoseconds.

Line 10: $\quad$ This line specifies that the dead time option be disabled.

Line 11: This line specifies the number of time channels as 512 with a sampling rate of $100 \mathrm{MHz}$. The time bin width is 10 nanoseconds.

Lines 12-15: These lines describe the detectors in the problem. The source is detector 1 and does not need to be labeled. This problem has two detectors, 2 and 3. Both detectors are scatter detectors. Detector 2 is material 2 and type 3 while detector 3 is material 3 and type 3 . The scatter detector requires both neutron and gamma ray thresholds. The lower and upper neutron thresholds are 0.875 and $50.875 \mathrm{MeV}$ respectively. The lower and upper gamma ray thresholds are 0.22 and $50.22 \mathrm{MeV}$ respectively. The pulse 
generation time is set to $10 \mathrm{~ns}$. There are no energy bins in this calculation and there are no neutron or gamma ray background counts for the detector. The dead time for each detector is zero. For the correlation analysis calculations, at least two detectors ( 2 and 3 ) must be listed in the extra data file.

Line 16: The end parameter is used to specify the end of the extra file. This option could have also been omitted.

Two output files are created when performing a pulsed source simulation. The first contains general information and is referred to as the MCNP-DSP summary output file. For this example, the output summary file is e3ala.1 as indicated on line 33 of Fig. 6.23. The second file contains the detector counts in each time interval after the source event. For a computer using the Unix operating system, the pulsed source time domain file would have the name e3ala.1.tim. The extension 'tim' is used to denote that this is time domain data.

\section{Description of Output Pulsed Source File E3A1A.1.TIM}

The output of the pulsed source time domain file is provided in Fig. 6.25. The output file contains the neutron, photon, and combined neutron and photon response for each detector. The output file has two columns that are separated by headers. The first line of the pulsed source output file contains the number of source events. The second line is a header line that describes the detector response. The first column contains the time of the midpoint of each time bin in nanoseconds. For this example the sampling rate was $100 \mathrm{MHz}$ that corresponds to a time bin width of $10 \mathrm{~ns}$. The second column contains the number of counts in each time interval. The count information is provided for the neutron, photon, and combined neutron and photon response for each detector because this is a dual neutron and photon calculation. 


\begin{tabular}{|c|c|}
\hline \multirow{2}{*}{\multicolumn{2}{|c|}{$\begin{array}{l}\text { Number of Source Events: } 19995984 \\
\text { Neutron Time Response for Detector \# } 2\end{array}$}} \\
\hline & \\
\hline $5.000000000000000 \mathrm{E}+00$ & $1.544740000000000 \mathrm{E}+05$ \\
\hline $1.500000000000000 \mathrm{E}+01$ & $8.031100000000000 \mathrm{E}+04$ \\
\hline $2.500000000000000 \mathrm{E}+01$ & $1.964000000000000 \mathrm{E}+04$ \\
\hline $3.500000000000000 \mathrm{E}+01$ & $6.450000000000000 \mathrm{E}+03$ \\
\hline $4.500000000000000 \mathrm{E}+01$ & $2.404000000000000 \mathrm{E}+03$ \\
\hline $5.500000000000000 \mathrm{E}+01$ & $9.530000000000000 \mathrm{E}+02$ \\
\hline $6.500000000000000 \mathrm{E}+01$ & $4.460000000000000 \mathrm{E}+02$ \\
\hline $7.500000000000000 \mathrm{E}+01$ & $2.130000000000000 \mathrm{E}+02$ \\
\hline $8.500000000000000 \mathrm{E}+01$ & $1.020000000000000 \mathrm{E}+02$ \\
\hline $9.500000000000000 \mathrm{E}+01$ & $5.000000000000000 \mathrm{E}+01$ \\
\hline $1.050000000000000 \mathrm{E}+02$ & $4.100000000000000 \mathrm{E}+01$ \\
\hline \multicolumn{2}{|c|}{ skip 501 lines } \\
\hline \multicolumn{2}{|c|}{ Photon Time Response for Detector \# 2} \\
\hline $5.000000000000000 \mathrm{E}+00$ & $1.895900000000000 \mathrm{E}+04$ \\
\hline $1.500000000000000 \mathrm{E}+01$ & $6.113000000000000 \mathrm{E}+03$ \\
\hline $2.500000000000000 \mathrm{E}+01$ & $1.856000000000000 \mathrm{E}+03$ \\
\hline $3.500000000000000 \mathrm{E}+01$ & 6.6000000000 \\
\hline $4.500000000000000 \mathrm{E}+01$ & $2.920000000000000 \mathrm{E}+02$ \\
\hline $5.500000000000000 \mathrm{E}+01$ & $1.250000000000000 \mathrm{E}+02$ \\
\hline $6.500000000000000 \mathrm{E}+01$ & $4.400000000000000 \mathrm{E}+01$ \\
\hline $7.500000000000000 \mathrm{E}+01$ & $3.300000000000000 \mathrm{E}+01$ \\
\hline $8.500000000000000 \mathrm{E}+01$ & $1.600000000000000 \mathrm{E}+01$ \\
\hline $9.500000000000000 \mathrm{E}+01$ & $2.000000000000000 \mathrm{E}+00$ \\
\hline $1.050000000000000 \mathrm{E}+02$ & $3.000000000000000 \mathrm{E}+00$ \\
\hline \multicolumn{2}{|c|}{ skip 501 lines } \\
\hline \multicolumn{2}{|c|}{ Neutron and Photon Time Response for Detector \# 2} \\
\hline $5.000000000000000 \mathrm{E}+00$ & $1.734330000000000 \mathrm{E}+05$ \\
\hline $1.500000000000000 \mathrm{E}+01$ & $8.642400000000000 \mathrm{E}+04$ \\
\hline $2.500000000000000 \mathrm{E}+01$ & $2.149600000000000 \mathrm{E}+04$ \\
\hline $3.500000000000000 \mathrm{E}+01$ & $7.110000000000000 \mathrm{E}+03$ \\
\hline $4.500000000000000 \mathrm{E}+01$ & $2.696000000000000 \mathrm{E}+03$ \\
\hline $5.500000000000000 \mathrm{E}+01$ & 1.078000000 \\
\hline $6.500000000000000 \mathrm{E}+01$ & 4.900000000 \\
\hline $7.500000000000000 \mathrm{E}+01$ & $2.460000000000000 \mathrm{E}+02$ \\
\hline $8.500000000000000 \mathrm{E}+01$ & $1.180000000000000 \mathrm{E}+02$ \\
\hline $9.500000000000000 \mathrm{E}+01$ & $5.200000000000000 \mathrm{E}+01$ \\
\hline $1.050000000000000 \mathrm{E}+02$ & $4.400000000000000 \mathrm{E}+01$ \\
\hline \multicolumn{2}{|l|}{ skip 501 lines } \\
\hline \multicolumn{2}{|c|}{ Neutron Time Response for Detector \# 3} \\
\hline $5.000000000000000 \mathrm{E}+00$ & $1.548140000000000 \mathrm{E}+05$ \\
\hline $1.500000000000000 \mathrm{E}+01$ & $8.020400000000000 \mathrm{E}+04$ \\
\hline $2.500000000000000 \mathrm{E}+01$ & $1.957200000000000 \mathrm{E}+04$ \\
\hline $3.500000000000000 \mathrm{E}+01$ & $6.268000000000000 \mathrm{E}+03$ \\
\hline $4.500000000000000 \mathrm{E}+01$ & $2.429000000000000 \mathrm{E}+03$ \\
\hline $5.500000000000000 \mathrm{E}+01$ & $9.470000000000000 \mathrm{E}+02$ \\
\hline $6.500000000000000 \mathrm{E}+01$ & $4.830000000000000 \mathrm{E}+02$ \\
\hline $7.500000000000000 \mathrm{E}+01$ & $2.040000000000000 \mathrm{E}+02$ \\
\hline $8.500000000000000 \mathrm{E}+01$ & $9.800000000000000 \mathrm{E}+01$ \\
\hline $9.500000000000000 \mathrm{E}+01$ & $5.900000000000000 \mathrm{E}+01$ \\
\hline $1.050000000000000 \mathrm{E}+02$ & $3.800000000000000 \mathrm{E}+01$ \\
\hline
\end{tabular}

Figure 6.25. MCNP-DSP pulsed source output file e3a1a.1.tim. 


\subsection{FEYNMAN VARIANCE SIMULATION}

This example demonstrates the use of a Feynman variance simulation with a multi-volume, inherent spontaneous fission source. The geometry consists of a $5.0-\mathrm{cm}$ OD delta phase plutonium sphere surrounded by a delta phase plutonium shell that has 9.0-cm ID and a 15.0-cm OD. The delta phase plutonium sphere has a density of 15.48 $\mathrm{g} / \mathrm{cm}^{3}$ and is comprised of $90 \mathrm{wt} \%{ }^{239} \mathrm{Pu}, 9 \mathrm{wt} \%{ }^{240} \mathrm{Pu}$, and $1 \mathrm{wt} \%{ }^{241} \mathrm{Pu}$. Two plastic scintillation detectors are located $7.5-\mathrm{cm}$ away from the surface of the outer shell and positioned $180^{\circ}$ apart. The only source for this problem is the inherent plutonium source. The MCNP input file for this problem is provided in Fig. 6.26 and the MCNP-DSP extra data file is provided in Fig. 6.27.

\section{Description of Input File E4A1.I}

Line 1: $\quad$ The first line of the input file is the title line.

Lines 2-10: These are the cell description cards. The cells must be numbered sequentially, and cells that are not used in the model must be removed. Otherwise, MCNP-DSP will produce errors in the calculations.

Lines 12-27: These lines contain the surface description cards.

Lines 29-41: These are the data cards. The materials must be numbered sequentially. No materials can be skipped nor should unused materials be added to the input file. A separate material must be entered for each detector because the detector tallies are determined by the material in which interactions occur. The $p r d m p$ card is used to control how often data are written to the MCNP-DSP output files. The $d b c n$ card is used to change the random number seed for the MCNP-DSP because the output data for several MCNP-DSP calculations are typically combined together.

Lines 42-43: These lines are used to execute the MCNP-DSP calculations. If the first entry on the idum card is 1, the MCNP-DSP calculation is executed and other tallies are not accumulated. The files card is used to specify two files. The first file (e4a1.x) contains the extra data parameters and is opened as a sequential, formatted file on unit 21. The second file (e4a1.1) is the MCNP-DSP output file. The output file is opened as a sequential formatted file on unit 22. 


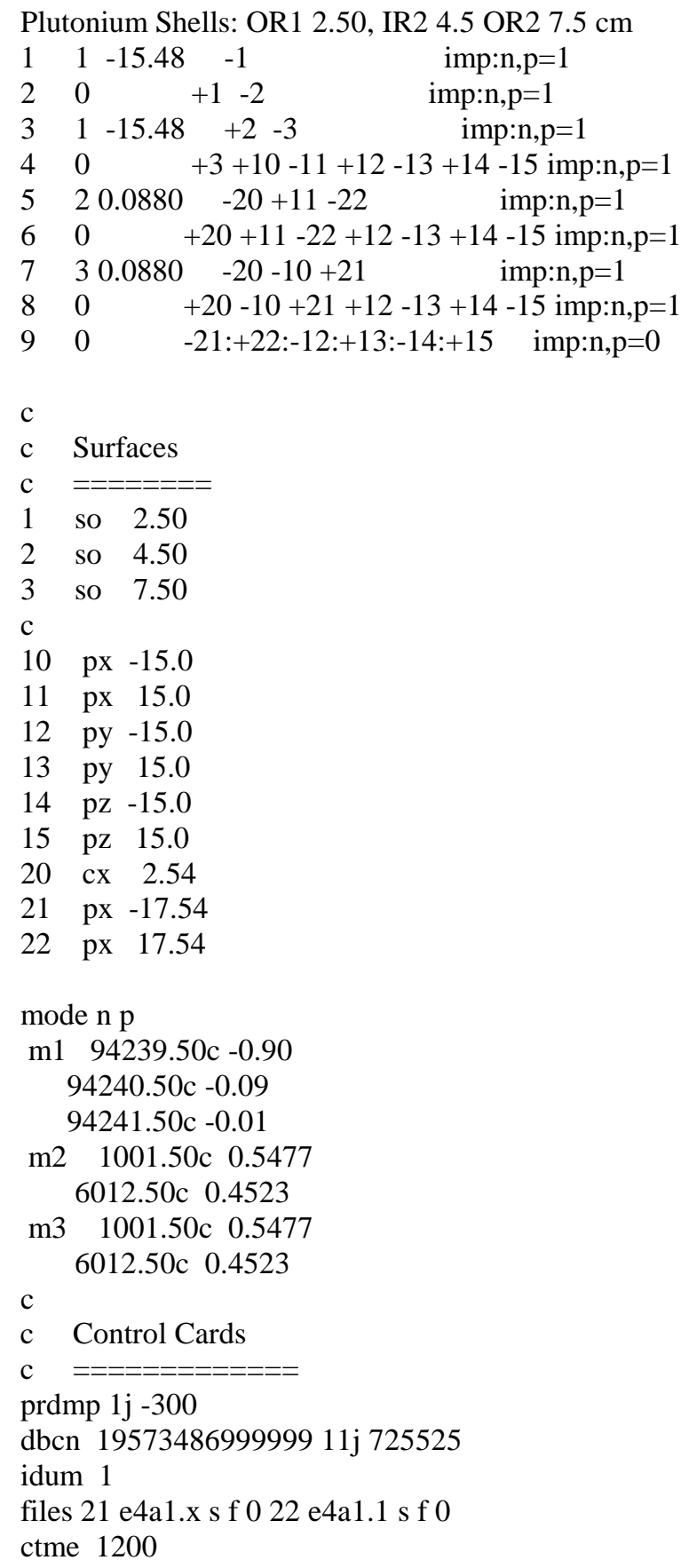

Figure 6.26. MCNP-DSP input file $e$ 4al.i. 


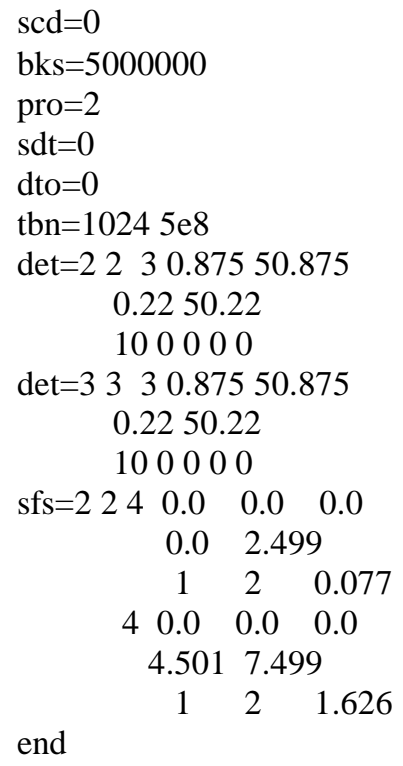

\section{Figure 6.27. MCNP-DSP input file e4a1.x.}

\section{Description of Extra Data File E1B1.X}

Line 1: $\quad$ The source disintegrations per block $(s c d)$ are zero in this example.

Line 2: $\quad$ The number of blocks to calculate $(b k s)$ is 5000000 .

Line 3: $\quad$ This specifies that this is a Feynman variance calculation (pro=2).

Line 4: $\quad$ The dead time is not invoked $(d t o=0)$.

Line 5: This line specifies the number of time channels as 1024 with a sampling rate of $500 \mathrm{MHz}$.

Lines 6-11: These lines describe the detectors in the problem. The source is detector 1 and does not need to be labeled. This problem has two detectors, 2 and 3. Both detectors are scatter detectors. Detector 2 is material 2 and type 3 while detector 3 is material 3 and type 3 . The scatter detector requires both neutron and gamma ray thresholds. The lower and upper neutron thresholds are 0.875 and $50.875 \mathrm{MeV}$ respectively. The lower and upper gamma ray thresholds are 0.22 and $50.22 \mathrm{MeV}$ respectively. The pulse generation time is set to $10 \mathrm{~ns}$. There are no energy bins in this calculation and there are no neutron or gamma ray background counts for the detector. The dead time for each detector is zero. 
Lines 12-17: These lines are used to specify the inherent spontaneous fission source for ${ }^{240} \mathrm{Pu}$. The first parameter is the total number of spontaneous fission sources. In this example, two inherent spontaneous fission sources are selected. The next entry is the number of volumes that contain inherent spontaneous fission sources. In this example, two volumes contain the inherent source. The next entry is the source volume type that is simply a spherical source. The origin of the inherent source volume is provided next. The source parameters are continued on line 13 . The first entry is the inner radius of the sphere (type 4 volume) and the next entry is the outer radius of the sphere. Note that the outer dimensions cannot correspond exactly to one of the surfaces of the problem because the source is a volume source and not a surface source. The source parameters are continued on line 14. The first entry on line 14 is the number of inherent spontaneous fission sources in this volume and is equal to one. The next entry is the spontaneous fission type (type $2={ }^{240} \mathrm{Pu}$ ). The next entry is the number of spontaneous fission per block. On line 15, the source parameters for the second volume are specified. The first entry is the volume type (type 4 volume) and is followed by the origins of the second volume. The next entries on line 16 are the inner and outer radii of the second volume. Finally, the number of inherent spontaneous fission sources for the second volume is specified along with the type and number of spontaneous fission events per block. Note that although arbitrary numbers could have been entered, the actual numbers of spontaneous fission events per block per volume have been entered. It is important to remember that the ratio of spontaneous fission events per block must be maintained.

Only a single output file is created from the Feynman variance simulation. The output summary file contains the average counts per block and the average squared counts per block for neutron, photon, and/or the combined neutron and photon detector response. The output of the summary data file can be used to estimate the Feynman variance for a particular counting interval. The user must change the sampling rate and/or the number of time bins to change the counting period because the counting period is equal to the number of time bins divided by the sampling rate. By performing several calculations with different sampling rates, the user will be able to obtain an estimate of the Feynman variance. Furthermore, if the dead time option is invoked then the affect of dead time can be investigated as long as the dead time is longer than the period of an individual time bin. 


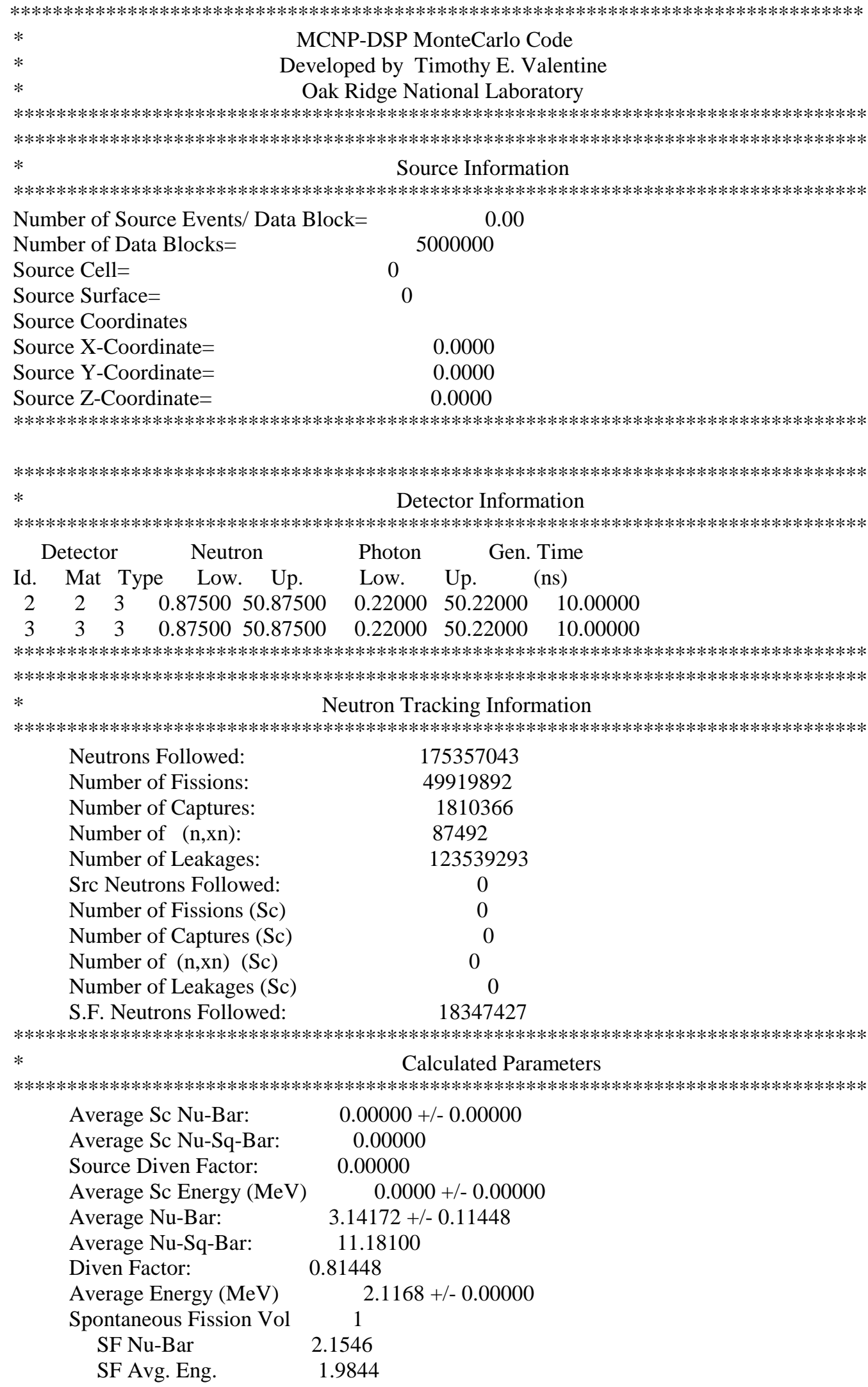

\section{Figure 6.28. MCNP-DSP output summary file e4a1.1.}




$\begin{array}{lc}\text { Spontaneous Fission Vol } & 2 \\ \text { SF Nu-Bar } & 2.1548 \\ \text { SF Avg. Eng. } & 1.9809\end{array}$

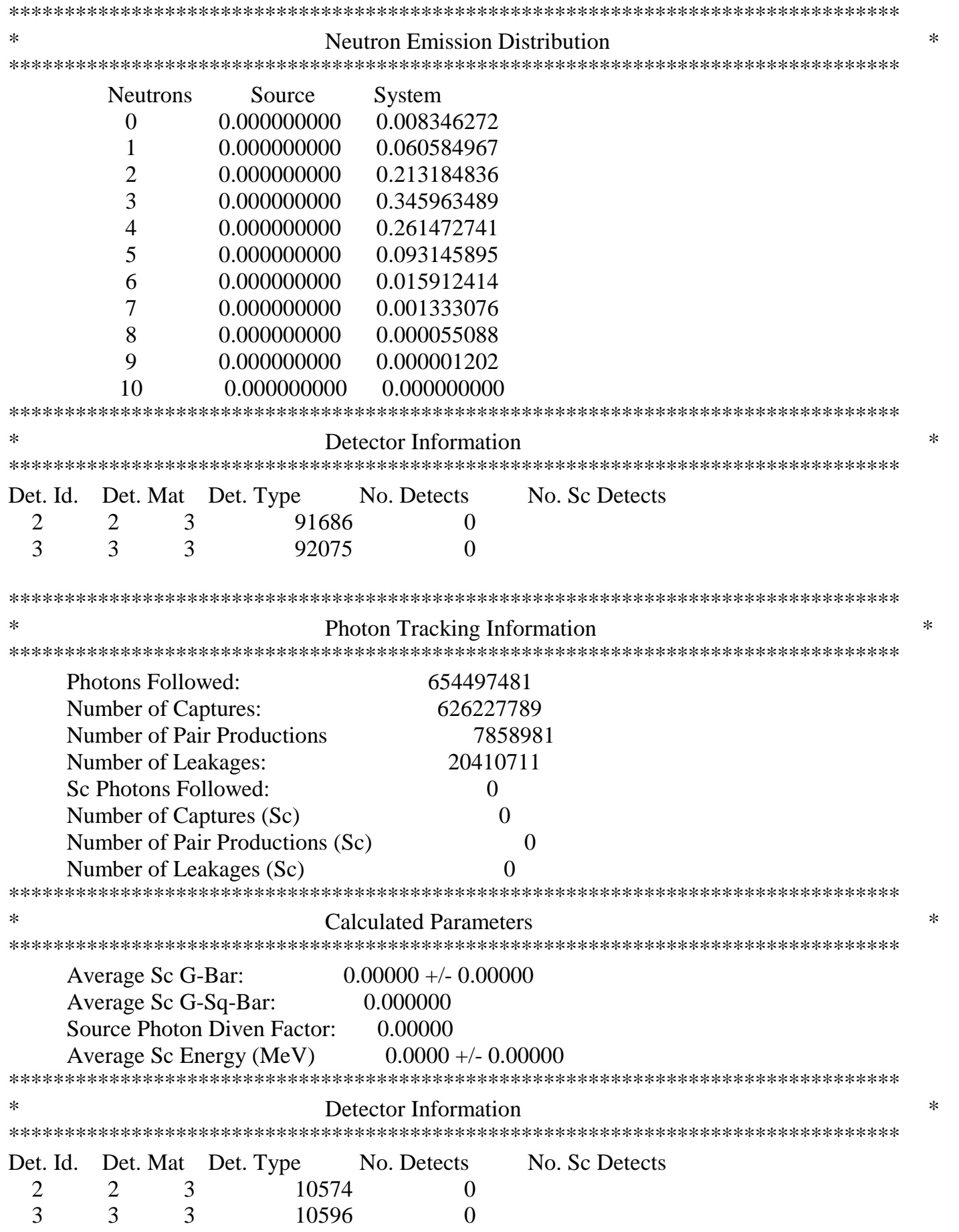

Figure 6.28. (continued). 


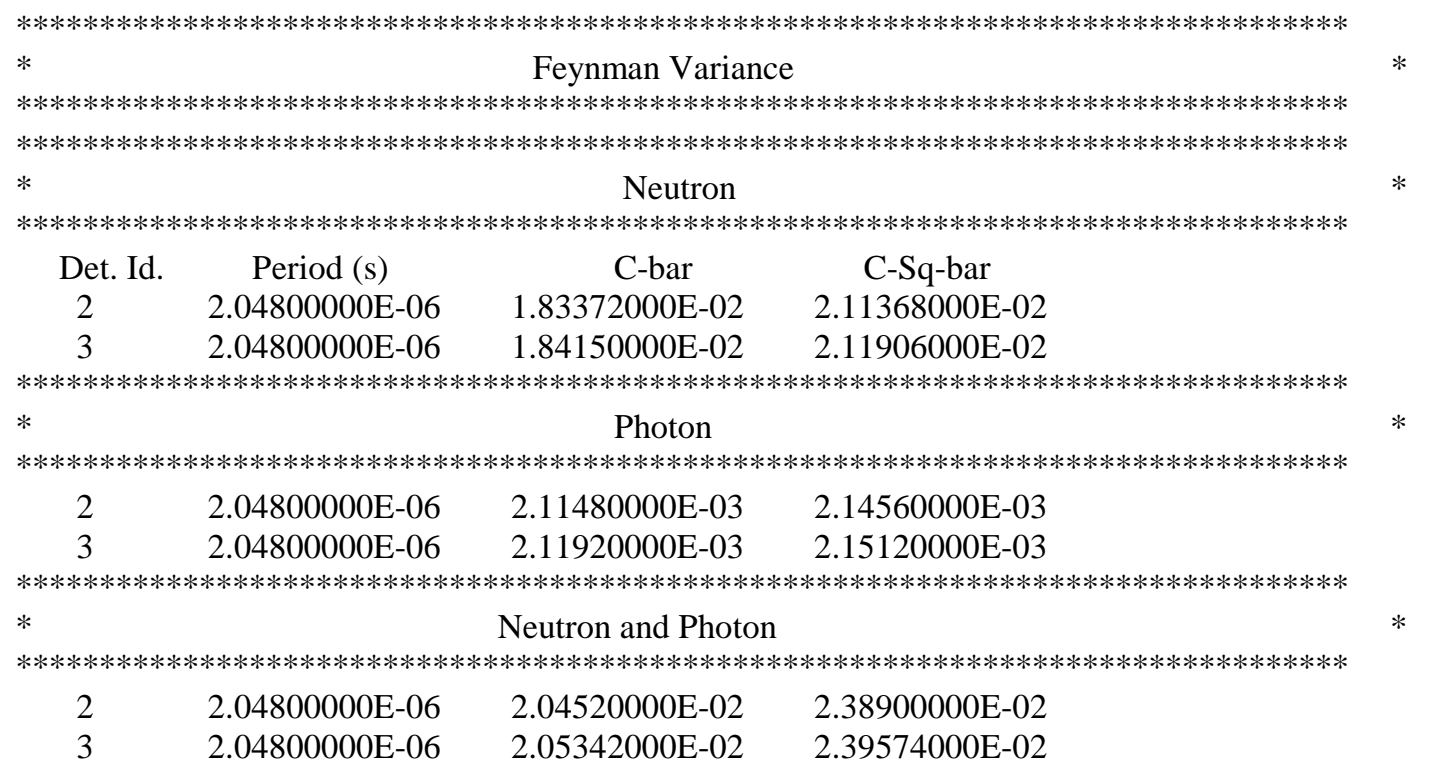

Figure 6.28. (continued).

\subsection{MULTIPLCITY SIMULATION}

This example demonstrates a passive multiplicity simulation with plutonium metal spheres. The geometry consists of a 5.0-cm OD delta phase plutonium sphere surrounded by a delta phase plutonium shell that has $9.0-\mathrm{cm}$ ID and a 15.0-cm OD. The delta phase plutonium has a density of $15.48 \mathrm{~g} / \mathrm{cm}^{3}$ and is comprised of $90 \mathrm{wt} \%{ }^{239} \mathrm{Pu}, 9 \mathrm{wt} \%{ }^{240} \mathrm{Pu}$, and $1 \mathrm{wt} \%{ }^{241} \mathrm{Pu}$. A 7.62-cm thick, 30-cm long polyethylene cylinder that contains eight ${ }^{3} \mathrm{He}$ proportional chambers that have a $2.54-\mathrm{cm}$ OD and are $30-\mathrm{cm}$ long surrounds the plutonium assembly. The detectors are modeled using the same material in this instance to demonstrate how a single response can be obtained in the multiplicity simulation that involves numerous actual detectors. The MCNP input file is given in Fig. 6.29, and the extra data file contents for the multiplicity simulation are given in Fig. 6.30. 


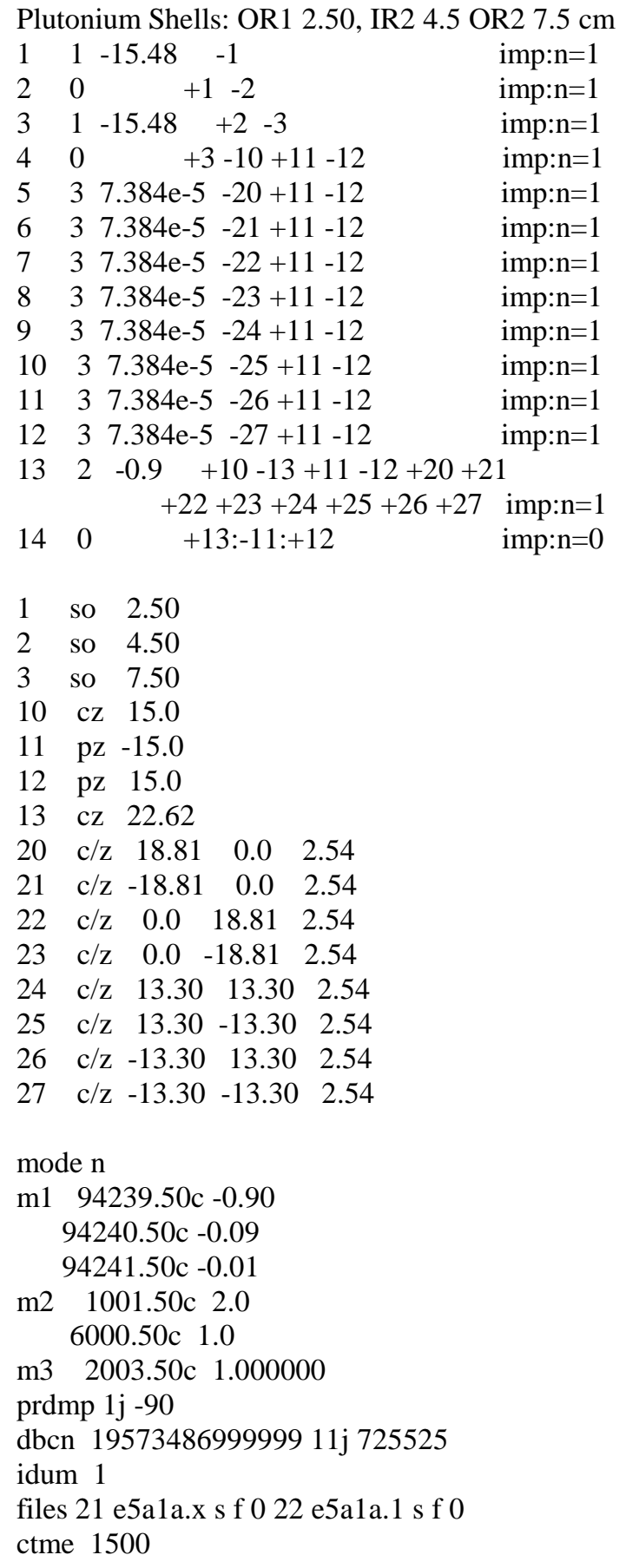

Figure 6.29. MCNP-DSP input file e5ala.i. 


\section{Description of Input File E5A1A.I}

Line 1: $\quad$ The first line of the input file is the title line.

Lines 2-16: These are the cell description cards. The cells must be numbered sequentially, and cells that are not used in the model must be removed. Otherwise, MCNP-DSP will produce errors in the calculations.

Lines 18-32: These lines contain the surface description cards.

Lines 34-42: These are the data cards. The materials must be numbered sequentially. No materials can be skipped nor should unused materials be added to the input file. The prdmp card is used to control how often data are written to the MCNP-DSP output files. The $d b c n$ card is used to change the random number seed for the MCNP-DSP because the output data for several MCNP-DSP calculations are typically combined together.

Lines 43-44: These lines are used to execute the MCNP-DSP calculations. If the first entry on the idum card is 1, the MCNP-DSP calculation is executed and other tallies are not accumulated. The files card is used to specify two files. The first file (e5a1a.x) contains the extra data parameters and is opened as a sequential, formatted file on unit 21 . The second file (e5a1a.1) is the MCNP-DSP output file. The output file is opened as a sequential formatted file on unit 22. Both files must be entered as sequential and formatted files. The record length is zero for sequential files.

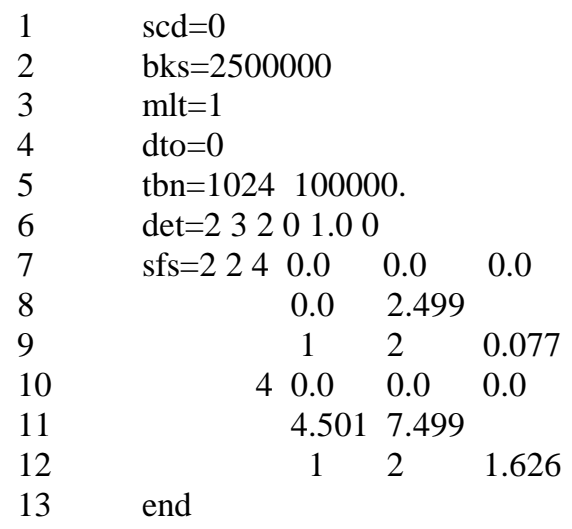

Figure 6.30. MCNP-DSP extra data file e5a1a.x. 


\section{Description of Extra Data File E5A1A.X}

Line 1: $\quad$ There are no source disintegrations per block $(s c d)$ in this example.

Line 2: $\quad$ The number of blocks to calculate $(b k s)$ is 2500000.

Line 3: $\quad$ This line specifies that this is a detector triggered multiplicity calculation.

Line 4: $\quad$ This line specifies that the dead time option be disabled.

Line 5: $\quad$ This line specifies the number of time channels as 1024 with a sampling rate of $100 \mathrm{kHz}$.

Lines 6: These lines describe the detector in the problem. The detector is a capture detector that has a $100 \%$ neutron counting efficiency.

Lines 7-12: These lines are used to specify the inherent spontaneous fission source for

${ }^{240} \mathrm{Pu}$. The first parameter is the total number of spontaneous fission sources. In this example, two inherent spontaneous fission sources are selected. The next entry is the number of volumes that contain inherent spontaneous fission sources. In this example, two volumes contain the inherent source. The next entry is the source volume type that is simply a spherical source. The origin of the inherent source volume is provided next. The source parameters are continued on line 13 . The first entry is the inner radius of the sphere (type 4 volume) and the next entry is the outer radius of the sphere. Note that the outer dimensions cannot correspond exactly to one of the surfaces of the problem because the source is a volume source and not a surface source. The source parameters are continued on line 14 . The first entry on line 14 is the number of inherent spontaneous fission sources in this volume and is equal to one. The next entry is the spontaneous fission type (type $2={ }^{240} \mathrm{Pu}$ ). The next entry is the number of spontaneous fission per block. On line 15, the source parameters for the second volume are specified. The first entry is the volume type (type 4 volume) and is followed by the origins of the second volume. The next entries on line 16 are the inner and outer radii of the second volume. Finally, the number of inherent spontaneous fission sources for the second volume is specified along with the type and number of spontaneous fission events per block. Note that although arbitrary numbers could have been entered, the actual numbers of spontaneous fission events per block per volume have been entered. It is important to remember that the ratio of spontaneous fission events per block must be maintained.

Line 13: The end parameter is used to specify the end of the extra file. This option could have also been omitted. 
Two output files are created when performing multiplicity simulations. The first contains general information on the number of counts for each detector and is specified as the second data file in the MCNP input file. The other output file contains the multiplicity signatures for the detectors. The general output filename as specified in Fig. 6.29 is e5ala.1. A second output file is written that on the Unix operating system will have the name e5ala.1.mlt. The ' $m l t$ ' indicates that the file contains multiplicity data.

\section{Description of Output Pulsed Source File E5A1A.1.MLT}

A limited amount of the multiplicity simulation output file is provided in Fig. 6.31. The output file contains the triggered and random multiplicity counts for the detector and the combined detector response. The output file has two columns that are separated by headers. The first column contains triggered multiplicity values, and the second column contains the random multiplicity values. The combined detector multiplicity values are the same as those for detector 2 because only a single detector was specified in this problem. These multiplicity values are obtained from a fictitious assembly and geometry and do not represent any actual multiplicity measurement. 


\begin{tabular}{|c|c|c|}
\hline \multicolumn{3}{|c|}{ Detector Triggered Multiplicity } \\
\hline \multicolumn{3}{|c|}{ Neutron } \\
\hline \multicolumn{3}{|c|}{ Detector \# 2} \\
\hline Value & Triggered & Random \\
\hline 0 & 989774 & 1496014 \\
\hline 1 & 582268 & 418522 \\
\hline 2 & 399462 & 185323 \\
\hline 3 & 297262 & 103156 \\
\hline 4 & 230894 & 66894 \\
\hline 5 & 184042 & 47092 \\
\hline 6 & 149142 & 35046 \\
\hline 7 & 122115 & 27060 \\
\hline 8 & 100925 & 21195 \\
\hline 9 & 83324 & 17550 \\
\hline 10 & 69274 & 14024 \\
\hline \multicolumn{3}{|c|}{ skip 1013 lines } \\
\hline \multirow{2}{*}{\multicolumn{3}{|c|}{$\begin{array}{lcc} & & \\
1024 & 0 & 0 \\
* * * * * * * * * * * * * * * * * * * * * * * * * * * * * * * * * * * * * * *\end{array}$}} \\
\hline & & \\
\hline \multicolumn{3}{|c|}{$\begin{array}{l}* \text { Combined Detector Distributions } \\
* * * * * * * * * * * * * * * * * * * * * * * * * * * * * * * * * * * * * * *\end{array}$} \\
\hline \multicolumn{3}{|c|}{ Detector Triggered Multiplicity } \\
\hline \multicolumn{3}{|c|}{ Neutron } \\
\hline Value & Triggered & Random \\
\hline 0 & 989774 & 1496014 \\
\hline 1 & 582268 & 418522 \\
\hline 2 & 399462 & 185323 \\
\hline 3 & 297262 & 103156 \\
\hline 4 & 230894 & 66894 \\
\hline 5 & 184042 & 47092 \\
\hline 6 & 149142 & 35046 \\
\hline 7 & 122115 & 27060 \\
\hline 8 & 100925 & 21195 \\
\hline 9 & 83324 & 17550 \\
\hline 10 & 69274 & 14024 \\
\hline
\end{tabular}

Figure 6.31. MCNP-DSP multiplicity output file e5a1a.1.mlt. 
APPENDIX A

MCNP-DSP INPUT AND OUTPUT FILES 


\title{
APPENDIX A MCNP-DSP INPUT AND OUTPUT FILES
}

MCNP-DSP is executed in the same manner as standard MCNP. To perform a MCNP-DSP simulation, the user must create a standard MCNP input file and construct an extra data file for MCNP-DSP. The extra data file contains the various options for specifying the source type, detector type, and processing mode for the simulation. A typical execution command line for executing MCNP-DSP is as follows:

$$
\text { mcnp.dsp inp=file.i outp=file.o }
$$

In this example, "file. $i$ " is the standard MCNP input file and "file.o" is the standard MCNP output file. To perform a MCNP-DSP simulation, the input file "file. $i$ " must contain the following data cards:

\author{
idum 1 \\ files 21 file.x s f 022 file. 1 s fO
}

The data card idum is used to instruct the program to perform the MCNP-DSP simulations. If this data card is omitted, MCNP-DSP will perform a standard MCNP calculation assuming that the user specifies the source and tally options appropriately. In this example, the extra data file is "file.x" and is opened on unit 21 as a sequential formatted data file with a record length of zero. The extra data file must always be an ASCII file. A second file is also specified with the files data card. This second file is the MCNP-DSP output summary file. This file contains a summary of the neutron and/or gamma ray tracking information and detection information. Additional output files are also created in addition to the MCNP-DSP summary file. These output files depend on the simulation type.

For frequency analysis simulations, the additional output files contain the frequency spectra between the source and two detectors. The names of the frequency spectra output files are created from the MCNP-DSP output summary file. The name of the frequency spectra output files depends on the operating system. For UNIX operating systems, the frequency spectra files would have the names file.1.123.fft.n, file.1.123.fft.p, and file.1.123.fft.np assuming that the simulation was a dual neutron and photon problem. The first file contains the spectra for the neutron response, the second file contains the spectra for the gamma response, and the third file contains the spectra for the combined neutron and gamma ray response as indicated by the last extension. The other filename components have distinct meanings. The first part that has the ' 123 ' indicates that this file contains the frequency spectra for the auto spectra of detectors \#1, \#2, and \#3, and the cross spectra between 1 and 2,1 and 3 , and 2 and 3 . The ' $f f t$ ' indicates that the file contains data that have been Fourier transformed. If the simulation has more than two detectors, then frequency spectra files will be created for each pair of combinations of detectors. For example if the simulation contains a source and three detectors additional frequency spectra files will be generated as file.1.124.fft.n, file.1.124.fft.p, file.1.124.fft.np, file.1.134.fft.n, file.1.134.fft.p, and file.1.134.fft.np. The files with '124' 
would contain the frequency spectra for the auto spectra of detectors \#1, \#2, and \#4, and the cross spectra between 1 and 2, 1 and 4, and 2 and 4. Likewise, the files with ' 134 ' would contain the frequency spectra for the auto spectra of detectors $\# 1$, \#3, and \#4, and the cross spectra between 1 and 3,1 and 4, and 3and 4. For the PC operating system, the frequency spectra files have different names because of limitations with DOS filenames. The frequency spectra files would have names file-1.fn1, file-1.fpl, and file-1.fcl for a simulation with a source and two detectors. The ' $f n l$ ' file contains the frequency spectra involving the neutron response for the source and both detectors, the ' $f p l$ ' file contains the frequency spectra involving the photon response for the source and both detectors, and the ' $f c l$ ' file contains the frequency spectra involving the neutron and photon response for the source and both detectors. If the simulation contains a source and three detectors additional frequency spectra files will be generated as file-1.fn2, file-1.fp2, file1.fc2, file-1.fn3, file-1.fp3, and file-1.fc3.

For correlation analysis simulations, the additional output files contain the correlation functions between the source and two detectors. The names of the correlation output files are created from the MCNP-DSP output summary file. The name of the correlation output files depends on the operating system. For UNIX operating systems, the correlation files would have the names file.1.123.cor.n, file.1.123.cor.p, and file.1.123.cor.np assuming that the simulation was a dual neutron and photon problem. The first file contains the correlation functions for the neutron response, the second file contains the correlation functions for the gamma response, and the third file contains the correlation functions for the combined neutron and gamma ray response as indicated by the last extension. The other filename components have distinct meanings. The first part that has the ' 123 ' indicates that this file contains the frequency spectra for the auto correlation function of detectors $\# 1, \# 2$, and $\# 3$, and the cross correlation functions between 1 and 2, 1 and 3 , and 2 and 3. The 'cor' indicates that the file contains correlation data. If the simulation has more than two detectors, then correlation files will be created for each pair of combinations of detectors. For example if the simulation contains a source and three detectors additional correlation files will be generated as file.1.124.cor.n, file.1.124.cor.p, file.1.124.cor.np, file.1.134.cor.n, file.1.134.cor.p, and file.1.134.cor.np. The files with '124' would contain the auto correlation functions of detectors \#1,\#2, and \#4, and the cross correlation functions between 1 and 2, 1 and 4, and 2 and 4. Likewise, the files with '134' would contain the auto correlation functions of detectors \#1, \#3, and \#4, and the cross correlation functions between 1 and 3,1 and 4, and 3and 4. For the PC operating system, the correlation files have different names because of limitations with DOS filenames. The correlation files would have names file-1.cn1, file1.cpl, and file-1.ccl for a simulation with a source and two detectors. The 'cnl' file contains the correlation functions involving the neutron response for the source and both detectors, the ' $c p l$ ' file contains the correlation functions involving the photon response for the source and both detectors, and the ' $c c l$ ' file contains the correlation functions involving the neutron and photon response for the source and both detectors. If the simulation contains a source and three detectors additional correlation files will be generated as file-1.cn2, file-1.cp2, file-1.cc2, file-1.cn3, file-1.cp3, and file-1.cc3. 
For pulsed neutron simulations, only one additional output file is written. For the UNIX operating systems, the pulsed source file would be file.1.tim. This file contains the counts after a source event for the neutron, photon, and combined neutron and photon response of the detector. For the PC operating system, the file name would be file-1.tim. The extension 'tim' refers to time domain.

For multiplicity simulations, only one additional output file is written. For UNIX operating systems, the multiplicity file would be file.1.mlt. This file contains the detector or source triggered multiplicity values for the neutron, photon, and combined neutron and photon response of the detector. For the PC operating system, the file name would be file1.mlt. The extension ' $m l t$ ' refers to multiplicity.

For the Feynman variance simulation, no additional output files are written. The average counts and average squared counts per block are written to the MCNP-DSP output summary file. 
APPENDIX B

SPONTANEOUS FISSION NEUTRON DISTRIBUTIONS 


\section{APPENDIX B. SPONTANEOUS FISSION NEUTRON DISTRIBUTIONS}

The $\mathrm{P}(v)$ data for ${ }^{252} \mathrm{Cf}$ was obtained from Spencer's measured data and are listed in Table B.1. The $\mathrm{P}(v)$ data for ${ }^{238} \mathrm{U},{ }^{240} \mathrm{Pu},{ }^{242} \mathrm{Pu},{ }^{242} \mathrm{Cm}$, and ${ }^{244} \mathrm{Cm}$ were obtained from Zucker and Holden's tabulated data and are listed in Tables B.2 to B.6.

Table B.1. $\mathbf{P}(v)$ probabilities for ${ }^{252} \mathrm{Cf}$

\begin{tabular}{|c|c|}
\hline$v$ & $\mathrm{P}(\mathrm{v})$ \\
0 & 0.00211 \\
\hline 1 & 0.02467 \\
\hline 2 & 0.12290 \\
\hline 3 & 0.27144 \\
\hline 4 & 0.30763 \\
\hline 5 & 0.18770 \\
\hline 6 & 0.06770 \\
\hline 7 & 0.01406 \\
\hline 8 & 0.00167 \\
\hline 9 & 0.00010 \\
\hline
\end{tabular}

Table B.2. $P(v)$ probabilities for ${ }^{238} U$

\begin{tabular}{|c|c|}
\hline$v$ & $\mathrm{P}(v)$ \\
\hline 0 & 0.0481677 \\
\hline 1 & 0.2485215 \\
\hline 2 & 0.4253044 \\
\hline 3 & 0.2284094 \\
\hline 4 & 0.0423438 \\
\hline 5 & 0.0072533 \\
\hline
\end{tabular}

Table B.3. $\mathrm{P}(v)$ probabilities for ${ }^{240} \mathrm{Pu}$

\begin{tabular}{|c|c|}
\hline$v$ & $\mathrm{P}(\mathrm{v})$ \\
\hline 0 & 0.0631852 \\
\hline 1 & 0.2319644 \\
\hline 2 & 0.3333230 \\
\hline 3 & 0.2528207 \\
\hline 4 & 0.0986461 \\
\hline 5 & 0.0180199 \\
\hline 6 & 0.0020406 \\
\hline
\end{tabular}


Table B.4. $P(v)$ probabilities for ${ }^{242} \mathrm{Pu}$

\begin{tabular}{|c|c|}
\hline$v$ & $\mathrm{P}(\mathrm{v})$ \\
\hline 0 & 0.0679423 \\
\hline 1 & 0.2293159 \\
\hline 2 & 0.3341228 \\
\hline 3 & 0.2475507 \\
\hline 4 & 0.0996922 \\
\hline 5 & 0.0182398 \\
\hline 6 & 0.0031364 \\
\hline
\end{tabular}

Table B.5. $\mathrm{P}(v)$ probabilities for ${ }^{242} \mathrm{Cm}$

\begin{tabular}{|c|c|}
\hline$v$ & $\mathrm{P}(\mathrm{v})$ \\
\hline 0 & 0.0212550 \\
\hline 1 & 0.1467407 \\
\hline 2 & 0.3267531 \\
\hline 3 & 0.3268277 \\
\hline 4 & 0.1375090 \\
\hline 5 & 0.0373815 \\
\hline 6 & 0.0025912 \\
\hline 7 & 0.0007551 \\
\hline 8 & 0.0001867 \\
\hline
\end{tabular}

Table B.6. $\mathbf{P}(v)$ probabilities for ${ }^{244} \mathrm{Cm}$

\begin{tabular}{|c|c|}
\hline$v$ & $\mathrm{P}(\mathrm{v})$ \\
\hline 0 & 0.0150050 \\
\hline 1 & 0.1161725 \\
\hline 2 & 0.2998427 \\
\hline 3 & 0.3331614 \\
\hline 4 & 0.1837748 \\
\hline 5 & 0.0429780 \\
\hline 6 & 0.0087914 \\
\hline 7 & 0.0002744 \\
\hline
\end{tabular}


APPENDIX C

SPONTANEOUS FISSION NEUTRON ENERGY SPECTRA 


\section{APPENDIX C. SPONTANEOUS FISSION NEUTRON ENERGY SPECTRA}

The energy spectra of neutrons from spontaneous fission can be represented with a Maxwellian or a Watt spectrum. The prompt neutron energy spectrum, $N(E)$, represented by the Maxwellian spectrum is

$$
N(E)=\frac{2}{\sqrt{\pi}} \frac{\sqrt{E}}{T^{3 / 2}} e^{-E / T},
$$

where $E$ is the energy on the neutron and $T$ is the nuclear temperature. The nuclear temperature is typically $1.42 \mathrm{MeV}$ for ${ }^{252} \mathrm{Cf}$. The Maxwellian does not agree precisely with the measured spectrum for ${ }^{252} \mathrm{Cf}$; therefore, Mannhart ${ }^{17}$ developed an energy dependent correction factor, $R(E)$, which when multiplied by the Maxwellian spectrum would reproduce the measured spectrum. A least-squares polynomial regression model was used to obtain a functional form for Mannhart's correction factor. For energies less than $5 \mathrm{MeV}$, the correction factor is represented by

$$
\begin{aligned}
& R(E)=0.955+0.0707 E-0.0444 E^{2}+0.01998 E^{3} \\
& -0.00457 E^{4}+0.00036 E^{5} .
\end{aligned}
$$

For energies greater than $5 \mathrm{MeV}$, the correction factor is represented as

$$
R(E)=1.16-0.0432 E+0.00185 E^{2}-0.0000316 E^{3}
$$

The energy ranges were chosen such that the functional representation adequately reproduces Mannhart's discrete points. Other fits could be applied to Mannhart's correction factor that when multiplied by the Maxwellian should adequately reproduce the measured spectra. The average neutron energy obtained from the corrected spectrum is $2.13 \mathrm{MeV}$, which agrees well with the measured value. The integral of the corrected spectrum as a function of energy was normalized to unity. A least-squares polynomial regression was used to fit the energy as a function of the normalized integral, that is, making the energy a polynomial function of the random variable.

The energy spectra of the inherent spontaneous fission sources is represented by the Watt spectrum,

$$
N(E)=C e^{-E / a} \sinh (b E)^{1 / 2},
$$

where $E$ is the neutron energy, $C$ is a normalization constant, and $a$ and $b$ are the parameters for the Watt spectrum. Some of the Watt parameters for the inherent spontaneous fission sources can be found in the MCNP manual, and others were supplied by David Madland of Los Alamos National Laboratory. A listing of the Watt parameters for the inherent spontaneous fission sources is presented in Table C.1. 
Table C.1. Watt parameters for inherent spontaneous fission sources

\begin{tabular}{|c|c|c|}
\hline Isotope & $\mathrm{a}(\mathrm{MeV})$ & $\mathrm{b}\left(\mathrm{MeV}^{-1}\right)$ \\
\hline${ }^{238} \mathrm{U}$ & 0.78 & 5.194 \\
\hline${ }^{240} \mathrm{Pu}$ & 0.799 & 4.903 \\
\hline${ }^{242} \mathrm{Pu}$ & 0.834 & 4.432 \\
\hline${ }^{242} \mathrm{Cm}$ & 0.891 & 4.046 \\
\hline${ }^{244} \mathrm{Cm}$ & 0.906 & 3.848 \\
\hline
\end{tabular}


APPENDIX D

SPONTANEOUS FISSION GAMMA RAY DISTRIBUTIONS 


\section{APPENDIX D. SPONTANEOUS FISSION GAMMA RAY DISTRIBUTIONS}

The $\mathrm{P}(v)$ data for ${ }^{252} \mathrm{Cf}$ was obtained from Brunson's measured data and are listed in Table D.1. The parameters used to create the probability distributions for ${ }^{238} \mathrm{U},{ }^{240} \mathrm{Pu}$, ${ }^{242} \mathrm{Pu},{ }^{242} \mathrm{Cm}$, and ${ }^{244} \mathrm{Cm}$ are provided in Table D.2.

Table D.1. $\mathrm{P}(\mathrm{g})$ probabilities for ${ }^{252} \mathrm{Cf}$

\begin{tabular}{|c|c|}
\hline $\begin{array}{l}\mathrm{g} \\
0 \\
\end{array}$ & $\begin{array}{c}\mathrm{P}(\mathrm{g}) \\
5.162699 \mathrm{e}-04\end{array}$ \\
\hline 1 & $3.742057 \mathrm{e}-03$ \\
\hline 2 & $1.360482 \mathrm{e}-02$ \\
\hline 3 & $3.312786 \mathrm{e}-02$ \\
\hline 4 & $6.090540 \mathrm{e}-02$ \\
\hline 5 & $9.043537 \mathrm{e}-02$ \\
\hline 6 & $1.133984 \mathrm{e}-01$ \\
\hline 7 & $1.240985 \mathrm{e}-01$ \\
\hline 8 & $1.216759 \mathrm{e}-01$ \\
\hline 9 & $1.092255 \mathrm{e}-01$ \\
\hline 10 & $9.137106 \mathrm{e}-02$ \\
\hline 11 & $7.219960 \mathrm{e}-02$ \\
\hline 12 & $5.438050 \mathrm{e}-02$ \\
\hline 13 & $3.923091 \mathrm{e}-02$ \\
\hline 14 & $2.714690 \mathrm{e}-02$ \\
\hline 15 & $1.800781 \mathrm{e}-02$ \\
\hline 16 & $1.143520 \mathrm{e}-02$ \\
\hline 17 & $6.942099 \mathrm{e}-03$ \\
\hline 18 & $4.025720 \mathrm{e}-03$ \\
\hline 19 & $2.229510 \mathrm{e}-03$ \\
\hline 20 & $1.179602 \mathrm{e}-03$ \\
\hline 21 & $5.966936 \mathrm{e}-04$ \\
\hline 22 & $2.888766 \mathrm{e}-04$ \\
\hline 23 & $1.340137 \mathrm{e}-04$ \\
\hline 24 & $5.965291 \mathrm{e}-05$ \\
\hline 25 & $2.551191 \mathrm{e}-05$ \\
\hline 26 & $1.049692 \mathrm{e}-05$ \\
\hline 27 & $4.160575 \mathrm{e}-06$ \\
\hline 28 & $1.590596 \mathrm{e}-06$ \\
\hline
\end{tabular}


Table D.2. Parameters of gamma rays from spontaneous fission of ${ }^{238} \mathrm{U},{ }^{238} \mathrm{Pu},{ }^{240} \mathrm{Pu},{ }^{242} \mathrm{Pu},{ }^{242} \mathrm{Cm}$, and ${ }^{244} \mathrm{Cm}$

\begin{tabular}{|c|c|c|c|}
\hline Isotope & $\begin{array}{c}\text { Total energy } \\
(\mathrm{MeV})\end{array}$ & Average number & $\begin{array}{c}\text { Average energy } \\
(\mathrm{MeV})\end{array}$ \\
\hline${ }^{238} \mathrm{U}$ & $6.06 \pm 0.03$ & $6.36 \pm 0.47$ & $0.95 \pm 0.07$ \\
\hline${ }^{238} \mathrm{Pu}$ & $6.14 \pm 0.03$ & $6.34 \pm 0.46$ & $0.97 \pm 0.07$ \\
\hline${ }^{240} \mathrm{Pu}$ & $6.07 \pm 0.03$ & $6.40 \pm 0.47$ & $0.95 \pm 0.07$ \\
\hline${ }^{242} \mathrm{Pu}$ & $6.05 \pm 0.03$ & $6.51 \pm 0.49$ & $0.93 \pm 0.07$ \\
\hline${ }^{242} \mathrm{Cm}$ & $6.26 \pm 0.04$ & $6.62 \pm 0.49$ & $0.95 \pm 0.07$ \\
\hline${ }^{244} \mathrm{Cm}$ & $6.40 \pm 0.04$ & $6.90 \pm 0.52$ & $0.93 \pm 0.07$ \\
\hline
\end{tabular}

These parameters are used with the negative binomial distribution to determine the probability distribution functions for spontaneous fission gamma rays. The negative binomial distribution is defined as

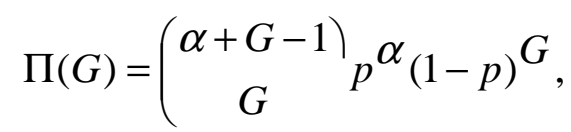

with distribution parameters defined as $p=\bar{x} / \sigma^{2}$ and $\alpha=p \bar{x} /(1-p)$. The parameter $\alpha$ can be redefined in terms of the relative width of the distribution as

$$
\alpha=\left(D_{\gamma}-1\right)^{-1}
$$

Likewise, the parameter $p$ can be redefined as

$$
p=\frac{\alpha}{\alpha+\bar{G}}
$$

The value for $\alpha$ is assumed to be a constant and equal to approximately 26 and $\bar{G}$ is the average number of gamma rays from spontaneous fission. A detailed description of this representation of prompt fission gamma rays is provided in ref. 30 . 
This page intentionally left blank. 


\section{REFERENCES}

1. T. E. Valentine, MCNP-DSP: A Neutron and Gamma Ray Monte Carlo Calculation of Source-Driven Noise-Measured Parameters, Ph.D. Dissertation, University of Tennessee, Knoxville, 1995.

2. T. E. Valentine and J. T. Mihalczo, "MCNP-DSP: A Neutron and Gamma Ray Monte Carlo Calculation of Source-Driven Noise-Measured Parameters," Ann. Nucl. Eng. 23 161271 (1996).

3. J. F. Briesmeister, ed., MCNP4A-A General Monte Carlo N-Particle Transport Code, La-12625-M, Los Alamos National Laboratory, 1993.

4. V. K. Paré and J. T Mihalczo, "Reactivity from Power Spectral Density Measurements with Californium-252," Nucl. Sci. Eng. 56, 213 (1975).

5. E. P. Ficaro, KENO-NR: A Monte Carlo Code Simulating the ${ }^{252}$ Cf-Source-Driven Noise Analysis Experimental Method for Determining Subcriticality, Ph.D. Dissertation, University of Michigan, 1991.

6. L. M. Petrie, and N. F. Landers, "KENO V.a: An Improved Monte Carlo Criticality Program with Supergrouping," ORNL/NUREG/CSD-2, Oak Ridge National Laboratory, 1984.

7. J. D. Orndoff, "Prompt Neutron Periods of Metal Critical Assemblies," Nucl. Sci. Eng., 2, 450 (1957).

8. B. E. Simmons, and J. S. King, "A Pulsed Neutron Technique for Reactivity Determination," Nucl. Sci. Eng., 3, 595 (1958).

9. C. W. Ricker, D. N. Fry, E. R. Mann, and S. H. Hanauer, "Investigation of Negative Reactivity Measurement by Neutron Fluctuation Analysis," Proc. of the Symposium on Noise Analysis in Nuclear Systems, University of Florida, Gainesville, 1963.

10. M. S. Krick and J. E. Swansen, "Neutron Multiplicity and Multiplication Measurements," Nucl. Instrum. Methods, 219, 384-393 (1984).

11. R. P. Feynman, F. DeHoffman, and R. Serber, "Dispersion of the Neutron Emission in U-235 Fission,” J. Nucl. Energy, 3, 64 (1956).

12. J. Terrel, Phys. Rev. 113, 527 (1959). 
13. M. S. Zucker, and N. E. Holden, "Energy Dependence of Neutron Multiplicity $\mathrm{P}_{v}$ in Fast-Neutron-Induced Fission for ${ }^{235,238} \mathrm{U}$ and ${ }^{239} \mathrm{Pu}$," BNL-38491, 1986.

14. R. Gwin, R. R. Spencer, and R. W. Ingle, "Measurements of the Energy Dependence of Prompt Neutron Emission from ${ }^{233} \mathrm{U},{ }^{235} \mathrm{U},{ }^{239} \mathrm{Pu}$, and ${ }^{241} \mathrm{Pu}$ for $\mathrm{E}_{\mathrm{n}}=0.005$ to $10 \mathrm{eV}$ Relative to Emission from Spontaneous Fission of ${ }^{252} \mathrm{Cf}$," Nucl. Sci. Eng., 87, 381 (1984).

15. J. Frehaut, "Neutron Multiplicity Distribution in Fast Neutron-Induced Fission," Proc. of IAEA Consultant's Meeting on Physics of Neutron Emission in Fission, Mito, Japan (1988).

16. R. R. Spencer, R. Gwin, R. and Ingle, "A Measurement of the Average Number of Prompt Neutrons from Spontaneous Fission of Californium-252," Nucl. Sci. Eng. 80, 603 (1982).

17. J. W. Boldeman and M. G. Hines, "Prompt Neutron Emission Probabilities Following Spontaneous and Thermal Neutron Fission," Nucl. Sci. Eng., 91, 114 (1985).

18. N. E. Holden and M. S. Zucker, "A Reevaluation of the Average Prompt Neutron Emission Multiplicity (Nubar) Values from Fission of Uranium and Transuranium Nuclides," BNL-NCS-35513, Brookhaven National Laboratory.

19. C. Budtz-Jorgensen and H. H. Knitter, "Simultaneous Investigation of Fission Fragments and Neutrons in ${ }^{252} \mathrm{Cf}$ (SF)," Nucl. Phy. A490, 307 (1988).

20. J. R. Nix, Los Alamos National Laboratory, personal communication 1994.

21. W. Mannhart, "Evaluation of the Cf-252 Fission Neutron Spectrum Between 0 $\mathrm{MeV}$ and $20 \mathrm{MeV}$," Proc. Advisory Group Mtg. Neutron Sources, Leningrad, USSR, 1986 (IAEA-TECDOC-410), Vienna, 1987.

22. D. G. Madland and J. R. Nix, "Prompt Fission Neutron Spectra and Average Prompt Neutron Multiplicities," NEANDC Specialist's Meeting on Yields and Decay Data of Fission Products, Brookhaven National Laboratory, BNL 51778 (1984).

23. F. H. Frohner, "Evaluation of ${ }^{252} \mathrm{Cf}$ Prompt Fission Neutron Data from 0 to $20 \mathrm{MeV}$ by Watt Spectrum Fit," Nucl. Sci. Eng., 106, 345 (1990).

24. R. Seamon, Los Alamos National Laboratory, personal communication 1994.

25. R. Vandenbosch and J. R. Huizenga, Nuclear Fission, Academic Press, New York, 1973. 
26. G. S. Brunson, Jr., Multiplicity and Correlated Energy of Gamma Rays Emitted in the Spontaneous Fission of Californium-252, Ph.D. Thesis, Univ. of Utah, 1982.

27. C. Wagemans, The Nuclear Fission Process, CRC Press, Inc., Boca Raton, Florida, 1991.

28. F. C. Maienschein, R. W. Peelle, T. A. and Love, Neutron Phys. Ann. Prog. Rep. For Sept. 1, 1958, ORNL-2609, Oak Ridge National Laboratory.

29. H. Goldstein, Fundamental Aspects of Reactor Shielding, Addison-Wesley Publishing Company, Inc., Reading, Massachusetts, 1959.

30. T. E. Valentine, "Evaluation of Prompt Fission Gamma Rays for Simulating Nuclear Safeguard Measurements," ORNL/TM-1999/300, Oak Ridge National Laboratory (1999).

31. H. Nifenecker, C. Signarbieux, M. Ribrag, J. Poitou, and J. Matuszek, "GammaNeutron Competition in the De-excitation Mechanism of the Fission Fragments of ${ }^{252}$ Cf," Nucl. Phys. A189, 209 (1972).

32. A. V. Oppenheim and R. W. Schafer, Discrete-Time Signal Processing, PrenticeHall Inc., Englewood Cliffs, New Jersey, 1989. 


\section{INTERNAL DISTRIBUTION}

1. Felix C. Difilippo

2. Jess C. Gehin

3. Calvin M. Hopper

4. Bernadette Lugue Kirk

5. Michael A. Kuliasha

6. Luiz C. Leal

7. Cecil V. Parks
8. John Paul Renier

9. Royce O. Sayer

10-14. Timothy E. Valentine

15. Robert Michael Westfall

16-20. RSICC

21. Laboratory Records

22-23. Laboratory Records (OSTI)

\section{EXTERNAL DISTRIBUTION}

24. Mark E. Abhold, Los Alamos National Laboratory, P.O. Box 1663 NIS-5 MS E540, Los Alamos, NM 87545.

25. Steven E. Aumeier, Argonne National Laboratory, P.O. Box 2528, Idaho Falls, ID 83403.

26. D. E. Carlson, U.S. Nuclear Regulatory Commission, Reactor and Plant System Branch, Division of System Research, Office of Nuclear Regulatory Research, MS T-10 G6, RM T-10, 17 , Washington, DC 20555-0001.

27. Jerry Cole, Idaho National Engineering Lab, P.O. Box 1625 MS-2114, Idaho Falls ID 83415.

28. R. A. Forster, Los Alamos National Laboratory, P.O. Box 1663 X-TM MS B226, Los Alamos, NM 87545.

29. Larry B. Foulke, Westinghouse Electric Corp. Bettis Atomic Power Laboratory, P.O. Box 79, West Mifflin, PA 15122-0079.

30. Victor Gavron, Los Alamos National Laboratory, P.O. Box 1663 NIS-5 MS-E540, Los Alamos, NM 87545.

31. Thomas B. Gosnell, Lawrence Livermore National Laboratory, 7000 East Avenue L-175, Livermore, CA 94550.

32. James F. Morgan, Lawrence Livermore National Laboratory, 7000 East Avenue L-175, Livermore, CA 94550.

33. M. M. Pickrell, Los Alamos National Laboratory, P.O. Box 1663 NIS-5 MS-E540, Los Alamos, NM 87545. 